\title{
Symmetric Willmore surfaces of revolution satisfying arbitrary Dirichlet boundary data*
}

\author{
Anna Dall'Acqua, Steffen Fröhlich, Hans-Christoph Grunau, Friedhelm Schieweck
}

December 16, 2008

Dedicated to Prof. E. Heinz on the occasion of his 85th birthday.

\begin{abstract}
We consider the Willmore boundary value problem for surfaces of revolution where, as Dirichlet boundary conditions, any symmetric set of position and angle may be prescribed. Using direct methods of the calculus of variations, we prove existence and regularity of minimising solutions. Moreover, we estimate the optimal Willmore energy and prove a number of qualitative properties of these solutions. Besides convexity-related properties we study in particular the limit when the radii of the boundary circles converge to 0 , while the "length" of the surfaces of revolution is kept fixed. This singular limit is shown to be the sphere, irrespective of the prescribed boundary angles.

These analytical investigations are complemented by presenting a numerical algorithm based on $C^{1}$-elements and numerical studies. They intensively interact with geometric constructions in finding suitable minimising sequences for the Willmore functional.
\end{abstract}

Keywords. Dirichlet boundary conditions, Willmore surfaces of revolution.

AMS classification. 49Q10; 53C42, 35J65, 34L30.

\section{Contents}

1 Introduction $\quad \mathbf{2}$

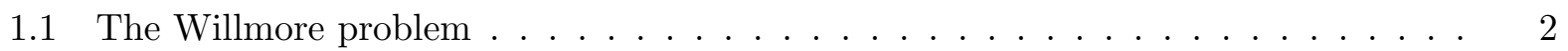

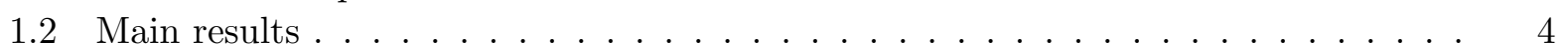

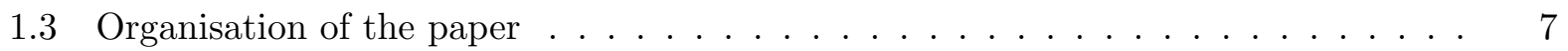

2 Geometric background $\quad 8$

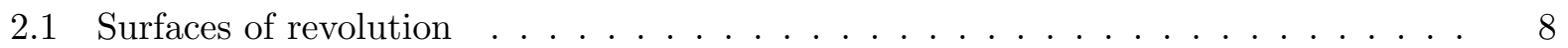

2.2 Surfaces of revolution as elastic curves in the hyperbolic half plane . . . . . . . . 8

2.3 Statement of the Willmore problem . . . . . . . . . . . . . . . . . 9

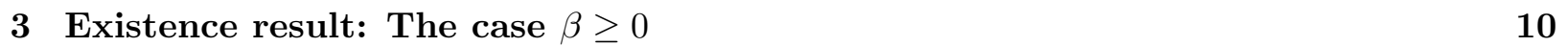

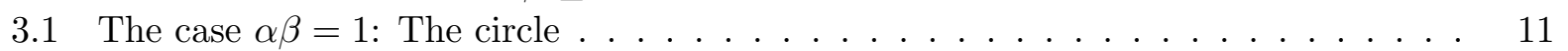

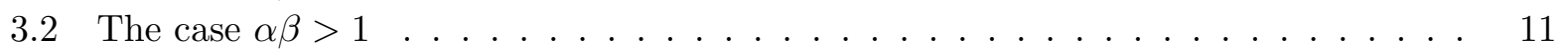

3.2.1 Monotonicity of the optimal energy . . . . . . . . . . . . . . . 11

3.2 .2 Properties of minimising sequences . . . . . . . . . . . . . . . . . . 13

*Financial support of "Deutsche Forschungsgemeinschaft" for the project "Randwertprobleme für Willmoreflächen - Analysis, Numerik und Numerische Analysis" (DE 611/5.1) is gratefully acknowledged 
3.2.3 Proof of the existence theorem . . . . . . . . . . . . . . . . . 14

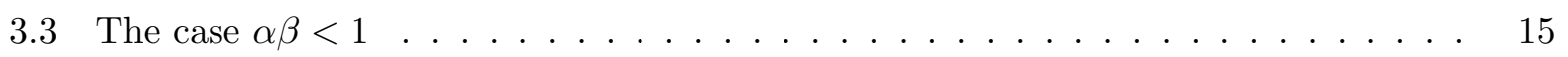

3.3 .1 Monotonicity of the optimal energy . . . . . . . . . . . . . . . . 15

3.3.2 Properties of minimising sequences . . . . . . . . . . . . . . . . 16

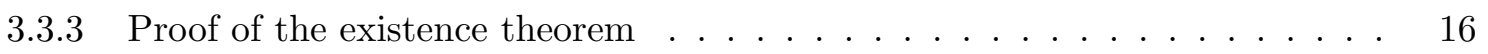

4 Existence result: The case $\beta<0 \quad 18$

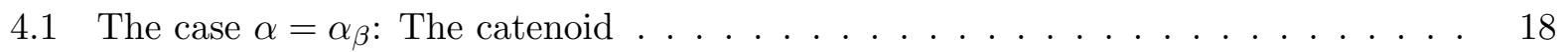

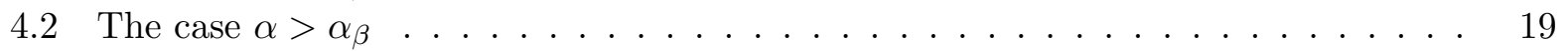

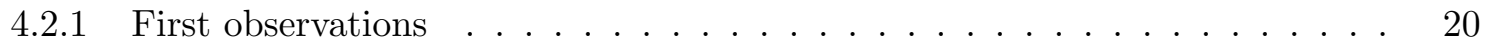

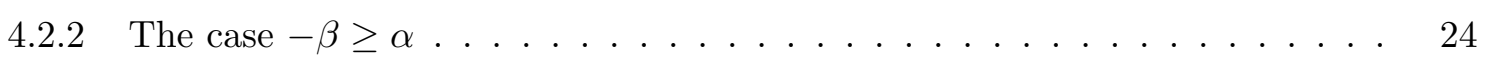

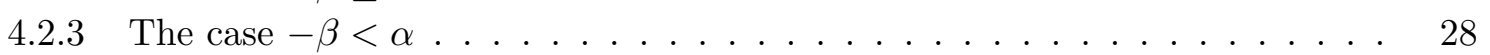

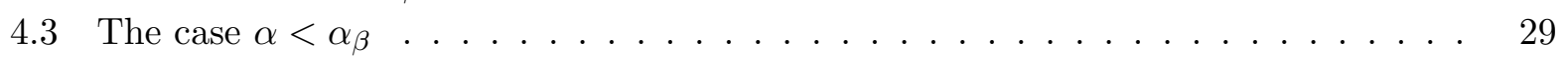

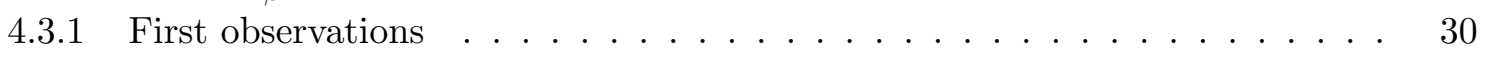

4.3.2 Monotonicity of the optimal energy . . . . . . . . . . . . . . 32

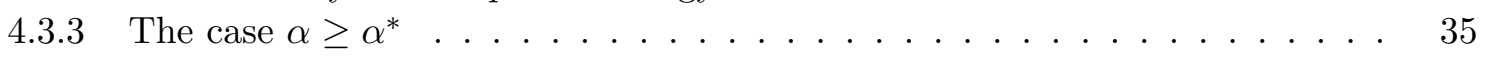

4.3 .4 The case $\alpha<\alpha^{*} \ldots \ldots \ldots \ldots \ldots \ldots \ldots \ldots \ldots$

5 Convergence to the sphere for $\alpha \searrow 0 \quad 43$

5.1 An upper bound for the energy as $\alpha \searrow 0 \ldots \ldots \ldots \ldots \ldots \ldots$

5.2 The limit of the energy . . . . . . . . . . . . . . . . . 44

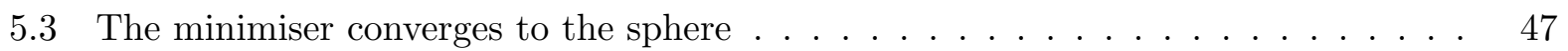

6 Qualitative properties of minimisers and estimates of the energy 49

6.1 The case $\alpha \beta>1 \ldots \ldots \ldots \ldots \ldots \ldots \ldots$

6.1 .1 Bounds on the energy . . . . . . . . . . . . . . . . . 49

6.1.2 On the sign of the hyperbolic curvature of minimisers . . . . . . . . . . 51

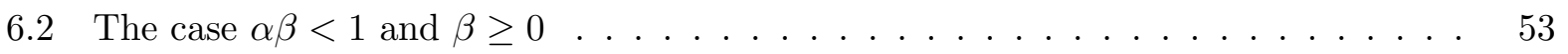

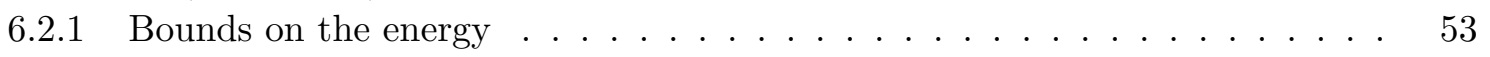

6.2 .2 On the sign of the hyperbolic curvature of minimisers $\ldots \ldots \ldots \ldots$

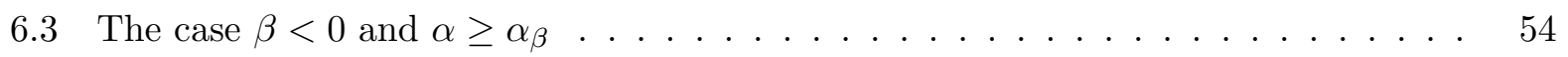

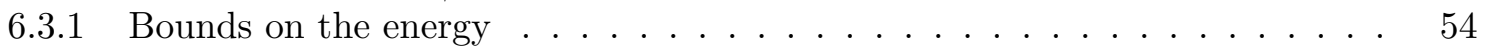

6.3.2 On the sign of the hyperbolic curvature of minimisers $\ldots \ldots \ldots \ldots$

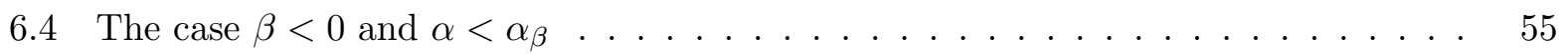

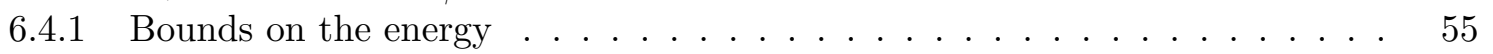

6.4.2 On the sign of the hyperbolic curvature of minimisers . . . . . . . . 56

$\begin{array}{lll}7 & \text { Numerical studies and algorithms } & 56\end{array}$

\section{Introduction}

\subsection{The Willmore problem}

Given a smooth and immersed surface $\Gamma \subset \mathbb{R}^{3}$, the Willmore functional is defined by

$$
\mathcal{W}(\Gamma):=\int_{\Gamma} H^{2} d A
$$

with $H$ the mean curvature of the immersion and $d A$ its area element. 
The functional $\mathcal{W}$ is of geometric interest, and it models the elastic energy of thin shells or biological membranes. It applies further in image processing and even in string theory (see e.g. [He, HP, Ni, KL, Z]). In these applications one is usually concerned with minima or, more generally, with critical points of the Willmore functional. Such a critical point $\Gamma \subset \mathbb{R}^{3}$ has to satisfy the Willmore equation

$$
\Delta_{\Gamma} H+2 H\left(H^{2}-K\right)=0 \quad \text { on } \Gamma,
$$

where $\Delta_{\Gamma}$ denotes the Laplace-Beltrami operator on $\Gamma$, and $K$ is the Gauss curvature of the surface. A solution of this non-linear fourth-order differential equation is called Willmore surface.

Although introduced already in the 19th century (see i.g. [P]), it was Willmore's work [Wi] which popularised again the investigation of the Willmore functional. Various existence and regularity results for closed Willmore surfaces of prescribed genus were extensively discussed in the literature. We want to mention in particular Bauer-Kuwert and Simon [BK, Sn] for existence of closed Willmore surfaces of prescribed genus, Kuwert-Schätzle and Leschke-Pedit-Pinkall $[\mathrm{KS} 1, \mathrm{KS} 2, \mathrm{LPP}]$ for closed Willmore surfaces of fixed conformal class and Rivière $[\mathrm{R}]$ for a far reaching regularity result. We refer to $[D D G]$ for a more extensive survey.

In the present paper we are interested in surfaces with boundaries. Therefore, we need to add to (1.2) appropriate boundary conditions. A discussion of possible choices can be found in Nitsche's survey article [Ni] along with corresponding existence results. Nitsche's results are based on perturbation arguments and require severe smallness conditions on the boundary data, which are by no means explicit. Furthermore, using methods from geometric measure theory, Schätzle proved in $[\mathrm{Sch}]$ existence and regularity of branched Willmore immersions in $\mathbb{S}^{n}$ with prescribed boundary conditions. By working in $\mathbb{S}^{n}$, some compactness problems could be overcome. On the other hand, when pulling back these immersions to $\mathbb{R}^{n}$ it cannot be excluded that they contain the point $\infty$. Due to the generality of his approach it seems to us that no topological information of the solution can be extracted from the existence proof. For numerical algorithms and numerical analysis for boundary value problems for the Willmore equation and the corresponding parabolic flow we mention Deckelnick, Droske, Rumpf and Dziuk (see [DD, DR, Dz] and references therein).

To prove existence of a priori bounded solutions to boundary value problems for the Willmore equation (1.2) with some specified further properties like e.g. the topological type or being a graph without imposing smallness conditions on the data seems to be a quite difficult task. Equation (1.2) is highly nonlinear and of fourth order and so, lacking any form of a general maximum or comparison principle. Most of the well established techniques from second order problems like e.g. the De Giorgi-Nash-Moser theory seem to break down completely in higher order problems. In order to start working on a theory of classical bounded smooth solutions for the Willmore boundary value problem we think that it is a good and appropriate strategy to investigate situations enjoying symmetry. Although then, one has an underlying ordinary differential equation, understanding solvability of the corresponding boundary value problems is by no means straightforward. In this spirit the one-dimensional Willmore problem or so called elastica were studied in [DG1, DG2]. Klaus Deckelnick and two of the authors investigated in [DDG] symmetric Willmore surfaces of revolution where the position and zero slope were prescribed on the boundary. By a number of refined geometric constructions it was possible to work with a priori bounded minimising sequences. Although the differential equation is one-dimensional, the geometry is to a large extent two-dimensional: Great difficulties arising from the interaction between the principal curvatures of the unknown surface are already present.

The previous work [DDG] was devoted to special Dirichlet boundary data. While the position at the boundary could be prescribed arbitrarily, one had to restrict to a zero boundary angle. Arbitrary boundary angles are subject of the present paper. 


\subsection{Main results}

In the present paper we will investigate a particular Dirichlet boundary value problem for (1.2). Namely, we consider surfaces of revolution $\Gamma \subset \mathbb{R}^{3}$ which are generated by rotating a smooth function $u:[-1,1] \rightarrow(0, \infty)$ about the $x=x_{1}$-axis. Then, $\Gamma$ can be parametrised as follows:

$$
(x, \varphi) \mapsto f(x, \varphi)=(x, u(x) \cos \varphi, u(x) \sin \varphi), \quad x \in[-1,1], \quad \varphi \in[0,2 \pi] .
$$

We consider the Willmore problem under symmetric Dirichlet boundary conditions where the height $u( \pm 1)=\alpha>0$ and an arbitrary angle $u^{\prime}(-1)=\beta=-u^{\prime}(1), \beta \in \mathbb{R}$, are prescribed at the boundary. The case $\beta=0$ has been studied in [DDG]. Our main result is the following.

Theorem 1.1 (Existence and regularity). For each $\alpha>0$ and each $\beta \in \mathbb{R}$, there exists a positive symmetric function $u \in C^{\infty}([-1,1],(0, \infty))$, i.e. $u(x)>0$ and $u(x)=u(-x)$, such that the corresponding surface of revolution $\Gamma \subset \mathbb{R}^{3}$ solves the Dirichlet problem for the Willmore equation

$$
\left\{\begin{array}{l}
\triangle_{\Gamma} H+2 H\left(H^{2}-K\right)=0 \quad \text { in }(-1,1), \\
u(-1)=u(+1)=\alpha, \quad u^{\prime}(-1)=-u^{\prime}(+1)=\beta .
\end{array}\right.
$$

The solution we find has the following additional properties:

1. If $\alpha \beta>1$, then $u^{\prime}<0$ in $(0,1]$ and $\left|u^{\prime}(x)\right| \leq \beta$ for all $x \in[-1,1]$.

2. If $\alpha \beta \leq 1$ and $\beta \geq 0$, then $u^{\prime}<0$ in $(0,1)$ and $\left|u^{\prime}(x)\right| \leq \frac{1}{\alpha}$ for all $x \in[-1,1]$.

3. If $\beta<0$ and $\alpha \operatorname{arsinh}(-\beta) \geq \sqrt{1+\beta^{2}}$, then $u^{\prime}>0$ in $(0,1]$.

4. If $\beta<0$ and $\alpha \operatorname{arsinh}(-\beta)<\sqrt{1+\beta^{2}}$, then $u$ has at most one critical point in $(0,1)$.

The proof is obtained by combining Theorems 3.11, 3.18, 4.17, 4.24, 4.39, 4.48 and Lemmas 3.1, 3.20 and 4.1 .

It may appear surprising that we find axially symmetric solutions of the Willmore boundary value problem for all values of $\alpha>0$ and $\beta \in \mathbb{R}$. For example, axially symmetric critical points of the area functional (i.e. minimal surfaces)

$$
\mathcal{A}(\Gamma)=2 \pi \int_{-1}^{1} u(x) \sqrt{1+u^{\prime}(x)^{2}} d x
$$

exist only for $u(1)=\alpha \geq \alpha^{*}$ where

$$
\alpha^{*}:=\frac{1}{b^{*}} \cosh \left(b^{*}\right)=1.5088795 \ldots
$$

and $b^{*}>0$ is the solution of the equation $\cosh \left(b^{*}\right)=b^{*} \sinh \left(b^{*}\right), b^{*}=1.1996786 \ldots$ Minimal surfaces of revolution, so called catenoids, are obtained for any $b \in(0, \infty)$ by rotating the curve $x \mapsto \frac{1}{b} \cosh (b x)$ around the $x$-axis. Not only for boundary data $\alpha \in\left(0, \alpha^{*}\right)$ these catenoids cease to exist, but according to [DHKW, Chapter 6.1, Theorem 3], there is no connected minimal surface solution at all - whether symmetric or not - for $\alpha<1$.

According to our result, for any set of symmetric Dirichlet boundary data, we always find at least one solution to the Willmore boundary value problem. For non-symmetric Dirichlet data - e.g. $u(1) \neq u(-1)$ - we expect a different picture. Analytical and numerical experiments suggest that one may be forced to impose conditions on the data $u(-1), u(1), u^{\prime}(-1), u^{\prime}(1)$ which deviate not too much from the symmetric setting. We feel that it might be even possible to prove nonexistence 
within the class of surfaces of revolutions generated by graphs for quite unsymmetric sets of data. For these data, however, existence may possibly still hold true in the class of parametric surfaces of revolution.

In order to prove our existence result Theorem 1.1, as in [DDG], we consider symmetric $C^{1,1}$ functions satisfying the boundary conditions and we study the minimisation problem in this class. In this setting, we prove that we may pass from arbitrary to suitable minimising sequences satisfying strong a priori bounds. We obtain these bounds by explicit geometric constructions which lower the Willmore energy. A key observation in doing so is the correspondence between the Willmore functional on surfaces of revolution and a curvature functional on curves in the hyperbolic half plane. The geometric constructions use geodesics of the hyperbolic half plane as well as catenoids, i.e. minimal surfaces of revolution. The obtained a priori bounds on the elements of the suitably modified minimising sequence ensure the required compactness and yield the desired existence result. In the setting of the hyperbolic half plane a classification of possible curvature functions in terms of elliptic functions of the arc length of the unknown curves is available, see [LS1, LS2]. However, we did not see a possibility to develop these results towards explicit formulae for boundary value problems (1.4). Moreover, we think that the geometric constructions performed in the present paper help to a good extent to understand the geometric shape of minimisers.

It remains as an interesting question whether these solutions minimise the Willmore energy also in the class of all immersed surfaces satisfying the same Dirichlet boundary conditions. For $\beta \neq 0$ and $\alpha \rightarrow \infty$ the energy bounds of Chapter 6 indicate that presumably this will not be the case. We expect that there might be parametric Willmore surfaces of revolution with much smaller Willmore energy.

Uniqueness is a further issue we have to leave open. We think that similarly as in the onedimensional analogous problem [DG1, Theorem 2] it should be possible to prove uniqueness and continuous dependence on data of energy minimising Willmore surfaces of revolution which are generated by graphs.

As can be seen from the statement of Theorem 1.1, the behaviour of those solutions of the Willmore equation constructed there depends not only on whether $\beta \geq 0$ or $\beta<0$. In both cases we have to make further distinctions. It seems that we have to treat all these cases separately. The switch between the different cases occurs when having explicit solutions. These solutions mark the values of the parameters where the qualitative behaviour of solutions changes. If $\alpha \beta=1$ then a solution is given by an arc of the circle with centre in the origin and going through the point $(1, \alpha)$. This is a geodesic in the hyperbolic half plane. The corresponding surface of revolution is part of a sphere which is the simplest possible closed Willmore surface. These geodesics of the hyperbolic half plane play an important role when studying the case $\beta \geq 0$. For $\beta<0$ and $\alpha \operatorname{arsinh}(-\beta)=\sqrt{1+\beta^{2}}$, the catenoid $u(x)=\cosh (b x) / b$ with $b=\operatorname{arsinh}(-\beta)$ is a minimal surface solution. Catenoids come into play in our constructions in addition to the hyperbolic geodesics when studying the case $\beta<0$. This interplay between two prototype Willmore surfaces gives rise to some technical difficulties. For $\beta<0$ and $|\beta|$ large, numerical calculations clearly display almost catenoidal and almost spherical (hyperbolically geodesic) parts of solutions.

Conformal invariance is a key feature of the Willmore functional and of Willmore surfaces. Rotation and translation are frequently employed, and scaling invariance is most important throughout the whole paper. On the other hand, inversions are not addressed here since in most cases they do not preserve the particular shape (1.3) of surfaces of revolution generated by graphs. Within this framework, only the relatively simple case $\alpha \beta>1$ could have been reduced to results in parts of the complementing cases, which are much more involved especially when $\beta<0$. In particular, boundary data with $\beta \leq \frac{1-\alpha^{2}}{2 \alpha}$ cannot be reduced to different cases because here, inversion does not yield graphs. But inversions are nevertheless quite interesting also here. Depending on $\alpha$, they directly yield parametric Willmore surfaces of revolution which are not generated by graphs 
for a range of tangent vectors pointing to the left in $x=-1$ and to the right in $x=1$. This is remarkable in so far as the general discussion of parametric surfaces of revolution is expected to be more difficult than that in the present paper.

Besides existence we also study further qualitative and asymptotic properties of solutions. A natural question is what happens to the solutions constructed in Theorem 1.1 when $\beta \in \mathbb{R}$ is fixed and $\alpha$ goes to 0 . We prove that they converge to the sphere centered at the origin with radius 1 .

Theorem 1.2. Fix $\beta \in \mathbb{R}$. For $\alpha>0$ let $u_{\alpha}$ be a solution to problem (1.4) as constructed in Theorem 1.1. Then, $u_{\alpha}$ converges for $\alpha \searrow 0$ to $x \mapsto \sqrt{1-x^{2}}$ in $C_{l o c}^{m}(-1,1)$ for any $m \in \mathbb{N}$.

For a proof see Theorem 5.8.

With our method of proving existence of solutions we get also information on the qualitative behaviour of the solutions. In particular, we can characterise the sign of the first derivative as stated in Theorem 1.1. Looking at the graph of a solution $u:[-1,1] \rightarrow(0, \infty)$ as a curve in the hyperbolic half plane, we study also the sign of its hyperbolic curvature. In Section 2.2 we recall some basic facts from hyperbolic geometry. However, the meaning of the sign of the hyperbolic curvature $\kappa_{h}[u](x)$ in $(x, u(x))$ is easily explained. One compares the graph of $u$ in $(x, u(x))$ with the tangential geodesic circle centered on the $x$-axis. Negative $\kappa_{h}[u](x)$ means that the graph is locally inside this circle while $\kappa_{h}[u](x)>0$ means that the graph of $u$ is locally outside this circle. Concerning the sign of the hyperbolic curvature of our solutions we have the following result. We skip the case $\alpha \beta=1$, where the solution is a geodesic circle.

Theorem 1.3. For $\alpha>0$ and $\beta \in \mathbb{R}$ let $u \in C^{\infty}([-1,1],(0, \infty))$ be a solution to problem (1.4) as constructed in Theorem 1.1. Let $\kappa_{h}[u]$ denote the hyperbolic curvature of the curve $\{(x, u(x))$ : $x \in[-1,1]\}$. Then, $\kappa_{h}[u]$ has the following sign properties:

1. If $\alpha \beta>1$, then $\kappa_{h}[u](0)<0$ and $\kappa_{h}[u]$ has at most one change of sign in $(0,1)$.

2. If $\alpha \beta<1$ and $\beta \geq 0$, then $\kappa_{h}[u]>0$ in $(-1,1)$.

3. If $\beta<0$ and $\alpha \operatorname{arsinh}(-\beta)>\sqrt{1+\beta^{2}}$, then $\kappa_{h}[u](0)>0$ and $\kappa_{h}[u]$ has at most one change of sign in $(0,1)$.

4. If $\beta<0$ and $\alpha \operatorname{arsinh}(-\beta) \leq \sqrt{1+\beta^{2}}$, then $\kappa_{h}[u]>0$ in $(-1,1)$.

The proof is obtained by combining Theorems $6.4,6.7,6.9$ and 6.11 .

Numerical calculations give evidence to our feeling that in the case $\alpha \beta>1$ the hyperbolic curvature may indeed have a change of sign.

It is not only in this respect that the analytical investigations of the present paper benefit a lot from numerical simulations. Numerically calculated solutions help in finding qualitative properties of suitable minimising sequences while, at the same time, analytical insights help to identify suitable initial data such that the numerical gradient flow method indeed converges. In Chapter 7 , we explain a $C^{1}$-finite element method, which we think is natural in order to deal with Dirichlet boundary conditions. It seems that so far, no $C^{1}$-finite element algorithms are available for Willmore surfaces. Like in the analytic part we consider the present paper as a first step also in numerical investigations of Dirichlet problems. We are confident that, basing upon these experiences, we may develop $C^{1}$-finite element algorithms also for graphs e.g. over general two-dimensional domains. This will be subject of future research.

We remark that in particular the Navier boundary value problem is numerically well investigated, where the position of the surface and its mean curvature are prescribed at the boundary. See e.g. $[\mathrm{DD}, \mathrm{Dz}]$ and references therein. In this case the Willmore boundary value problem may 
be written as a second order system for the position and the mean curvature and continuous finite elements may be used.

Droske and Rumpf [DR] proposed a level set formulation for the Dirichlet problem and for closed Willmore surfaces and developed a corresponding piecewise linear continuous finite element algorithm.

\subsection{Organisation of the paper}

In Chapter 2 we recall some basic geometric notions which are relevant for our analysis, and formulate the minimisation problem for the Willmore functional as we shall study it. We explain that the Willmore functional for surfaces of revolution $\Gamma$ as in (1.3) corresponds to a functional defined on curves in the hyperbolic half plane. We call this second functional the "hyperbolic Willmore functional". This observation was already made by Pinkall and Bryant-Griffiths and used in [Br, BG, LS2, DDG].

In Chapter 3 we prove Theorem 1.1 in the case $\beta \geq 0$ taking advantage of the reformulation of the minimisation problem in the hyperbolic half plane. For $\alpha \beta=1$ we have a part of a sphere as an explicit solution. We distinguish then the cases $\alpha \beta>1$ and $\alpha \beta<1$. In both cases we first prove monotonicity of the energy. The energy is increasing in $\alpha$ for $\alpha \beta>1$, while it is decreasing in $\alpha$ for $\alpha \beta<1$. By geometric constructions we prove that we can restrict ourselves to minimising sequences satisfying strong a priori bounds, which are as in Theorem 1.1, Properties 1 and 2 respectively. The key ingredient is to insert suitable parts of hyperbolic geodesic circles. The case $\alpha \beta<1$ may be viewed as a direct generalisation of the result for $\beta=0$ from [DDG]. As for estimates and existence we proceed exactly like there and are quite brief here for this reason. However, we improve it by showing that our solution even satisfies $u^{\prime}<0$ in $(0,1)$. Obtaining a priori estimates in the case $\alpha \beta>1$ is more involved since the geodesic circle through the boundary points does no longer serve as a comparison function.

In Chapter 4 we prove Theorem 1.1 in the case $\beta<0$. For $\alpha=\alpha_{\beta}:=\sqrt{1+\beta^{2}} / \operatorname{arsinh}(-\beta)$ a solution is the catenoid $x \mapsto \cosh (b x) / b$ with $b=\operatorname{arsinh}(-\beta)$. Then, we distinguish the cases $\alpha>\alpha_{\beta}$ and $\alpha<\alpha_{\beta}$. Here, the requisite geometric constructions in order to achieve strong enough a priori information on suitably modified minimising sequences do not only involve the hyperbolic geodesics but also the catenoids as minimal surfaces of revolution. These constructions are different not only according to the cases $\alpha>\alpha_{\beta}$ and $\alpha<\alpha_{\beta}$, but depend also on whether $-\beta \geq \alpha$ or $-\beta<\alpha$ and whether $\alpha \geq \alpha^{*}$ or $\alpha<\alpha^{*}$. The parameter $\alpha^{*}=\min \{\cosh (b) / b: b \in(0, \infty)\}$ refers to the smallest boundary height where for some boundary angle one may have a catenoid as solution. If $|\beta|$ becomes large and $\alpha$ small it turns out to be somehow delicate to prevent minimising sequences from getting too close to 0 and to obtain bounds from below. Surprisingly, the case where $\alpha>\alpha_{\beta}$ and $-\beta<\alpha$ is special, because here we can prevent a possible loss of compactness only by further restricting the class of admissible functions.

In Chapter 5 we study the behaviour of minimisers for $\alpha \searrow 0$. We prove that our minimisers converge locally uniformly in $(-1,1)$ to the sphere. In Chapter 6 we prove bounds on the Willmore energy and we study the sign of the hyperbolic curvature of the constructed solutions.

Chapter 7 gives a description of a $C^{1}$-finite element algorithm for the underlying Willmore gradient flow. Moreover, numerical studies are performed, and we provide a series of pictures illustrating typical shapes of solutions within different parameter regimes. 


\section{Geometric background}

\subsection{Surfaces of revolution}

We consider any function $u \in C^{4}([-1,1],(0, \infty))$. Rotating the curve $(x, u(x)) \subset \mathbb{R}^{2}$ about the $x$-axis generates a surface of revolution $\Gamma \subset \mathbb{R}^{3}$ which can be parametrised by

$$
\Gamma: f(x, \varphi)=(x, u(x) \cos \varphi, u(x) \sin \varphi) \in \mathbb{R}^{3}, \quad x \in[-1,1], \varphi \in[0,2 \pi) .
$$

The term "surface" always refers to the mapping $f$ as well as to the set $\Gamma$. The condition $u>0$ implies that $f$ is embedded in $\mathbb{R}^{3}$ and in particular immersed.

Let $\kappa_{1}$ and $\kappa_{2}$ denote the principal curvatures of the surface $\Gamma \subset \mathbb{R}^{3}$, that is $\kappa_{1}=-u^{\prime \prime}(x)(1+$ $\left.u^{\prime}(x)^{2}\right)^{-\frac{3}{2}}$ and $\kappa_{2}=\left(u(x) \sqrt{1+u^{\prime}(x)^{2}}\right)^{-1}$. Its mean curvature $H$ and Gaussian curvature $K$ are

$$
\begin{aligned}
H & :=\frac{\kappa_{1}+\kappa_{2}}{2}=-\frac{u^{\prime \prime}(x)}{2\left(1+u^{\prime}(x)^{2}\right)^{3 / 2}}+\frac{1}{2 u(x) \sqrt{1+u^{\prime}(x)^{2}}}=\frac{1}{2 u(x) u^{\prime}(x)}\left(\frac{u(x)}{\sqrt{1+u^{\prime}(x)^{2}}}\right)^{\prime}, \\
K & :=\kappa_{1} \kappa_{2}=-\frac{u^{\prime \prime}(x)}{u\left(1+u^{\prime}(x)^{2}\right)^{2}} .
\end{aligned}
$$

The Willmore energy of $\Gamma$ defined in (1.1) is the integral over the surface of the mean curvature squared. In particular, written in terms of the function $u$ it has the form

$$
\mathcal{W}(\Gamma)=\frac{\pi}{2} \int_{-1}^{1}\left(\frac{u^{\prime \prime}(x)}{\left(1+u^{\prime}(x)^{2}\right)^{3 / 2}}-\frac{1}{u(x) \sqrt{1+u^{\prime}(x)^{2}}}\right)^{2} u(x) \sqrt{1+u^{\prime}(x)^{2}} d x
$$

\subsection{Surfaces of revolution as elastic curves in the hyperbolic half plane}

Following $[\mathrm{Br}, \mathrm{BG}]$, the construction of axially symmetric critical points $\Gamma$ of the Willmore functional can be reduced to finding elastic curves in the upper half-plane $\mathbb{R}_{+}^{2}:=\left\{(x, y) \in \mathbb{R}^{2}: y>0\right\}$ equipped with the hyperbolic metric $d s_{h}^{2}:=\frac{1}{y^{2}}\left(d x^{2}+d y^{2}\right)$. Geodesics are circular arcs centered on the $x$-axis and lines parallel to the $y$-axis; the first will play a crucial role in this work.

Let $s \mapsto \gamma(s)=\left(\gamma_{1}(s), \gamma_{2}(s)\right)$, where we do not raise the indices, be a curve in $\mathbb{R}_{+}^{2}$ parametrised with respect to its arc length, i.e.

$$
1 \equiv \frac{\gamma_{1}^{\prime}(s)^{2}+\gamma_{2}^{\prime}(s)^{2}}{\gamma_{2}(s)^{2}}
$$

Then, its curvature is given by

$$
\kappa_{h}(s)=-\frac{\gamma_{2}(s)^{2}}{\gamma_{2}^{\prime}(s)} \frac{d}{d s}\left(\frac{\gamma_{1}^{\prime}(s)}{\gamma_{2}(s)^{2}}\right)=\frac{\gamma_{2}(s)^{2}}{\gamma_{1}^{\prime}(s)}\left(\frac{1}{\gamma_{2}(s)}+\frac{d}{d s}\left(\frac{\gamma_{2}^{\prime}(s)}{\gamma_{2}(s)^{2}}\right)\right) .
$$

For graphs $[-1,1] \ni x \mapsto(x, u(x)) \in \mathbb{R}_{+}^{2}$, formula (2.2) yields

$$
\kappa_{h}[u](x)=-\frac{u(x)^{2}}{u^{\prime}(x)} \frac{d}{d x}\left(\frac{1}{u(x) \sqrt{1+u^{\prime}(x)^{2}}}\right)=\frac{u(x) u^{\prime \prime}(x)}{\left(1+u^{\prime}(x)^{2}\right)^{3 / 2}}+\frac{1}{\sqrt{1+u^{\prime}(x)^{2}}} .
$$

Using identity (2.3), we compute for the squared hyperbolic curvature times the hyperbolic line 
element:

$$
\begin{aligned}
\kappa_{h}[u]^{2} \frac{\sqrt{1+u^{\prime 2}}}{u} & =\left\{\frac{u^{\prime \prime}}{\left(1+u^{\prime 2}\right)^{3 / 2}}+\frac{1}{u \sqrt{1+u^{\prime 2}}}\right\}^{2} u \sqrt{1+u^{\prime 2}} \\
& =\left\{\frac{u^{\prime \prime}}{\left(1+u^{\prime 2}\right)^{3 / 2}}-\frac{1}{u \sqrt{1+u^{\prime 2}}}\right\}^{2} u \sqrt{1+u^{\prime 2}}+4 \frac{u^{\prime \prime}}{\left(1+u^{\prime 2}\right)^{\frac{3}{2}}} \\
& =4 H^{2} u \sqrt{1+u^{\prime 2}}+4 \frac{u^{\prime \prime}}{\left(1+u^{\prime 2}\right)^{\frac{3}{2}}} .
\end{aligned}
$$

We define the hyperbolic Willmore energy as the elastic energy of the graph of $u$ in the hyperbolic half plane and compare it with the original Willmore functional $\mathcal{W}(\Gamma)$ defined in $(2.1)$.

$$
\mathcal{W}_{h}(u):=\int_{\gamma} \kappa_{h}[u]^{2} d s_{h}[u]:=\int_{-1}^{1} \kappa_{h}[u]^{2} \frac{\sqrt{1+u^{\prime 2}}}{u} d x=\frac{2}{\pi} \mathcal{W}(\Gamma)+4 \int_{-1}^{1} \frac{u^{\prime \prime}}{\left(1+u^{\prime 2}\right)^{3 / 2}} d x
$$

where $\Gamma$ is the surface of revolution obtained by rotating the graph of $u$.

Lemma 2.1 (Duality of $\mathcal{W}_{h}(u)$ and $\mathcal{W}(\Gamma)$ ). The hyperbolic energy $\mathcal{W}_{h}(u)$ of a curve $u \in \mathbb{R}_{+}^{2}$ and the Willmore energy of the corresponding surface of revolution $\Gamma \subset \mathbb{R}^{3}$ satisfy

$$
\mathcal{W}_{h}(u)=\frac{2}{\pi} \mathcal{W}(\Gamma)+4\left[\frac{u^{\prime}(x)}{\sqrt{1+u^{\prime}(x)^{2}}}\right]_{-1}^{1} .
$$

This observation goes back to Pinkall and Bryant-Griffiths [Br, BG], see also [LS1, LS2]. The present derivation is adapted from [DDG, Section 2.2].

In proving Theorem 1.1, we benefit a lot from this duality between the Willmore functional and the hyperbolic Willmore energy. We do not only take technical advantage from this result, but we think that switching between both functionals helps to a good extent in understanding underlying geometric features.

Concerning the Euler-Lagrange equation for critical points of the hyperbolic Willmore functional $\mathcal{W}_{h}$ one has:

Lemma 2.2. Assume that $u \in C^{4}([-1,1],(0, \infty))$ is such that for all $\varphi \in C_{0}^{\infty}([-1,1],(0, \infty))$ one has that $0=\left.\frac{d}{d t} \mathcal{W}_{h}(u+t \varphi)\right|_{t=0}$. Then, $u$ satisfies the following Euler-Lagrange equation

$$
\frac{u(x)}{\sqrt{1+u^{\prime}(x)^{2}}} \frac{d}{d x}\left(\frac{u(x)}{\sqrt{1+u^{\prime}(x)^{2}}} \kappa_{h}^{\prime}(x)\right)-\kappa_{h}(x)+\frac{1}{2} \kappa_{h}(x)^{3}=0, \quad x \in(-1,1),
$$

with $\kappa_{h}=\kappa_{h}[u]$ as defined in (2.3).

When parametrised by the hyperbolic arc length $s$, equation (2.5) takes the simple form $\frac{d^{2}}{d s^{2}} \kappa_{h}(s)-\kappa_{h}(s)+\frac{1}{2} \kappa_{h}(s)^{3}=0$. This equation was discussed in detail in [LS1] and curvatures of solutions were classified in terms of elliptic functions of the hyperbolic arc length $s$. However, we do not see any possibility to solve directly and explicitly our Willmore boundary value problem (1.4) basing upon this classification.

\subsection{Statement of the Willmore problem}

The Willmore boundary value problem (1.4) will be solved by minimising the hyperbolic Willmore functional within the following class of functions: 
Definition 2.3. For $\alpha>0$ and $\beta \in \mathbb{R}$ we introduce the function space

$$
N_{\alpha, \beta}:=\left\{u \in C^{1,1}([-1,1],(0, \infty)): u \text { positive, symmetric, } u(1)=\alpha \text { and } u^{\prime}(-1)=\beta\right\}
$$

as well as

$$
M_{\alpha, \beta}:=\inf \left\{\mathcal{W}_{h}(u): u \in N_{\alpha, \beta}\right\}
$$

Lemma 2.1 gives that

$$
\mathcal{W}(\Gamma)=\frac{\pi}{2} \mathcal{W}_{h}(u)+4 \pi \frac{\beta}{\sqrt{1+\beta^{2}}}
$$

for the surface $\Gamma$ of revolution generated by $u \in N_{\alpha, \beta}$. Since we are working with Dirichlet boundary conditions we may switch between the two functionals depending on which one is more convenient.

In the following sections we will prove existence of solutions $u_{\alpha, \beta} \in N_{\alpha, \beta} \cap C^{\infty}([-1,1], \mathbb{R})$ such that $\mathcal{W}_{h}\left(u_{\alpha, \beta}\right)=M_{\alpha, \beta}$. Only in the case of parameters treated in Subsection 4.2.3, $N_{\alpha, \beta}$ has for technical reasons to be replaced by a smaller set of admissible functions. The axially symmetric surface $\Gamma_{\alpha, \beta}$ which is generated by $u_{\alpha, \beta}$ is solution of the Willmore boundary value problem (1.4). See [DG3, Lemma 1] for an elementary calculation of the Euler-Lagrange equation in this particular setting. For a general survey on the Willmore functional, corresponding Euler-Lagrange equations and natural boundary conditions we refer to the survey article by Nitsche [Ni], cf. also [Th, p. 56]. The Euler-Lagrange equation for the Willmore functional in nonparametric form was already discussed by Poisson [P, p. 224].

Remark 2.4. The Willmore energy is invariant under rescaling. I.e. if $u$ is a positive function in $C^{1,1}([-r, r],(0, \infty))$ for some $r>0$, then the function $v \in C^{1,1}([-1,1],(0, \infty))$ defined by $v(x)=u(r x) / r$ has the same hyperbolic Willmore energy as $u$, that is,

$$
\mathcal{W}_{h}(v)=\int_{-1}^{1} \kappa_{h}^{2}[v] d s_{h}[v]=\int_{-r}^{r} \kappa_{h}^{2}[u] d s_{h}[u] .
$$

Here, $\kappa_{h}[u]$ is the hyperbolic curvature of $u$ as defined in $(2.3)$ and $\mathcal{W}_{h}(v)$ is the hyperbolic Willmore energy of $v$ as defined in (2.4).

\section{Existence result: The case $\beta \geq 0$}

In this section we consider $\beta \geq 0$ and keep it fixed, while $\alpha$ varies in the positive real numbers.

For the value of $\alpha$ such that $\alpha \beta=1$ we have an explicit solution of (1.4). This is the arc of the circle with centre at the origin and going through the point $(1, \alpha)$. This solution is in particular a geodesic curve in the hyperbolic half plane. It marks the point where there is a change in the behaviour of the energy. For $\alpha \beta>1$ the energy $M_{\alpha, \beta}$, defined in (2.7), is monotonically increasing in $\alpha$, while for $\alpha \beta<1$ it is monotonically decreasing in $\alpha$.

Minimising sequences are suitably modified by means of parts of geodesic circles in order to achieve strong enough a priori estimates ensuring compactness. In this respect the case $\alpha \beta>1$ is more involved than the case $\alpha \beta<1$, because here there is no canonical comparison function from above. However, one can pass to minimising sequences where the derivative is maximal in $x=-1$. The case $\alpha \beta<1$ is quite similar to and contains the main result Theorem 1.1 from the previous work [DDG] as a special case. However, this simplicity is due to referring to its main geometric construction. Here, a geodesic circle provides an obvious upper bound. Moreover, we prove an extra property of the solution $u$ constructed there, namely that $u^{\prime}(x)<0$ on $(0,1)$. 


\subsection{The case $\alpha \beta=1$ : The circle}

Here, we have an explicit solution.

Lemma 3.1. For each $\alpha>0$ and $\beta$ such that $\alpha \beta=1$, the part of the sphere $\Gamma \subset \mathbb{R}^{3}$ generated as a surface of revolution by the function $u(x)=\sqrt{1+\alpha^{2}-x^{2}}, x \in[-1,1]$ solves the Dirichlet problem (1.4).

Moreover, the corresponding surface of revolution is the unique minimiser of the Willmore functional (1.1) among all axially symmetric surfaces generated by graphs of symmetric functions in $C^{1,1}([-1,1],(0, \infty))$ such that $v( \pm 1)=\alpha$ and $v^{\prime}(1)=-\beta$.

Proof. Since $\kappa_{h}[u] \equiv 0$ in $[-1,1]$, the claim follows from Lemma 2.1 and the definition of the hyperbolic Willmore functional in (2.4).

\subsection{The case $\alpha \beta>1$}

\subsubsection{Monotonicity of the optimal energy}

In this paragraph we prove that the Willmore energy is increasing in $\alpha$. The proof is divided into the next four lemmas. First, we prove that it is enough to consider functions in $N_{\alpha, \beta}$ which are decreasing in $[0,1]$. The proof will refer to a main result of the previous work [DDG, Theorem 3.8], which involves a number of refined geometric constructions. We emphasise that obvious constructions like reflections do not yield the following result.

Lemma 3.2. For each $u \in N_{\alpha, \beta}$ with only finitely many critical points, we find a function $v \in N_{\alpha, \beta}$ having at most as many critical points as $u$, with lower Willmore energy than $u$ and satisfying $v^{\prime}(x) \leq 0$ for all $x \in[0,1]$.

Proof. Assume that $u$ does not have the claimed property. Then, there exists $x_{0} \in(0,1)$ such that $\left[-x_{0}, x_{0}\right]$ is the largest possible symmetric interval with the property $u^{\prime}\left(x_{0}\right)=0$ and $u^{\prime}(x)<0$ in $\left(x_{0}, 1\right]$. Using a rescaled version of [DDG, Theorem 3.8] we substitute $\left.u\right|_{\left[-x_{0}, x_{0}\right]}$ by a symmetric positive $C^{1,1}$-function defined on the same interval, having the same boundary values as $u$ in $x_{0}$, having lower Willmore energy than $\left.u\right|_{\left[-x_{0}, x_{0}\right]}$, having at most as many critical points as $\left.u\right|_{\left[-x_{0}, x_{0}\right]}$ and decreasing in $\left[0, x_{0}\right]$. The so obtained function $v$ is element of $N_{\alpha, \beta}$, it has at most as many critical points as $u, \mathcal{W}_{h}(v) \leq \mathcal{W}_{h}(u)$ and $v^{\prime}(x) \leq 0$ in $[0,1]$.

In the proof we need only that $\beta>0$. Notice further that one could substitute $\left.u\right|_{\left[-x_{0}, x_{0}\right]}$ with an appropriately rescaled solution of the Willmore problem with $\beta=0$ and height $u\left(x_{0}\right) / x_{0}$ as constructed in [DDG, Theorem 1.1]. This statement, however, does not give control of the number of critical points. With arguments introduced below we shall see - a posteriori - that we could indeed achieve $u^{\prime}<0$ on $\left(0, x_{0}\right)$.

In the next lemma, we construct for any $u \in N_{\alpha, \beta}$ which is decreasing in $[0,1]$ a function with the same boundary values having lower Willmore energy than $u$ and being defined in a larger interval.

Lemma 3.3. Assume that $u \in N_{\alpha, \beta}$ has only finitely many critical points and satisfies $u^{\prime}(x) \leq 0$ for all $x \in[0,1]$. Then, for each $\varrho \in[1, \alpha \beta)$, there exists a positive and symmetric function $u_{\varrho} \in C^{1,1}([-\varrho, \varrho],(0, \infty))$ such that $u_{\varrho}(\varrho)=\alpha, u_{\varrho}^{\prime}(\varrho)=-\beta, u_{\varrho}^{\prime}(x) \leq 0$ for all $x \in[0, \varrho]$, $u_{\varrho}$ has at most as many critical points as $u$, and, furthermore, one has

$$
\int_{-\varrho}^{\varrho} \kappa_{h}\left[u_{\varrho}\right]^{2} d s_{h}\left[u_{\varrho}\right] \leq \mathcal{W}_{h}(u) \text {. }
$$


Proof. The construction is similar to the one of [DDG, Lemma 3.3]. The situation there differs from the present one in the non-vanishing boundary conditions for $u^{\prime}$. There we decrease the energy by shortening the interval, while here it is elongated.

Let $r \in(0,1)$ be a parameter. The (euclidian) normal to the graph of $u$ in $(r, u(r))$ has direction $\left(-u^{\prime}(r), 1\right)$. The straight line generated by this normal intersects the $x$-axis left of $r$, since $u$ is decreasing. We take this intersection point $(c(r), 0)$ as centre for a geodesic circular arc, where the radius is chosen such that this arc is tangential to the graph of $u$ in $(r, u(r))$. In particular, the radius is given by the distance between $(c(r), 0)$ and $(r, u(r))$. We build a new symmetric function with smaller hyperbolic curvature integral as follows: On $[c(r), r]$ we take this geodesic arc, which has horizontal tangent in $c(r)$, while on $[r, 1]$ we take $u$. By construction, this function is $C^{1,1}([c(r), 1],(0, \infty))$ and decreasing. We shift it such that $c(r)$ is moved to 0 , and extend this to an even function, which is again $C^{1,1}$, now on a suitable interval $[-\ell(r), \ell(r)]$, with $\ell(r)=1-c(r)$ and $c(r)=r+u(r) u^{\prime}(r)$. This new function has the same boundary values as $u$, at most as many critical points as $u$, and, by construction, a smaller curvature integral. Our construction yields the claim since $r \mapsto \ell(r)$ is continuous and such that $\lim _{r \backslash 0} \ell(r)=1$ and $\lim _{r}{ }_{11} \ell(r)=\alpha \beta$.

Remark 3.4. Notice that, by concavity of the geodesic circles, $u_{\varrho}^{\prime}(x) \geq-\gamma$ in $[0, \varrho]$ if $u^{\prime}(x) \geq-\gamma$ in $[0,1]$.

a)

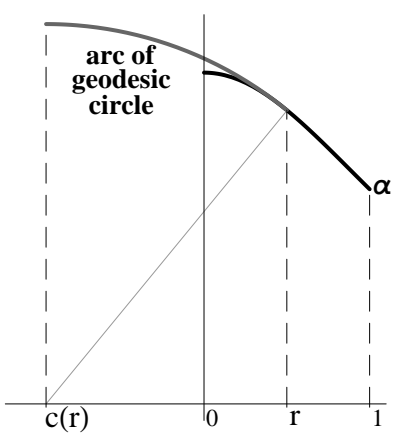

b)

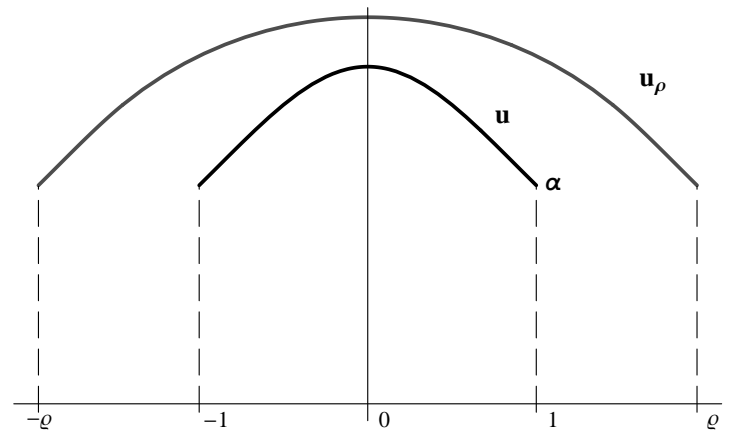

Figure 1: Proof of Lemma 3.3.

By Lemma 3.2, we can remove the assumption that $u^{\prime}(x) \leq 0$ in $[0,1]$ from Lemma 3.3.

Lemma 3.5. Assume that $u \in N_{\alpha, \beta}$ has only finitely many critical points. Then, for each $\varrho \in$ $[1, \alpha \beta)$, there exist a positive and symmetric function $u_{\varrho} \in C^{1,1}([-\varrho, \varrho],(0, \infty))$ such that $u_{\varrho}(\varrho)=\alpha$, $u_{\varrho}^{\prime}(\varrho)=-\beta, u_{\varrho}^{\prime}(x) \leq 0$ for all $x \in[0, \varrho], u_{\varrho}$ has at most as many critical points as $u$, and, furthermore, it holds that

$$
\int_{-\varrho}^{\varrho} \kappa_{h}\left[u_{\varrho}\right]^{2} d s_{h}\left[u_{\varrho}\right] \leq \mathcal{W}_{h}(u) .
$$

Proof. By Lemma 3.2 there exists $v$ in $N_{\alpha, \beta}$ having at most as many critical points as $u$, with lower Willmore energy than $u$ and satisfying $v^{\prime}(x) \leq 0$ in $[0,1]$. The claim follows from Lemma 3.3 applied to $v$.

By rescaling we obtain:

Lemma 3.6. For each $u \in N_{\alpha, \beta}$ having only finitely many critical points and for each $\gamma \in\left[\beta^{-1}, \alpha\right]$ there exists a symmetric function $v \in C^{1,1}([-1,1],(0, \infty))$ having at most as many critical points as $u$ and satisfying: $v( \pm 1)=\gamma, v^{\prime}(1)=-\beta, v^{\prime}(x) \leq 0$ in $[0,1]$ and $\mathcal{W}_{h}(v) \leq \mathcal{W}_{h}(u)$. 
Proof. If $\gamma \in\left(\beta^{-1}, \alpha\right]$ the claim follows from Lemma 3.5 by rescaling. If $\gamma=\beta^{-1}$ we choose $v(x):=\sqrt{1+\gamma^{2}-x^{2}}$.

The previous lemma gives that the optimal Willmore energy $M_{\alpha, \beta}$, defined in (2.7), is increasing in $\alpha$.

Proposition 3.7. We have $M_{\widetilde{\alpha}, \beta} \geq M_{\alpha, \beta}$ for all $\alpha$, $\widetilde{\alpha}$ such that $\widetilde{\alpha} \geq \alpha \geq \frac{1}{\beta}$.

Proof. Since polynomials are dense in $H^{2}(-1,1)$, a minimising sequence for $M_{\tilde{\alpha}, \beta}$ may be chosen in $N_{\tilde{\alpha}, \beta}$, which consists of symmetric and positive polynomials. Lemma 3.6 proves the statement.

In Proposition 6.2 we prove that even $\lim _{\alpha \rightarrow \infty} M_{\alpha, \beta}=+\infty$.

\subsubsection{Properties of minimising sequences}

In the next two lemmas we introduce geometric constructions and show that on minimising sequences, by possibly inserting parts of geodesic circles and rescaling, we may assume that $0 \geq u^{\prime}(x) \geq-\beta$ and $x+u(x) u^{\prime}(x) \leq 0$ for $x \in[0,1]$.

We first employ the elongation procedure of Lemma 3.3 and rescaling to achieve the derivative bounds.

Lemma 3.8. For each $u \in N_{\alpha, \beta}$ with only finitely many critical points there exists $v \in N_{\alpha, \beta}$ having at most as many critical points as $u$, with lower Willmore energy than $u$ and such that

$$
-\beta \leq v^{\prime}(x) \leq 0 \text { for all } x \in[0,1] .
$$

Proof. By Lemma 3.2 there exists $w \in N_{\alpha, \beta}$ having at most as many critical points as $u$ with lower Willmore energy than $u$ and such that $w^{\prime}(x) \leq 0$ in $[0,1]$. If moreover, $w^{\prime}(x) \geq-\beta$ the claim follows with $v=w$. Otherwise there exists a first $x_{1} \in(0,1)$ with $w^{\prime}\left(x_{1}\right)=-\beta$ such that in particular $w^{\prime}(x) \geq-\beta$ on $\left[0, x_{1}\right]$. By using a scaled version of Lemma 3.3 we dilate the function $\left.w\right|_{\left[-x_{1}, x_{1}\right]}$ by inserting an arc of a geodesic circle. For each $\varrho \in\left(x_{1}, w\left(x_{1}\right) \beta\right)$ there exists $w_{\varrho} \in C^{1,1}([-\varrho, \varrho],(0, \infty))$ with lower Willmore energy than $\left.w\right|_{\left[-x_{1}, x_{1}\right]}$, with at most as many critical points as $\left.w\right|_{\left[-x_{1}, x_{1}\right]}$ and such that $w_{\varrho}( \pm \varrho)=w\left(x_{1}\right)$ and $w_{\varrho}^{\prime}(\varrho)=-\beta$. Notice that by concavity of the geodesic circles $w_{\varrho}^{\prime}(x) \geq-\beta$ in $[0, \varrho]$. We choose $\varrho=w\left(x_{1}\right) / \alpha$ and $v$ to be equal to $w_{\varrho}$ being rescaled to the interval $[-1,1]$. The choice of $\varrho$ is such that we dilate the graph of $\left.w\right|_{\left[0, x_{1}\right]}$ until we reach the line $y \mapsto \alpha y$. This construction is illustrated in Figure 2 .

a)

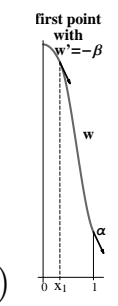

b)

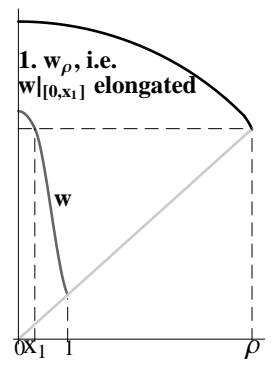

c)

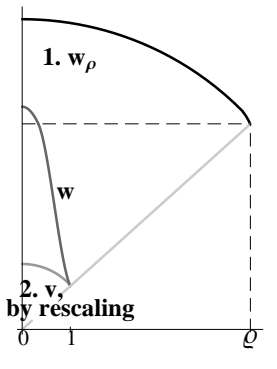

Figure 2: Proof of Lemma 3.8.

We now add the property $x+u(x) u^{\prime}(x) \leq 0$ for $x \in[0,1]$ to those of the previous lemma by possibly inserting a suitable part of a geodesic circle. 
Lemma 3.9. For each $u \in N_{\alpha, \beta}$ having only finitely many critical points, there exists $v \in N_{\alpha, \beta}$ having at most as many critical points as u, with lower Willmore energy than $u$ and satisfying

$$
0 \geq v^{\prime}(x) \geq-\beta \text { and } 0 \geq x+v(x) v^{\prime}(x) \text { for all } x \in[0,1] .
$$

Proof. By Lemma 3.8 there exists $w \in N_{\alpha, \beta}$ with lower Willmore energy than $u$, having at most as many critical points as $u$ and such that $-\beta \leq w^{\prime}(x) \leq 0$ in $[0,1]$. We consider the function $\varphi$ defined in $[0,1]$ by

$$
\varphi(x):=x+w(x) w^{\prime}(x) .
$$

Note that $\varphi(0)=0$ and $\varphi(1)<0$. If $\varphi \leq 0$ in $[0,1]$ then the claim follows with $w=v$. Otherwise, there exists $x_{0} \in(0,1)$ such that $\varphi\left(x_{0}\right)=0$ and $\varphi \not \leq 0$ in a left neighbourhood of $x_{0}$. Then, the normal line at $\left(x_{0}, w\left(x_{0}\right)\right)$ to the graph of $w$ passes through the origin and we can substitute $w$ over $\left[-x_{0}, x_{0}\right]$ by a geodesic circular arc lowering the hyperbolic Willmore energy. This new function yields the claim. Notice that with this construction, due to the concavity of circles, the property $-\beta \leq w^{\prime} \leq 0$ is preserved and that we do not add critical points.

The following proposition summarises how by making use of Lemmas 3.8 and 3.9 we may pass to minimising sequences satisfying suitable a priori bounds.

Proposition 3.10. Let $\left(u_{k}\right)_{k \in \mathbb{N}}$ be a minimising sequence for $M_{\alpha, \beta}$ in $N_{\alpha, \beta}$ such that each $u_{k}$ has only finitely many critical points. Then, there exists a minimising sequence $\left(v_{k}\right)_{k \in \mathbb{N}} \subset N_{\alpha, \beta}$ such that for all $k \in \mathbb{N}$ it holds: $v_{k}$ has at most as many critical points as $u_{k}, \mathcal{W}_{h}\left(v_{k}\right) \leq \mathcal{W}_{h}\left(u_{k}\right)$,

$$
\begin{aligned}
& 0 \geq x+v_{k}(x) v_{k}^{\prime}(x) \text { and }-\beta \leq v_{k}^{\prime}(x) \leq 0 \text { for all } x \in[0,1] \\
& \text { and } \sqrt{1+\alpha^{2}-x^{2}} \leq v_{k}(x) \leq \sqrt{(\alpha+\beta)^{2}-x^{2}} \text { for all } x \in[-1,1] .
\end{aligned}
$$

\subsubsection{Proof of the existence theorem}

The proof of the following theorem follows the lines of the proof of [DDG, Theorem 3.9].

Theorem 3.11 (Existence and regularity). For each $\alpha>0$ and $\beta$ such that $\alpha \beta>1$ there exists a function $u \in C^{\infty}([-1,1],(0, \infty))$ such that the corresponding surface of revolution $\Gamma \subset \mathbb{R}^{3}$ solves the Dirichlet problem (1.4). This solution is positive and symmetric, and it has the following properties:

$$
\begin{aligned}
& -\beta \leq u^{\prime}(x)<0 \text { and } x+u(x) u^{\prime}(x)<0 \text { in }(0,1] \\
& \text { as well as } \sqrt{1+\alpha^{2}-x^{2}} \leq u(x) \leq \sqrt{(\alpha+\beta)^{2}-x^{2}} \quad \text { in }[-1,1] \text {. }
\end{aligned}
$$

Proof. Let $\left(u_{k}\right)_{k \in \mathbb{N}} \in N_{\alpha, \beta}$ be a minimising sequence for $M_{\alpha, \beta}$ such that $\mathcal{W}_{h}\left(u_{k}\right) \leq M_{\alpha, \beta}+1$ for all $k \in \mathbb{N}$. By the density of polynomials in $H^{2}(-1,1)$ and Proposition 3.10 we may assume that each element $u_{k}$ of the minimising sequence satisfies (3.1). We can estimate the Willmore energy from below as follows:

$$
\begin{aligned}
\mathcal{W}_{h}\left(u_{k}\right) & =\int_{-1}^{1} \frac{u_{k}^{\prime \prime}(x)^{2} u_{k}(x)}{\left(1+u_{k}^{\prime}(x)^{2}\right)^{\frac{5}{2}}} d x+2 \int_{-1}^{1} \frac{u_{k}^{\prime \prime}(x)}{\left(1+u_{k}^{\prime}(x)^{2}\right)^{\frac{3}{2}}} d x+\int_{-1}^{1} \frac{1}{u_{k}(x) \sqrt{1+u_{k}^{\prime}(x)^{2}}} d x \\
& \geq \frac{\alpha}{\left(1+\beta^{2}\right)^{\frac{5}{2}}} \int_{-1}^{1} u_{k}^{\prime \prime}(x)^{2} d x-\frac{4 \beta}{\sqrt{1+\beta^{2}}} .
\end{aligned}
$$


Thus, $\left(u_{k}\right)_{k \in \mathbb{N}}$ is uniformly bounded in $H^{2}(-1,1)$, and, eventually, after passing to a subsequence, Rellich's embedding theorem ensures the existence of $u \in H^{2}(-1,1)$ such that

$$
u_{k} \rightarrow u \quad \text { in } H^{2}(-1,1) \quad \text { and } \quad u_{k} \rightarrow u \in C^{1}([-1,1],(0, \infty)) .
$$

Making use of the strong convergence in $C^{1}([-1,1])$ and the weak convergence in $H^{2}(-1,1)$ of the sequence $\left(u_{k}\right)_{k \in \mathbb{N}}$, we have

$$
\begin{aligned}
M_{\alpha, \beta}+o(1) & =\mathcal{W}_{h}\left(u_{k}\right)=\int_{-1}^{1} \frac{u_{k}^{\prime \prime 2} u}{\left(1+u^{\prime 2}\right)^{\frac{5}{2}}} d x+\int_{-1}^{1} \frac{1}{u \sqrt{1+u^{\prime 2}}} d x-\frac{4 \beta}{\sqrt{1+\beta^{2}}}+o(1) \\
& \geq \int_{-1}^{1} \frac{u^{\prime 2} u}{\left(1+u^{\prime 2}\right)^{\frac{5}{2}}} d x+\int_{-1}^{1} \frac{1}{u \sqrt{1+u^{\prime 2}}} d x-\frac{4 \beta}{\sqrt{1+\beta^{2}}}+o(1)=\mathcal{W}_{h}(u)+o(1) .
\end{aligned}
$$

Thus, $u$ minimises $\mathcal{W}_{h}$ in the class of all positive and symmetric $H^{2}(-1,1)$-functions $v$ satisfying $v( \pm 1)=\alpha, v^{\prime}(+1)=-\beta$, and, therefore, $u$ weakly solves (2.5). Moreover, since the elements of the minimising sequence satisfy (3.1) then $u$ satisfies $x+u(x) u^{\prime}(x) \leq 0$ and $-\beta \leq u^{\prime}(x) \leq 0$ in $(0,1]$. From the first inequality it follows that $u^{\prime}<0$ in $(0,1]$.

The proof of smoothness of the solution is exactly as in [DDG, Theorem 3.9, Step 2].

Finally we show that $u$ satisfies $x+u(x) u^{\prime}(x)<0$ in $(0,1]$. Indeed, if $x_{0}+u\left(x_{0}\right) u^{\prime}\left(x_{0}\right)=0$ for some $x_{0} \in(0,1]$ then reasoning as in Lemma 3.9 and using that $M_{\alpha, \beta}=\mathcal{W}_{h}(u)$, we see that $u$ equals an arc of a geodesic circle in $\left[-x_{0}, x_{0}\right]$. But $u$ being a solution of $(2.5)$ implies by uniqueness of the initial value problem that $u$ is a geodesic circular arc on $[-1,1]$. But such an arc cannot satisfy the boundary conditions when $\alpha \beta>1$.

In Lemma 6.3 we prove further that $u^{\prime}$ is a decreasing function in $[0,1]$.

Proposition 3.12. Let $\alpha \beta>1$. Then, $M_{\widetilde{\alpha}, \beta}>M_{\alpha, \beta}$ for all $\widetilde{\alpha}$ such that $\widetilde{\alpha}>\alpha$.

Proof. Let $u_{\widetilde{\alpha}}$ be a solution of (1.4) for boundary values $\widetilde{\alpha}$ and $\beta$ as constructed in Theorem 3.11. By proceeding as in Lemma 3.5, i.e. inserting an appropriately chosen circular arc, we get a function $v \in N_{\alpha, \beta}$ such that $\mathcal{W}_{h}(v) \leq \mathcal{W}_{h}\left(u_{\tilde{\alpha}}\right)$. We prove that this inequality is in fact strict. As we have seen in the proof of Theorem 3.11, $u_{\widetilde{\alpha}}$ cannot be equal to an arc of a geodesic circle in an interval. Hence, by introducing a piece of a geodesic circle the energy strictly decreases. The claim follows since $\mathcal{W}_{h}(v) \geq M_{\alpha, \beta}$. Notice that, for the same reason, also this last inequality is strict.

\subsection{The case $\alpha \beta<1$}

The method of proof is related to that for the case $\alpha \beta>1$ but much simpler. The results are, in some sense, dual. For the monotonicity of the energy in the case $\alpha \beta>1$, we have constructed a function with lower Willmore energy and defined in a bigger interval. Now, with the same construction, the function is defined in a shorter interval. Moreover, we show that in this case we can confine ourselves to functions satisfying $x+u(x) u^{\prime}(x) \geq 0$ in $(0,1]$. A lower bound for the derivative follows directly from this inequality. We proceed quite similarly as in the case $\beta=0$, which was discussed in the previous paper [DDG] and which is included here.

\subsubsection{Monotonicity of the optimal energy}

In this case the Willmore energy is decreasing in $\alpha$. The proof is as in paragraph 3.2.1. For the sake of conciseness we formulate only the results. 
Lemma 3.13. Assume that $u \in N_{\alpha, \beta}$ has only finitely many critical points. Then, for each $\varrho \in[\alpha \beta, 1]$, there exist a positive and symmetric function $u_{\varrho} \in C^{1,1}([-\varrho, \varrho],(0, \infty))$ such that $u_{\varrho}(\varrho)=\alpha, u_{\varrho}^{\prime}(\varrho)=-\beta, u_{\varrho}^{\prime}(x) \leq 0$ for all $x \in[0, \varrho], u_{\varrho}$ has at most as many critical points as $u$, and, furthermore, one has

$$
\int_{-\varrho}^{\varrho} \kappa_{h}\left[u_{\varrho}\right]^{2} d s_{h}\left[u_{\varrho}\right] \leq \mathcal{W}_{h}(u) \text {. }
$$

In the next two results, for $\beta=0$ we interpret $1 / \beta$ as $\infty$.

Lemma 3.14. For each $u \in N_{\alpha, \beta}$ having only finitely many critical points and for each $\gamma \in\left[\alpha, \beta^{-1}\right]$ there exists a symmetric function $v \in C^{1,1}([-1,1],(0, \infty))$ having at most as many critical points as $u$ and satisfying: $v( \pm 1)=\gamma, v^{\prime}(1)=-\beta, v^{\prime}(x) \leq 0$ in $[0,1]$ and $\mathcal{W}_{h}(v) \leq \mathcal{W}_{h}(u)$.

Proposition 3.15. It holds that $M_{\widetilde{\alpha}, \beta} \geq M_{\alpha, \beta}$ for all $\alpha, \widetilde{\alpha}$ such that $0<\widetilde{\alpha} \leq \alpha \leq \frac{1}{\beta}$.

\subsubsection{Properties of minimising sequences}

In the next lemma we show that we can restrict ourselves to functions which are decreasing in $(0,1]$ and satisfy $x+u(x) u^{\prime}(x) \geq 0$ in $(0,1]$. A priori bounds follow directly from these observations.

Lemma 3.16. For each $u \in N_{\alpha, \beta}$ with only finitely many critical points there exists $v \in N_{\alpha, \beta}$ with lower Willmore energy than $u$, having at most as many critical points as $u$ and such that

$$
0 \leq x+v(x) v^{\prime}(x) \text { and } v^{\prime}(x) \leq 0 \text { for all } x \in[0,1] .
$$

Proof. By Lemma 3.2 and the following remark there exists $w \in N_{\alpha, \beta}$ with lower Willmore energy than $u$, having at most as many critical points as $u$ and such that $w^{\prime}(x) \leq 0$ in $[0,1]$. Let us consider the function $\varphi$ defined in $[0,1]$ by $\varphi(x):=x+w(x) w^{\prime}(x)$. Note that $\varphi(0)=0$ and $\varphi(1)>0$. If $\varphi \geq 0$ in $[0,1]$ then the claim follows with $w=v$. Otherwise, there exists $x_{0} \in(0,1)$ such that $\varphi\left(x_{0}\right)=0$ and $\varphi \geq 0$ in $\left(x_{0}, 1\right]$. Then, the normal line at $\left(x_{0}, w\left(x_{0}\right)\right)$ to the graph of $w$ passes through the origin and we can substitute $w$ over $\left[-x_{0}, x_{0}\right]$ by a geodesic circular arc lowering the hyperbolic Willmore energy. The new function so obtained yields the claim. With this construction we do not add critical points.

The following proposition characterises suitably modified minimising sequences.

Proposition 3.17. Let $\left(u_{k}\right)_{k \in \mathbb{N}}$ be a minimising sequence for $M_{\alpha, \beta}$ in $N_{\alpha, \beta}$ such that each $u_{k}$ has only finitely many critical points. Then, there exists a minimising sequence $\left(v_{k}\right)_{k \in \mathbb{N}} \subset N_{\alpha, \beta}$ such that for all $k \in \mathbb{N}: v_{k}$ has at most as many critical points as $u_{k}, \mathcal{W}_{h}\left(v_{k}\right) \leq \mathcal{W}_{h}\left(u_{k}\right)$ and satisfying:

$$
\begin{gathered}
0 \leq x+v_{k}(x) v_{k}^{\prime}(x), v_{k}^{\prime}(x) \leq 0 \text { and } v_{k}^{\prime}(x) \geq-\frac{x}{v_{k}(x)} \geq-\frac{x}{\alpha} \text { for all } x \in[0,1] \\
\text { and } \alpha \leq v_{k}(x) \leq \sqrt{1+\alpha^{2}-x^{2}} \text { for all } x \in[-1,1]
\end{gathered}
$$

\subsubsection{Proof of the existence theorem}

Thanks to Proposition 3.17 we prove now existence of a solution. The following result is a direct generalisation of [DDG, Theorem 1.1]. Its proof appears to be relatively simple but one should observe that via Lemma 3.2 the main constructions of [DDG, Theorem 3.8] are essentially used.

Theorem 3.18 (Existence and regularity). For each $\alpha>0$ and each $\beta \geq 0$ such that $\alpha \beta<1$ there exists a function $u \in C^{\infty}([-1,1],(0, \infty))$ such that the corresponding surface of revolution 
$\Gamma \subset \mathbb{R}^{3}$ solves the Dirichlet problem (1.4). This solution is positive and symmetric, and it has the following properties:

$$
-\frac{x}{\alpha} \leq u^{\prime}(x) \leq 0 \text { and } x+u(x) u^{\prime}(x)>0 \text { in }(0,1], \text { and } \alpha \leq u(x) \leq \sqrt{1+\alpha^{2}-x^{2}} \text { in }[-1,1] .
$$

Proof. Let $\left(u_{k}\right)_{k \in \mathbb{N}} \in N_{\alpha, \beta}$ be a minimising sequence for $M_{\alpha, \beta}$ such that $\mathcal{W}_{h}\left(u_{k}\right) \leq M_{\alpha, \beta}+1$ for all $k \in \mathbb{N}$. By Proposition 3.17 and the density of polynomials in $H^{2}(-1,1)$ we may assume that each element $u_{k}$ of the minimising sequence satisfies (3.3). The rest of the proof is on the same line as that of Theorem 3.11.

Proceeding as in the proof of Proposition 3.12, one can show that the energy is strictly decreasing.

Proposition 3.19. Let $\alpha>0, \beta \geq 0$ and $\alpha \beta<1$. Then, $M_{\widetilde{\alpha}, \beta}>M_{\alpha, \beta}$ for all $\widetilde{\alpha} \in(0, \alpha)$.

Also in this case, we can prove an additional qualitative information on our solution of (1.4) constructed in Theorem 3.18, namely that $u^{\prime}<0$ in $(0,1)$. This property is expected but here, it is slightly more involved to prove it when compared with the dual case $\alpha \beta>1$. It will prove to be helpful also for the constructions in the case $\beta<0$.

Lemma 3.20. Let $u$ be a solution of (1.4) minimising the hyperbolic Willmore energy in $N_{\alpha, \beta}$ as constructed in the proof of Theorem 3.18. Then, u satisfies $u^{\prime}<0$ in $(0,1)$.

Proof. We assume by contradiction that there exists $x_{0} \in(0,1)$ such that $u^{\prime}\left(x_{0}\right)=0$. This zero of $u^{\prime}$ is isolated because otherwise, by reflection and uniqueness for the initial value problem for (2.5), $u$ were even about $x_{0}$. In view of $u^{\prime} \leq 0$ on $[0,1]$ this would imply that $u^{\prime}(x)=0$ for $x$ close to $x_{0}$. This, however, is impossible since constants do not solve $(2.5)$.

Then there exist $a, b \in(0,1)$ such that $a<x_{0}<b, u^{\prime}(a)=u^{\prime}(b), u^{\prime}(x)>u^{\prime}(a)$ for all $x \in(a, b)$. Finally, by choosing $a, b$ close enough to $x_{0}$ and $\left|u^{\prime}(a)\right|$ small enough we may achieve that $\left(u(b)+u^{\prime}(b)(a-b)\right)\left(-u^{\prime}(b)\right) \leq a$ which will be used to insert a piece of a solution according to Theorem 3.18 on $[-a, a]$.

We construct a function $v \in N_{\alpha, \beta}$ with lower Willmore energy than $u$ and with non-zero derivative in $x_{0}$ as follows. $\left.v\right|_{[b, 1]}$ is equal to $\left.u\right|_{[b, 1]}$. Then $\left.v\right|_{[a, b)}$ equals the line starting at $(b, u(b))$ with derivative $u^{\prime}(b)$ and ending at $\left(a, u(b)+u^{\prime}(b)(a-b)\right)$. It remains to define $v$ on $[0, a)$. Here $v$ equals a solution of (1.4) in the interval $[-a, a]$ with boundary values $w( \pm a)=v(a)$ and $w^{\prime}(a)=u^{\prime}(a)$ obtained by a rescaled version of Theorem 3.18. Here we use that, by construction, $v(a)\left(-u^{\prime}(a)\right) \leq a$. See Figure 3 .

It remains to show that $v$ has strictly lower Willmore energy than $u$. We first compare the energies in $[-a, a]$. Since $v(a)>u(a)$ and $v^{\prime}(a)=u^{\prime}(a)$ by a rescaled version of Proposition 3.19 we see that the Willmore energy of $\left.v\right|_{[-a, a]}$ is strictly lower than the Willmore energy of $\left.u\right|_{[-a, a]}$. Now we compare the energies in $[a, b]$. From the definition of $v$ and since $u^{\prime}(a)=u^{\prime}(b)$ we have

$$
\begin{aligned}
& 2 \int_{a}^{b} \kappa_{h}[u]^{2} d s[u]-2 \int_{a}^{b} \kappa_{h}[v]^{2} d s[v] \\
= & 2 \int_{a}^{b} \frac{u^{\prime \prime 2} u}{\left(1+u^{\prime 2}\right)^{\frac{5}{2}}} d x+2 \int_{a}^{b} \frac{1}{u \sqrt{1+u^{\prime 2}}} d x+4 \int_{a}^{b} \frac{u^{\prime \prime}}{\left(1+u^{\prime 2}\right)^{\frac{3}{2}}} d x-2 \int_{a}^{b} \frac{1}{v \sqrt{1+v^{\prime 2}}} d x \\
\geq & 2 \int_{a}^{b} \frac{1}{u \sqrt{1+u^{\prime 2}}} d x-2 \int_{a}^{b} \frac{1}{v \sqrt{1+v^{\prime 2}}} d x \geq 0
\end{aligned}
$$


where in the last step we used that $v(x) \geq u(x)$ in $[-1,1]$ and $\left|v^{\prime}\right| \geq\left|u^{\prime}\right|$ in $[a, b]$. Comparing the total Willmore energies we then have $\mathcal{W}_{h}(u)>\mathcal{W}_{h}(v)$. A contradiction since $u$ is the minimiser for $M_{\alpha, \beta}$ in $N_{\alpha, \beta}$.

a)

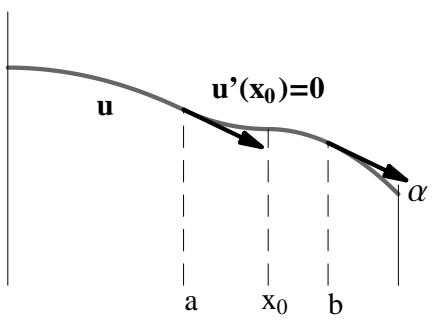

b)

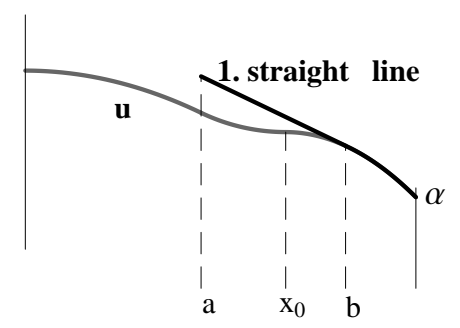

c)

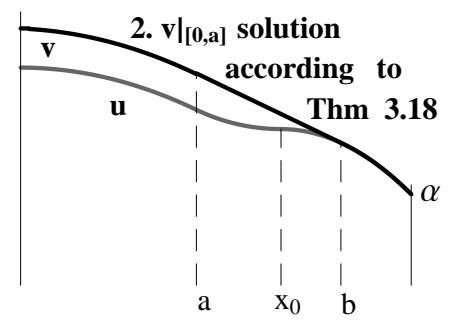

Figure 3: Proof of Lemma 3.20.

\section{Existence result: The case $\beta<0$}

In this section we consider $\beta<0$ fixed, while $\alpha$ varies in the positive real numbers.

The case $\beta<0$ is quite different from $\beta \geq 0$. In the latter, our constructions were based on inserting parts of geodesic circles. Here, also catenoids will play an important role. Each of these minimal surfaces is generated by the graph of $u(x):=\cosh (b x) / b, x \in[-1,1]$, for some $b>0$. They are solutions of (1.4) for particular values of $\alpha$ and $\beta$. Given $\beta<0$ we denote

$$
\alpha_{\beta}:=\frac{\cosh (b)}{b} \text { with } b=\operatorname{arsinh}(-\beta) .
$$

Notice that $\alpha_{\beta} \operatorname{arsinh}(-\beta)=\sqrt{1+\beta^{2}}$. We comment on these particular solutions in some more detail in the next subsection. Then, the cases $\alpha>\alpha_{\beta}$ and $\alpha<\alpha_{\beta}$ have to be treated separately. In the first case the energy is increasing for $\alpha$ increasing, while in the second case it is decreasing for $\alpha$ increasing. Moreover, the behaviour of the solution we construct is different in the two cases. If $\alpha>\alpha_{\beta}$ the solution satisfies $u^{\prime}>0$ in $(0,1]$ while for $\alpha<\alpha_{\beta}$ a further critical point could in principle appear in $(0,1)$. An intuition for this is given by looking for a function of the kind $v(x)=\cosh (\lambda(x-d)) / \lambda$ choosing $\lambda$ and $d$ suitably such that $v$ satisfies $v(1)=\alpha$ and $v^{\prime}(1)=-\beta$. If $\alpha>\alpha_{\beta}$, then $d<0$. This tell us that, in some sense, there is not enough space for a catenoid. On the other hand, if $\alpha<\alpha_{\beta}$, then $d>0$ so there is too much space for a catenoid. One could think that a further critical point should show up in $(0,1)$ together with a solution for $\beta=0$ in the inner part. By Lemma 5.2 this will certainly happen for $\alpha$ close enough to 0. However, we are not able to determine the precise range of $\alpha \in\left(0, \alpha_{\beta}\right)$ where this extra local minimum may be observed. The function $u_{C l}(x)=2-\sqrt{2-x^{2}}$ for $x \in[-1,1]$ (part of the - projected - Clifford torus) solves the Willmore equation (1.4) for $\alpha=1$ and $\beta=-1\left(\alpha<\alpha_{\beta}\right)$ and has no critical point in $(0,1)$.

\subsection{The case $\alpha=\alpha_{\beta}$ : The catenoid}

We summarise the main properties of the catenoids as explicit minimal surface solutions.

Lemma 4.1. For $\beta<0$ and $\alpha$ such that $\alpha=\cosh (b) / b$ with $b=\operatorname{arsinh}(-\beta)$, the part of the catenoid $\Gamma \subset \mathbb{R}^{3}$ generated by the function $u(x)=\cosh (b x) / b, x \in[-1,1]$, solves the Dirichlet problem (1.4). 
Moreover, the corresponding surface of revolution is the minimiser of the Willmore functional (1.1) among all axially symmetric surfaces generated by graphs of symmetric positive functions in $C^{1,1}([-1,1],(0, \infty))$ with $v( \pm 1)=\alpha$ and $v^{\prime}(1)=-\beta$.

Proof. Rotating the graph of $u$ around the $x$-axis generates a minimal surface, i.e. a surface such that $H \equiv 0$. Moreover, by the choice of $b$ the function $u$ satisfies the boundary conditions $u( \pm 1)=\alpha_{\beta}$ and $u^{\prime}(1)=-\beta$. For any $v \in N_{\alpha, \beta}$ we have

$$
\begin{aligned}
\mathcal{W}_{h}(v) & =2 \int_{0}^{1}\left(\frac{v^{\prime \prime}}{\left(1+v^{\prime 2}\right)^{\frac{3}{2}}}-\frac{1}{v(x) \sqrt{1+v^{\prime}(x)^{2}}}\right)^{2} v(x) \sqrt{1+v^{\prime}(x)^{2}} d x+8 \int_{0}^{1} \frac{v^{\prime \prime}}{\left(1+v^{\prime 2}\right)^{\frac{3}{2}}} d x \\
& \geq-8 \frac{\beta}{\sqrt{1+\beta^{2}}}=\mathcal{W}_{h}(u) .
\end{aligned}
$$

This shows that $u$ minimises $M_{\alpha, \beta}$ and, by Lemma 2.1 , that the axially symmetric surface generated by $u$ minimises the Willmore functional among axially symmetric surfaces generated by graphs of symmetric functions satisfying the prescribed boundary conditions.

Remark 4.2. Notice that given $\beta<0$, there exist unique associated $b$ and $\alpha_{\beta}$ defined as in (4.1). When $\beta$ varies in the negative real numbers, $\alpha_{\beta}$ is bounded from below. Indeed, the function $b \mapsto \frac{1}{b} \cosh (b)$ has precisely one minimum at $b^{*}>0$ which is the solution of the equation

$$
\cosh \left(b^{*}\right)=b^{*} \sinh \left(b^{*}\right), \quad b^{*}=1.1996786 \ldots
$$

The value $\alpha^{*}$ defined in (1.5) denotes the minimal value of $(0, \infty) \ni b \mapsto \cosh (b) / b$. For $\alpha<\alpha^{*}$, there are no minimal surfaces of revolution solving (1.4). If $\alpha=\alpha^{*}$, then $\frac{\cosh \left(b^{*} x\right)}{b^{*}}$ is a minimal surface solution for the boundary datum $\beta=-\sinh \left(b^{*}\right)$. In the case $\alpha>\alpha^{*}$, there are two positive real numbers $b_{1}(\alpha), b_{2}(\alpha)$ such that

$$
b_{1}(\alpha)<b^{*}<b_{2}(\alpha) \quad \text { and } \quad \frac{\cosh \left(b_{1}\right)}{b_{1}}=\alpha=\frac{\cosh \left(b_{2}\right)}{b_{2}} .
$$

Two different minimal surfaces with the same height $\alpha$ in 1 and different boundary slopes correspond to these two values. These two catenoids play an important role in what follows.

\subsection{The case $\alpha>\alpha_{\beta}$}

In this case the height prescribed at the boundary is bigger than the height of the catenoid centered at 0 and having derivative $-\beta$ at $x=1$. As observed in Remark 4.2, there are two catenoids that in 1 have the height $\alpha$. These are $\cosh \left(b_{1} x\right) / b_{1}$ and $\cosh \left(b_{2} x\right) / b_{2}$ with $b_{1}=b_{1}(\alpha)$ and $b_{2}=b_{2}(\alpha)$ defined in (4.3). Since $\alpha=\cosh \left(b_{1}\right) / b_{1}=\cosh \left(b_{2}\right) / b_{2}>\alpha_{\beta}=\cosh (b) / b$, it follows that $b_{1}<b<b_{2}$ and $\sinh \left(b_{1}\right)<-\beta=\sinh (b)<\sinh \left(b_{2}\right)$. So, close to $x=1$, the graph of $u \in N_{\alpha, \beta}$ is between the graphs of the two catenoids (see Figure 4 ). In the following we use this observation to characterise functions in $N_{\alpha, \beta}$ with low Willmore energy.

We explain first how to lower the Willmore energy by inserting $C^{1,1}$-smoothly suitable parts of catenoids. This construction also yields that we may restrict ourselves to functions increasing in $[0,1]$. To proceed we have to distinguish between $\alpha \leq-\beta$ and $\alpha>-\beta$. The line $y \mapsto \alpha y$ is crucial for rescaling and the different positions of curves in $N_{\alpha, \beta}$ relative to this line close to $x=1$ require different geometric constructions. If $-\beta \geq \alpha$, these constructions allow for suitably modifying minimising sequences so that strong enough a priori bounds are available. If $-\beta<\alpha$, we need to pass to a smaller class of admissible functions instead of $N_{\alpha, \beta}$ in order to avoid a possible loss of compactness. 

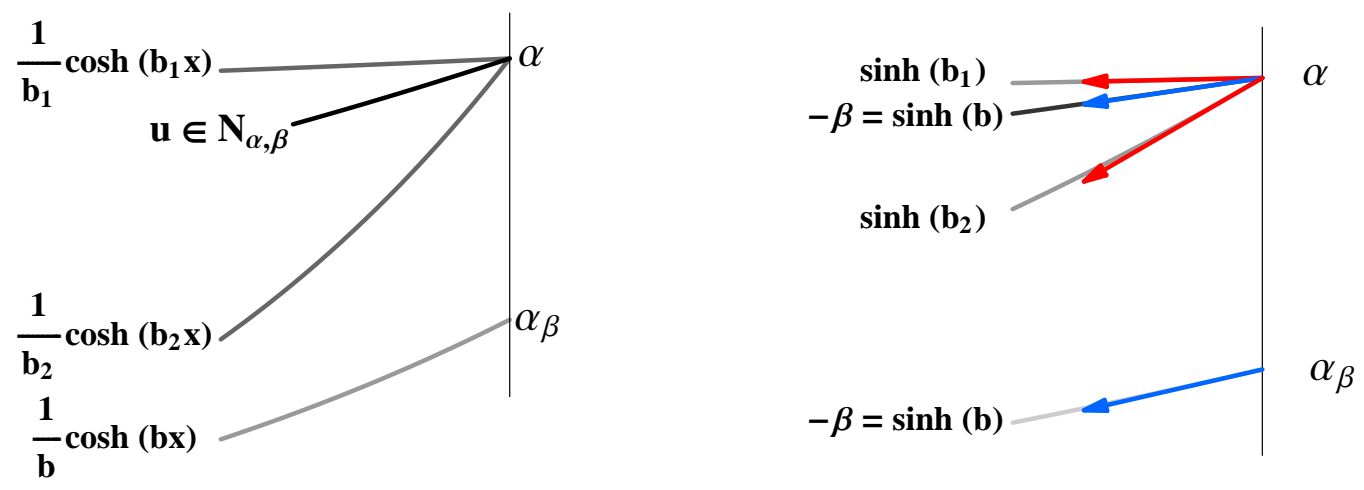

Figure 4: Comparison between $u \in N_{\alpha, \beta}$ and the catenoids $\cosh \left(b_{1} x\right) / b_{1}$ and $\cosh \left(b_{2} x\right) / b_{2}$.

\subsubsection{First observations}

In this subsection we introduce some geometric constructions which lower the Willmore energy and will be used repeatedly in the rest of this section. In the next lemma we formulate a criterion which allows for inserting a piece of a catenoid in a $C^{1,1}$-smooth way. This criterion is dual to the condition $0=x_{0}+v\left(x_{0}\right) v^{\prime}\left(x_{0}\right)$ which allows for inserting $C^{1,1}$-smoothly a part of a geodesic circle on $\left[-x_{0}, x_{0}\right]$.

Lemma 4.3. Fix $a>0$. Let $f \in C^{1,1}([0, a],(0,+\infty))$ be such that $f^{\prime}(0)=0$. Furthermore assume that there is $x_{0} \in(0, a)$ such that $f^{\prime}\left(x_{0}\right)>0$ and

$$
1-\frac{1}{\sqrt{1+f^{\prime}\left(x_{0}\right)^{2}}} \cosh \left(\frac{\sqrt{1+f^{\prime}\left(x_{0}\right)^{2}}}{f\left(x_{0}\right)} x_{0}\right)=0 .
$$

Then, the function

$$
v(x):=\left\{\begin{array}{ll}
\frac{1}{\gamma} \cosh (\gamma x) & \text { for } x \in\left[0, x_{0}\right] \\
f(x) & \text { for } x \in\left[x_{0}, a\right]
\end{array} \quad \text { with } \gamma:=\frac{\sqrt{1+f^{\prime}\left(x_{0}\right)^{2}}}{f\left(x_{0}\right)}\right.
$$

is in $C^{1,1}([0, a],(0,+\infty))$, and it satisfies $v^{\prime}(0)=0$.

Proof. It is sufficient to study the behaviour in $x_{0}$. We see that

$$
\lim _{x \nearrow x_{0}} v(x)=\frac{f\left(x_{0}\right)}{\sqrt{1+f^{\prime}\left(x_{0}\right)^{2}}} \cosh \left(\frac{\sqrt{1+f^{\prime}\left(x_{0}\right)^{2}}}{f\left(x_{0}\right)} x_{0}\right)=f\left(x_{0}\right),
$$

using (4.4). For the derivative we find

$$
\lim _{x \nearrow x_{0}} v^{\prime}(x)=\sinh \left(\frac{\sqrt{1+f^{\prime}\left(x_{0}\right)^{2}}}{f\left(x_{0}\right)} x_{0}\right)=\sqrt{\cosh ^{2}\left(\frac{\sqrt{1+f^{\prime}\left(x_{0}\right)^{2}}}{f\left(x_{0}\right)} x_{0}\right)-1}=f^{\prime}\left(x_{0}\right),
$$

using (4.4) again and the fact that $f^{\prime}\left(x_{0}\right)>0$.

Remark 4.4. Notice that, by the convexity of cosh, if $f^{\prime}(x) \leq \delta$ for all $x \in[0, a]$ then also $v^{\prime}(x) \leq \delta$ for all $x \in[0, a]$. 
In the case $\beta \geq 0$ we could without loss of generality consider only functions satisfying $x+$ $u(x) u^{\prime}(x) \geq 0$ (or $\leq 0$ ). In the next lemma we deduce a dual condition in the case $\beta<0$ and $\alpha>\alpha_{\beta}$. Here we use that an arc of catenoid gives the lowest Willmore energy when connecting one point with prescribed positive derivative to another with prescribed and bigger positive derivative. As a consequence we see that without loss of generality it is sufficient to consider functions $u \in N_{\alpha, \beta}$ such that $u^{\prime}>0$ in $(0,1]$.

Lemma 4.5. For each $u \in N_{\alpha, \beta}$ there exists $v \in N_{\alpha, \beta}$ such that $\mathcal{W}_{h}(v) \leq \mathcal{W}_{h}(u), v^{\prime}>0$ in $(0,1]$ and $v$ satisfies

$$
1-\frac{1}{\sqrt{1+v^{\prime}(x)^{2}}} \cosh \left(\frac{\sqrt{1+v^{\prime}(x)^{2}}}{v(x)} x\right) \geq 0 \text { in }[0,1] .
$$

Proof. Let $u \in N_{\alpha, \beta}$ be arbitrary. For easy reference we here denote by $g(x)$ the function on the left hand side of inequality (4.5) with $v$ replaced by $u$. Obviously, $g(0)=0$. Since $b=\operatorname{arsinh}(-\beta)$, we find in $x=1$ :

$$
\begin{aligned}
g(1) & =1-\frac{1}{\sqrt{1+\beta^{2}}} \cosh \left(\frac{\sqrt{1+\beta^{2}}}{\alpha}\right)=1-\frac{1}{\cosh (b)} \cosh \left(\frac{\cosh (b)}{\alpha}\right) \\
& =\frac{1}{\alpha_{\beta}}\left(\alpha_{\beta}-\frac{1}{b} \cosh \left(\frac{\alpha_{\beta}}{\alpha} b\right)\right)=\frac{1}{b \alpha_{\beta}}\left(\cosh (b)-\cosh \left(\frac{\alpha_{\beta}}{\alpha} b\right)\right)>0,
\end{aligned}
$$

using (4.1) and that $\alpha>\alpha_{\beta}$. If $g(x)$ is negative at some point in $(0,1)$, there exists a largest $x_{0} \in(0,1)$ such that $g\left(x_{0}\right)=0$ and $g(x)>0$ in $\left(x_{0}, 1\right]$. We first observe that $u^{\prime}\left(x_{0}\right)>0$. This follows from $u^{\prime}(1)>0$, the continuity of $u^{\prime}$ and the fact that $u^{\prime}(x) \neq 0$ for $x>0$ where $g(x) \geq 0$. Then by Lemma 4.3 with $a=1$ and $f=u$ we can define a new function $v$ that coincides with $u$ on $\left[x_{0}, 1\right]$ and with a cosh on $\left[0, x_{0}\right]$ (see Figure 5 , a)). Since $v^{\prime}(0)=0$ we may extend it by symmetry to a $C^{1,1}$-function on $[-1,1]$. For this new function (4.5) is always satisfied. Moreover $v$ has lower Willmore energy than $u$. Indeed, we have

$$
\begin{aligned}
\mathcal{W}_{h}(u)= & 2 \int_{x_{0}}^{1} \kappa_{h}[u]^{2} d s_{h}[u]+2 \int_{0}^{x_{0}}\left(\frac{u^{\prime \prime}(x)}{\left(1+u^{\prime}(x)^{2}\right)^{\frac{3}{2}}}-\frac{1}{u(x) \sqrt{1+u^{\prime}(x)^{2}}}\right)^{2} u(x) \sqrt{1+u^{\prime}(x)^{2}} d x \\
& +8 \int_{0}^{x_{0}} \frac{u^{\prime \prime}(x)}{\left(1+u^{\prime}(x)^{2}\right)^{\frac{3}{2}}} d x \geq 2 \int_{x_{0}}^{1} \kappa_{h}[u]^{2} d s_{h}[u]+8 \frac{u^{\prime}\left(x_{0}\right)}{\sqrt{1+u^{\prime}\left(x_{0}\right)^{2}}}=\mathcal{W}_{h}(v),
\end{aligned}
$$

by definition of $v$ and since $x \mapsto \cosh (b x) / b$ satisfies $H(x) \equiv 0$.

Finally, $v^{\prime}(x)>0$ in $(0,1)$ since $v$ satisfies $(4.5)$ in $[0,1]$ and $v^{\prime}(1)=-\beta>0$.

Remark 4.6. Notice that if $u^{\prime}(x) \leq \delta$ for all $x \in[0,1]$ then also $v^{\prime}(x) \leq \delta$ for all $x \in[0,1]$. This is due to the convexity of cosh.

In what follows we consider functions having the following property:

$$
u \text { satisfies: } 1-\frac{1}{\sqrt{1+u^{\prime}(x)^{2}}} \cosh \left(\frac{\sqrt{1+u^{\prime}(x)^{2}}}{u(x)} x\right) \geq 0 \text { in }[0,1] \text { and } u^{\prime}>0 \text { in }(0,1] \text {. }
$$

We first remark that this condition is scaling invariant, i.e. it is also satisfied for $u_{r}(x)=\frac{1}{r} u(r x)$, $x \in\left[-\frac{1}{r}, \frac{1}{r}\right]$. The fact that $u \in N_{\alpha, \beta}$ satisfies (4.6) gives us information on the behaviour of the 
a)

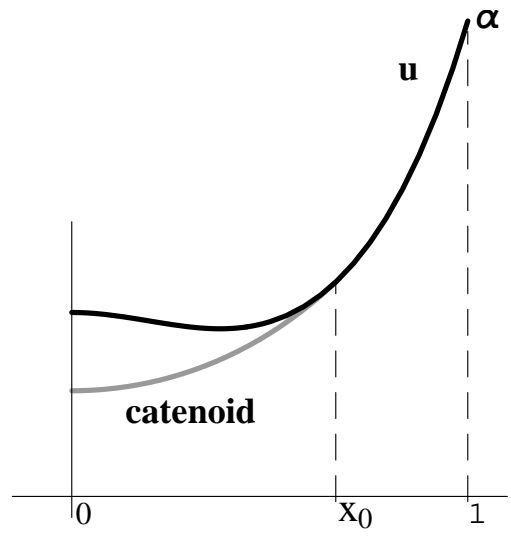

b)

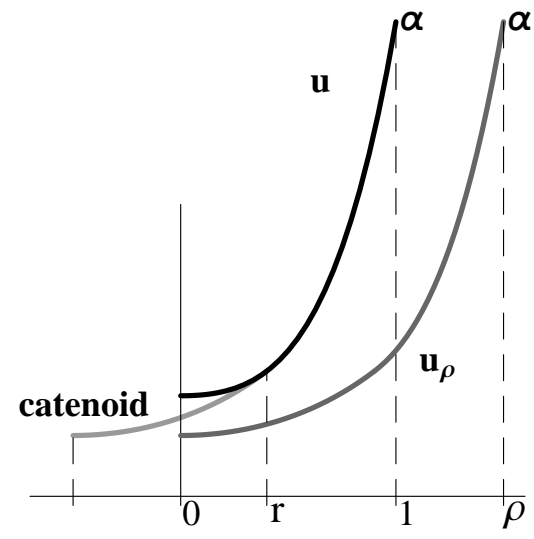

Figure 5: Proof of Lemma 4.5 (left) and of Lemma 4.11 (right).

graph of $u$ with respect to the two catenoids going through the point $(1, \alpha)$ and centered at the origin. We recall that these are the functions

$$
\frac{\cosh \left(b_{1} x\right)}{b_{1}} \text { and } \frac{\cosh \left(b_{2} x\right)}{b_{2}} \text { with } b_{1}<b^{*}<b_{2} \text { such that } \frac{\cosh \left(b_{1}\right)}{b_{1}}=\alpha=\frac{\cosh \left(b_{2}\right)}{b_{2}} .
$$

Here $b^{*}$ is the unique solution of $\cosh \left(b^{*}\right)=b^{*} \sinh \left(b^{*}\right), b^{*}=1.1996786 \ldots$. We recall that also $b_{1}<b<b_{2}$.

One might expect that $x \mapsto \frac{1}{b_{1}} \cosh \left(b_{1} x\right)$ and $x \mapsto \frac{1}{b_{2}} \cosh \left(b_{2} x\right)$ could serve as comparison functions. Unfortunately this works out only partially.

Lemma 4.7. Let $u \in N_{\alpha, \beta}$ satisfy (4.6). For $x \in[0,1)$ we have:

$$
u(x)=\frac{1}{b_{1}} \cosh \left(b_{1} x\right) \Rightarrow u^{\prime}(x) \geq \sinh \left(b_{1} x\right) .
$$

More restrictively, if $x \in\left[b^{*} / b_{2}, 1\right]$, then

$$
u(x)=\frac{1}{b_{2}} \cosh \left(b_{2} x\right) \Rightarrow u^{\prime}(x) \leq \sinh \left(b_{2} x\right) .
$$

Proof. It is convenient to rewrite the inequality (4.6) by means of $\cosh (\operatorname{arsinh}(y))=\sqrt{1+y^{2}}$ as follows

$$
\operatorname{arsinh}\left(u^{\prime}(x)\right) \geq \cosh \left(\operatorname{arsinh}\left(u^{\prime}(x)\right)\right) \frac{x}{u(x)} \text { in }[0,1],
$$

or

$$
\frac{u(x)}{x} \geq \frac{\cosh \left(\operatorname{arsinh}\left(u^{\prime}(x)\right)\right)}{\operatorname{arsinh}\left(u^{\prime}(x)\right)} \text { in }(0,1],
$$

We already know - see Figure 4 - that in a left neighbourhood of 1 we have $\cosh \left(b_{1} x\right) / b_{1}>u(x)>$ $\cosh \left(b_{2} x\right) / b_{2}$. Let $x \in(0,1)$ be such that $u(x)=\cosh \left(b_{i} x\right) / b_{i}, i=1$ or 2 . Then from (4.6) it follows that

$$
\frac{\cosh \left(b_{i} x\right)}{b_{i} x} \geq \frac{\cosh \left(\operatorname{arsinh}\left(u^{\prime}(x)\right)\right)}{\operatorname{arsinh}\left(u^{\prime}(x)\right)} .
$$

We consider first $i=1$. Since $x \in(0,1), b_{1}<b^{*}$ which is the minimum of $g(b)=\frac{1}{b} \cosh (b)$, it follows that $b_{1} x$ is left from this minimum. Hence, an argument with a smaller $g$-value than $b_{1} x$ must be right from $b_{1} x$, i.e. $\operatorname{arsinh}\left(u^{\prime}(x)\right) \geq b_{1} x, u^{\prime}(x) \geq \sinh \left(b_{1} x\right)$.

For $i=2$ and $x \geq b^{*} / b_{2}$ we have that $b_{2} x$ is right of the $g$-minimum $b^{*}$. Smaller $g$-values than $g\left(b_{2} x\right)$ are attained at most left from $b_{2} x$, i.e. if $\operatorname{arsinh}\left(u^{\prime}(x)\right) \leq b_{2} x, u^{\prime}(x) \leq \sinh \left(b_{2} x\right)$. 
Remark 4.8. 1. If a function $u \in N_{\alpha, \beta}$ satisfying (4.6) intersects $x \mapsto \frac{1}{b_{1}} \cosh \left(b_{1} x\right)$ in a largest point $x_{0} \in(0,1)$, then left of $x_{0}$, it is below the cosh so that $u^{\prime}\left(x_{0}\right) \leq \sinh \left(b_{1} x_{0}\right)$. The previous lemma shows that, on the other hand, $u^{\prime}\left(x_{0}\right) \geq \sinh \left(b_{1} x_{0}\right)$ so that $u^{\prime}\left(x_{0}\right)=\sinh \left(b_{1} x_{0}\right)$. The function $u$ is in $x_{0}$ tangent to $x \mapsto \frac{1}{b_{1}} \cosh \left(b_{1} x\right)$ so that the latter may replace $C^{1,1}$-smoothly $\left.u\right|_{\left[-x_{0}, x_{0}\right]}$. This new function $v$ is again in $N_{\alpha, \beta}$, has lower Willmore energy, satisfies (4.6) and in addition

$$
v(x) \leq \frac{\cosh \left(b_{1} x\right)}{b_{1}} \text { in }[0,1] .
$$

2. Analogously we may achieve on minimising sequences that

$$
u(x) \geq \frac{\cosh \left(b_{2} x\right)}{b_{2}} \text { in }\left[b^{*} / b_{2}, 1\right] .
$$

Unfortunately there is no obvious mechanism to achieve a lower bound also on $\left[-b^{*} / b_{2}, b^{*} / b_{2}\right]$.

3. Analogously one may also achieve on minimising sequences that $v(x) \geq \alpha^{*}|x|$. Again, this bound is not sufficient in order to ensure compactness.

In order to prove strong enough lower bounds on suitable minimising sequences, we fist achieve uniform derivative bounds. Then, the following lemma will prove to be useful.

Lemma 4.9. Let $\alpha>0$ and $\beta<0$ be arbitrary and $u \in N_{\alpha, \beta}$ such that there exists $x_{0} \in[0,1)$ so that $u^{\prime}\left(x_{0}\right)=0, u^{\prime}>0$ in $\left(x_{0}, 1\right]$ and $u^{\prime} \leq 0$ in $\left[0, x_{0}\right]$. Let $\gamma:=\max _{x \in\left[x_{0}, 1\right]} u^{\prime}(x)$. Then it holds that

$$
\min _{x \in[0,1]} u(x)=u\left(x_{0}\right) \geq \gamma \frac{1-x_{0}}{e^{C}-1},
$$

where $C>0$ a constant depending monotonically on $\mathcal{W}_{h}(u), \gamma$ and $\beta$.

Proof. We estimate the Willmore energy from below as follows

$$
\begin{aligned}
\mathcal{W}_{h}(u) & \geq 2 \int_{x_{0}}^{1}\left(\frac{u^{\prime \prime} u}{\left(1+u^{\prime 2}\right)^{\frac{3}{2}}}+\frac{1}{\sqrt{1+u^{\prime}(x)^{2}}}\right)^{2} \frac{\sqrt{1+u^{\prime}(x)^{2}}}{u(x)} d x \\
& \geq 2 \int_{x_{0}}^{1} \frac{1}{u \sqrt{1+u^{\prime}(x)^{2}}} d x+4 \int_{x_{0}}^{1} \frac{u^{\prime \prime}}{\left(1+u^{\prime 2}\right)^{\frac{3}{2}}} d x \\
& =2 \int_{x_{0}}^{1} \frac{1}{u \sqrt{1+u^{\prime}(x)^{2}}} d x-4 \frac{\beta}{\sqrt{1+\beta^{2}}} .
\end{aligned}
$$

We recall here that from $(4.2)$ it follows that $\mathcal{W}_{h}(v) \geq-8 \beta / \sqrt{1+\beta^{2}}$ for all $v \in N_{\alpha, \beta}$. Since $u^{\prime} \leq \gamma$ and, hence, $u(x) \leq u\left(x_{0}\right)+\gamma\left(x-x_{0}\right)$ for $x \in\left[x_{0}, 1\right]$ we get

$$
\begin{aligned}
\mathcal{W}_{h}(u) & \geq \frac{2}{\sqrt{1+\gamma^{2}}} \int_{x_{0}}^{1} \frac{1}{u\left(x_{0}\right)+\gamma\left(x-x_{0}\right)} d x-4 \frac{\beta}{\sqrt{1+\beta^{2}}} \\
& =\frac{2}{\gamma \sqrt{1+\gamma^{2}}} \log \left(1+\frac{\gamma\left(1-x_{0}\right)}{u\left(x_{0}\right)}\right)-4 \frac{\beta}{\sqrt{1+\beta^{2}}}
\end{aligned}
$$

that gives

$$
1+\frac{\gamma\left(1-x_{0}\right)}{u\left(x_{0}\right)} \leq e^{C}
$$

with $C$ depending monotonically (increasing) on $\mathcal{W}_{h}(u), \beta$ and $\gamma$. The claim follows. 
Later we show by possibly inserting a rescaled solution with zero boundary slope that the assumption just made on $u \in N_{\alpha, \beta}$ is not restrictive.

In the following remark we collect some conclusions which can be drawn for bounds on the derivatives of functions satisfying (4.6).

Remark 4.10. Let $u \in N_{\alpha, \beta}$ satisfy (4.6) in $[0,1]$. Let $b_{1}=b_{1}(\alpha)$ and $b_{2}=b_{2}(\alpha)$ be as defined in (4.7). Then, one has the following inequalities:

1. $u^{\prime}(x)=-\beta$ implies $u(x) \geq \alpha_{\beta} x$ with $\alpha_{\beta}$ defined in (4.1);

2. $u^{\prime}(x)=\sinh \left(b_{i}\right), i=1,2$, implies $u(x) \geq \alpha x$;

3. $u(x) \leq \alpha x$ implies $\sinh \left(b_{1}\right) \leq u^{\prime}(x) \leq \sinh \left(b_{2}\right)$. (In general $u(x) \leq \gamma x, \gamma>\alpha^{*}$, implies bounds for the value of the derivative).

As explained at the beginning of this section we proceed now by discussing the cases $\alpha \leq-\beta$ and $\alpha>-\beta$ separately. This distinction requires to study the minimisation process in different classes of admissible functions.

\subsubsection{The case $-\beta \geq \alpha$}

We first prove that the energy is monotonically increasing in $\alpha$. Then, using that $u$ satisfies (4.6), we get a priori bounds on the derivative leading to an existence theorem.

\section{Monotonicity of the optimal energy}

For $\beta \geq 0$, when studying monotonicity of the energy we constructed new functions with lower Willmore energy by inserting a suitable arc of a circle. Here we do an analogous construction inserting arcs of catenoids.

Lemma 4.11. Assume that $u \in N_{\alpha, \beta}$ satisfies (4.6). Then for each $\varrho \in\left(1, \alpha / \alpha_{\beta}\right)$ there exists $u_{\varrho} \in C^{1,1}([-\varrho, \varrho],(0, \infty))$ positive, symmetric and such that $u_{\varrho}( \pm \varrho)=\alpha, u_{\varrho}^{\prime}(\varrho)=-\beta,(4.5)$ is satisfied in $[0, \varrho]$ and $u_{\varrho}^{\prime}>0$ in $(0, \varrho]$ as well as

$$
\int_{-\varrho}^{\varrho} \kappa_{h}\left[u_{\varrho}\right]^{2} d s_{h}\left[u_{\varrho}\right] \leq \mathcal{W}_{h}(u)
$$

Proof. The idea of the construction is to change the graph of $\left.u\right|_{[0, r]}$ for some $r>0$ by inserting $C^{1,1}$ smoothly an arc of a catenoid, see Figure $5, \mathrm{~b}$ ). Then, translating and extending it by symmetry we find an even function. Choosing $r$ appropriately depending on $\varrho$, we obtain a function defined in the bigger interval $[-\varrho, \varrho]$ and satisfying $(4.5)$ in $[0, \varrho]$. We give now the technical details of the construction.

For $\varrho \in\left(1, \alpha / \alpha_{\beta}\right)$ let $r \in(0,1)$ be the biggest element in $(0,1)$ such that $\varphi(r)=0$ where $\varphi$ is defined by

$$
\varphi(x)=x-1+\varrho-\operatorname{arsinh}\left(u^{\prime}(x)\right) \frac{u(x)}{\sqrt{1+u^{\prime}(x)^{2}}} \quad \text { for } \quad x \in[0,1] .
$$

Such an element exists since we have $\varphi(0)>0$ and, by recalling $\operatorname{arsinh}(-\beta) / \sqrt{1+\beta^{2}}=1 / \alpha_{\beta}$, that $\varphi(1)<0$. Let $\lambda$ be defined by $\lambda=\sqrt{1+u^{\prime}(r)^{2}} / u(r)$. Then the function $u_{\varrho}$ defined in $[0,1]$ by

$$
u_{\varrho}(x):= \begin{cases}u(x+1-\varrho) & \text { if } r-1+\varrho \leq x \leq \varrho, \\ \frac{1}{\lambda} \cosh (\lambda x) & \text { if } 0 \leq x<r-1+\varrho,\end{cases}
$$


and symmetrically extended to $[-1,1]$ is in $C^{1,1}([-1,1],(0, \infty))$. It satisfies $u_{\varrho}( \pm \varrho)=\alpha, u_{\varrho}^{\prime}(\varrho)=$ $-\beta, u_{\varrho}^{\prime}>0$ in $(0, \varrho]$ and has a smaller curvature integral than $u$.

It remains to prove that $u_{\varrho}$ satisfies $(4.5)$ in $[0, \varrho]$. For easy notation let consider $\psi$ defined by

$$
\psi(x)=\frac{1}{\sqrt{1+u_{\varrho}^{\prime}(x)^{2}}} \cosh \left(\frac{\sqrt{1+u_{\varrho}^{\prime}(x)^{2}}}{u_{\varrho}(x)} x\right) \quad \text { for } \quad x \in[0, \varrho] .
$$

We need to show that $\psi(x) \leq 1$ in $[0, \varrho]$. By construction, $\psi(x) \equiv 1$ in $[0, r-1+\varrho]$ and from $\sqrt{1+\beta^{2}} / \alpha_{\beta}=\operatorname{arsinh}(-\beta)$ we conclude that $\psi(\varrho)<1$. The claim follows since, by choice of $r$, the biggest point in $[0, \varrho]$ such that $\psi(x)=1$ is $x=r-1+\varrho$.

By rescaling we obtain:

Corollary 4.12. For each $u \in N_{\alpha, \beta}$ such that $u$ satisfies (4.6) and for each $\gamma \in\left[\alpha_{\beta}, \alpha\right.$ ) there exists a positive symmetric $v \in C^{1,1}([-1,1],(0, \infty))$ such that $v$ satisfies $(4.6), v( \pm 1)=\gamma, v^{\prime}(1)=-\beta$ and $\mathcal{W}_{h}(v) \leq \mathcal{W}_{h}(u)$.

Proof. If $\gamma=\alpha_{\beta}$ the claim follows choosing $v(x)=\cosh (b x) / b$ with $b=-\operatorname{arsinh}(-\beta)$. If $\gamma \in\left(\alpha_{\beta}, \alpha\right)$ the claim follows from Lemma 4.11 by rescaling.

Proposition 4.13. Let $M_{\alpha, \beta}$ be defined as in (2.7). Then for $\tilde{\alpha} \geq \alpha \geq \alpha_{\beta}$ we have $M_{\tilde{\alpha}, \beta} \geq M_{\alpha, \beta}$.

Proof. Let $\left(u_{k}\right)_{k \in \mathbb{N}}$ be a minimising sequence for $M_{\tilde{\alpha}, \beta}$ in $N_{\tilde{\alpha}, \beta}$. By Lemma 4.5 for all $k \in \mathbb{N}$ there exists $v_{k} \in N_{\tilde{\alpha}, \beta}$ such that $\mathcal{W}_{h}\left(v_{k}\right) \leq \mathcal{W}_{h}\left(u_{k}\right)$ and $v_{k}$ satisfies (4.6). Then Corollary 4.12 yields the claim.

\section{Properties of minimising sequences}

In the next two lemmas we achieve bounds on the derivative. We first observe that we can assume that when the graph of $u$ is above the line $y \mapsto \alpha y$ the derivative cannot be equal to $-\beta$. On the other hand, condition (4.6) (in particular (4.5)) gives bounds on the derivatives when the graph of $u$ is below the line $y \mapsto \alpha y$.

Lemma 4.14. Let $u \in N_{\alpha, \beta}$ satisfy (4.6). Then, there exists $v \in N_{\alpha, \beta}$ with lower Willmore energy than $u$ satisfying (4.6) and $v(x)<\alpha x$ for all $x \in(0,1)$ with $v^{\prime}(x)=-\beta$.

Proof. Let $x_{0}$ be the smallest element in $[0,1]$ such that $u^{\prime}\left(x_{0}\right)=-\beta$ and $u\left(x_{0}\right) \geq \alpha x_{0}$. If $x_{0}=1$ the claim follows with $v=u$. If $x_{0}<1$ and $u\left(x_{0}\right)=\alpha x_{0}$ the function $v(x)=u\left(x_{0} x\right) / x_{0}$, $x \in[-1,1]$, yields the claim. On the other hand, if $x_{0}<1$ and $u\left(x_{0}\right)>\alpha x_{0}$ by using a scaled version of Lemma 4.11 we "extend" the function $\left.u\right|_{\left[-x_{0}, x_{0}\right]}$ by inserting a cosh (see Figure 6). For each $\varrho \in\left(x_{0}, u\left(x_{0}\right) / \alpha_{\beta}\right)$ there exists $w_{\varrho} \in C^{1,1}([-\varrho, \varrho],(0, \infty))$ with lower Willmore energy than $\left.u\right|_{\left[-x_{0}, x_{0}\right]}$ such that $w_{\varrho}( \pm \varrho)=u\left(x_{0}\right)$ and $w_{\varrho}^{\prime}(\varrho)=-\beta$. We then choose $\varrho=u\left(x_{0}\right) / \alpha$ and $v$ to be equal to the function $w_{\varrho}$ rescaled to the interval $[-1,1]$. The choice of $\varrho$ is such that we extend $\left.u\right|_{\left[0, x_{0}\right]}$ until we touch the line $y \mapsto \alpha y$. Notice that $v \in N_{\alpha, \beta}$ and that $v$ satisfies (4.6). It remains to check that if $v^{\prime}(x)=-\beta$ for some $x \in(0,1)$ then $v(x)<\alpha x$. By construction, the function $w_{\varrho}$ is given by

$$
w_{\varrho}(x):= \begin{cases}u\left(x+x_{0}-\varrho\right) & \text { if } r-x_{0}+\varrho \leq x \leq \varrho, \\ \frac{1}{\lambda} \cosh (\lambda x) & \text { if } 0 \leq x<r-x_{0}+\varrho\end{cases}
$$




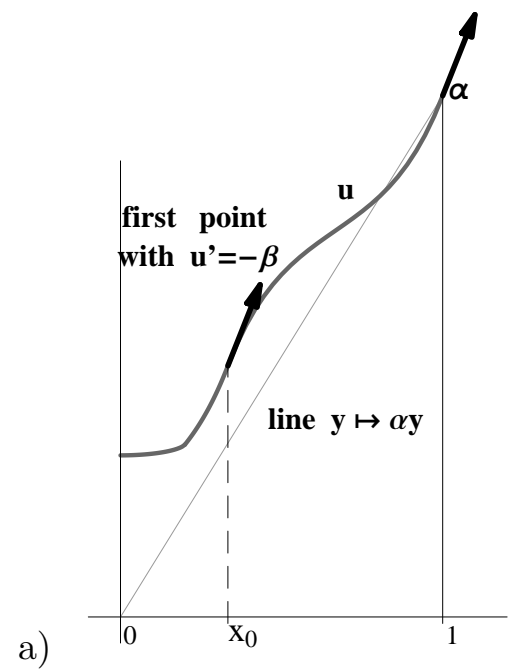

b)

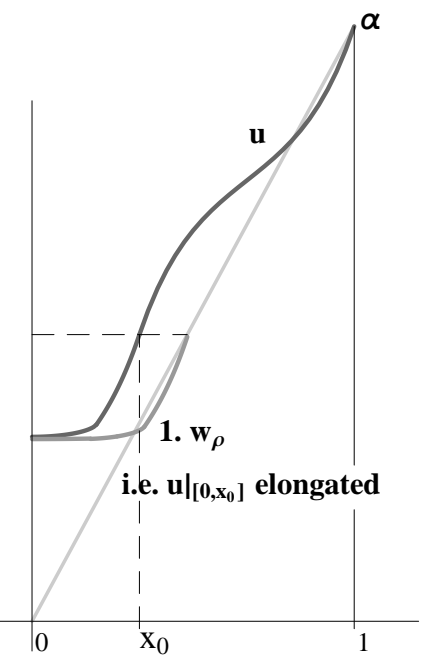

c)

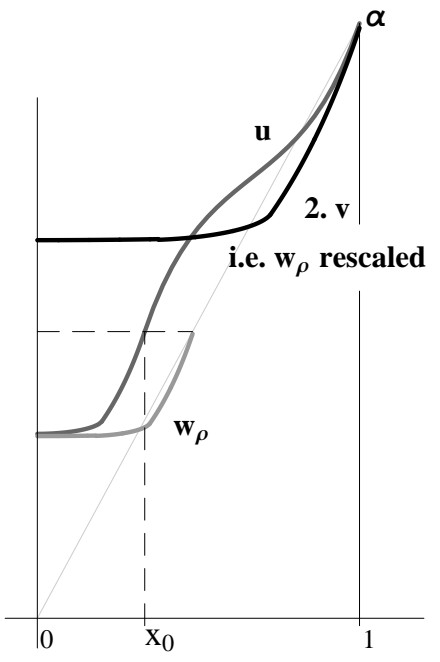

Figure 6: Proof of Lemma 4.14.

for some $r \in\left(0, x_{0}\right)$ and $\lambda=\lambda(r)>0$. If there exists $x \in(0,1)$ such that $v^{\prime}(x)=-\beta$ then $w_{\varrho}^{\prime}(\varrho x)=-\beta$. If $\varrho x \in\left[0, r-x_{0}+\varrho\right]$ then $w_{\varrho}^{\prime}(\varrho x)=-\beta$ implies $\lambda \varrho x=b$ and hence

$$
\frac{v(x)}{x}=\frac{w_{\varrho}(\varrho x)}{\varrho x}=\frac{\cosh (\lambda \varrho x)}{\lambda \varrho x}=\alpha_{\beta}<\alpha .
$$

If instead $\varrho x \in\left(r+\varrho-x_{0}, \varrho\right]$ then $u^{\prime}\left(\varrho x+x_{0}-\varrho\right)=-\beta$ and hence $u\left(\varrho x+x_{0}-\varrho\right)<\alpha\left(\varrho x+x_{0}-\varrho\right)$ from which it follows

$$
\frac{v(x)}{x}=\frac{u\left(\varrho x+x_{0}-\varrho\right)}{\varrho x}=\frac{u\left(\varrho x+x_{0}-\varrho\right)}{\varrho x+x_{0}-\varrho} \frac{\varrho x+x_{0}-\varrho}{\varrho x}<\alpha .
$$

The claim follows.

In the proof we use only that $\alpha>\alpha_{\beta}$.

We recall the definition (4.7) of the positive real numbers $b_{2}=b_{2}(\alpha)$ and $b_{1}=b_{1}(\alpha)$ such that $\cosh \left(b_{2}\right) / b_{2}=\alpha=\cosh \left(b_{1}\right) / b_{1}$ and $b_{2}>b^{*}>b_{1}$, with $b^{*}$ being the solution of $\cosh \left(b^{*}\right)=$ $b^{*} \sinh \left(b^{*}\right)$.

Lemma 4.15. Let $u \in N_{\alpha, \beta}$ satisfy (4.6) and $u^{\prime}(x) \neq-\beta$ for all $x \in(0,1)$ with $u(x) \geq \alpha x$. Then, $u^{\prime}(x) \leq \sinh \left(b_{2}\right)$ in $[0,1]$.

Proof. We assume first in addition that there exists a left neighbourhood of 1 such that there we have $u(x) \leq \alpha x$. Such a neighbourhood always exists if $-\beta>\alpha$. By 3) in Remark 4.10 we have that $u^{\prime}(x) \leq \sinh \left(b_{2}\right)$ for all $x$ such that $u(x) \leq \alpha x$. Hence, when the graph of $u$ is below the line $y \mapsto \alpha y$ we have a bound for the derivative. Let now $x \in(0,1)$ be such that $u(x)>\alpha x$. We show that $u^{\prime}(x)<-\beta$. Let $x_{0}$ be the smallest element in $(x, 1)$ such that $u\left(x_{0}\right)=\alpha x_{0}$. Then $u^{\prime}\left(x_{0}\right) \leq \alpha \leq-\beta$. If we assume that $u^{\prime}(x) \geq-\beta$ then, by continuity, there exists $y \in\left[x, x_{0}\right]$ such that $u^{\prime}(y)=-\beta$. A contradiction. Hence $u^{\prime}(x)<-\beta<\sinh \left(b_{2}\right)$.

It remains to consider the case where there is no left neighbourhood of 1 such that there we have $u(x) \leq \alpha x$. Then, necessarily $-\beta=\alpha$, and we have a sequence $x_{k} \nearrow 1$ with $u\left(x_{k}\right)>\alpha x_{k}$. Looking at the first point right from $x_{k}$, where $u$ reaches $y \mapsto \alpha y$ shows that $u(x)>\alpha x$ on $\left[0, x_{k}\right]$. Otherwise the mean value theorem would yield a point $\xi \in(0,1)$ with $u(\xi) \geq \alpha \xi$ and $u^{\prime}(\xi)=\alpha=-\beta$, a contradiction. Letting $k \rightarrow \infty$ yields $u(x)>\alpha x$ on $[0,1)$. By $u^{\prime}(0)=0$ we conclude that $0 \leq u^{\prime}(x)<-\beta<\sinh \left(b_{2}\right)$ on $[0,1)$ in this case. 
The following proposition characterises suitably modified minimising sequences.

Proposition 4.16. Let $\left(u_{k}\right)_{k \in \mathbb{N}}$ be a minimising sequence for $M_{\alpha, \beta}$ in $N_{\alpha, \beta}$ such that $\mathcal{W}_{h}\left(u_{k}\right) \leq$ $M_{\alpha, \beta}+1$ for all $k \in \mathbb{N}$. Let $b_{2}=b_{2}(\alpha)$ and $b_{1}=b_{1}(\alpha)$ be as defined in (4.7). Then, there exists a minimising sequence $\left(v_{k}\right)_{k \in \mathbb{N}} \subset N_{\alpha, \beta}$ such that for all $k \in \mathbb{N}$ the function $v_{k}$ satisfies (4.6), $\mathcal{W}_{h}\left(v_{k}\right) \leq \mathcal{W}_{h}\left(u_{k}\right)$

$$
\sinh \left(b_{2}\right) \geq v_{k}^{\prime}(x)>0 \text { for all } x \in(0,1] \text { and } C_{\alpha, \beta} \leq v_{k}(x) \leq \frac{1}{b_{1}} \cosh \left(b_{1} x\right) \text { in }[-1,1] \text {, }
$$

with a constant $C_{\alpha, \beta}>0$ depending on $M_{\alpha, \beta}$, $\sinh \left(b_{2}\right)$ and $-\beta$.

Proof. By Lemmas 4.5, 4.14 and 4.15 for each $u_{k}$ there exists $v_{k} \in N_{\alpha, \beta}$ with lower Willmore energy than $u_{k}$ such that $v_{k}$ satisfies $(4.6)$ and $\sinh \left(b_{2}\right) \geq v_{k}^{\prime}>0$ in $(0,1]$. According to Remark 4.8 we may also achieve that $v_{k}(x) \leq \frac{1}{b_{1}} \cosh \left(b_{1} x\right)$. The estimate from below for $v_{k}$ follows from Lemma 4.9 . The constant $C_{\alpha, \beta}$ denotes the term on the right hand side of Lemma 4.9 with $\gamma=\sinh \left(b_{2}\right)$ and $x_{0}=0$. Notice that by the assumption $\mathcal{W}_{h}\left(u_{k}\right) \leq M_{\alpha, \beta}+1$ the constant $C_{\alpha, \beta}$ depends only on $M_{\alpha, \beta}, \sinh \left(b_{2}\right)$ and $-\beta$.

\section{Proof of the existence theorem}

The real numbers $b_{2}=b_{2}(\alpha)$ and $b_{1}=b_{1}(\alpha)$ are defined in (4.7) and such that $\cosh \left(b_{2}\right) / b_{2}=\alpha=$ $\cosh \left(b_{1}\right) / b_{1}$ and $b_{2} \geq b^{*} \geq b_{1}$, with $b^{*}$ the solution of $\cosh \left(b^{*}\right)=b^{*} \sinh \left(b^{*}\right)$.

Theorem 4.17 (Existence and regularity). For $\beta<0$ and $\alpha$ such that $\alpha>\alpha_{\beta}$ and $-\beta \geq \alpha$ there exists a function $u \in C^{\infty}([-1,1],(0, \infty))$ such that the corresponding surface of revolution $\Gamma \subset \mathbb{R}^{3}$ solves the Dirichlet problem (1.4). This solution is positive and symmetric, and it has the following properties:

$$
\sinh \left(b_{2}\right) \geq u^{\prime}(x)>0 \text { in }(0,1] \quad \text { and } \quad \frac{1}{b_{1}} \cosh \left(b_{1} x\right) \geq u(x) \geq C_{\alpha, \beta} \text { in }[-1,1]
$$

with a constant $C_{\alpha, \beta}>0$ depending on $M_{\alpha, \beta}$, $\sinh \left(b_{2}\right)$ and $-\beta$.

Proof. Let $\left(u_{k}\right)_{k \in \mathbb{N}} \in N_{\alpha, \beta}$ be a minimising sequence for $M_{\alpha, \beta}$ such that $\mathcal{W}_{h}\left(u_{k}\right) \leq M_{\alpha, \beta}+1$ for all $k \in \mathbb{N}$. By Proposition 4.16 we may assume that each element $u_{k}$ of the minimising sequence satisfies (4.6) and (4.11). The rest of the proof is on the same line as that of Theorem 3.11. Notice that since $u_{k}$ satisfies (4.6) for all $k \in \mathbb{N}$ then also $u$ satisfies (4.6) and so $u^{\prime}>0$ in $(0,1]$.

We can improve Proposition 4.13 by showing that the energy is strictly increasing in $\alpha$.

Proposition 4.18. Let $M_{\alpha, \beta}$ be defined as in (2.7) and $\alpha>\alpha_{\beta}$. Then, for $-\beta \geq \tilde{\alpha}>\alpha$ we have $M_{\tilde{\alpha}, \beta}>M_{\alpha, \beta}$.

Proof. Let $u \in N_{\widetilde{\alpha}, \beta}$ be a solution of (1.4) with boundary values $\widetilde{\alpha}, \beta$ as constructed in Theorem 4.17. Then $\mathcal{W}_{h}(u)=M_{\widetilde{\alpha}, \beta}$ and $u$ satisfies (4.6). We first notice that $u$ satisfies (4.6) with a strict inequality. Indeed, if there exists $x_{0} \in(0,1)$ such that

$$
1-\frac{1}{\sqrt{1+u^{\prime}\left(x_{0}\right)^{2}}} \cosh \left(\frac{\sqrt{1+u^{\prime}\left(x_{0}\right)^{2}}}{u\left(x_{0}\right)} x_{0}\right)=0,
$$

reasoning as in Lemma 4.5 and using that $u$ is the minimiser in $N_{\widetilde{\alpha}, \beta}$, it follows that $\left.u\right|_{\left[-x_{0}, x_{0}\right]}$ is equal to a catenoid. Then, $u$ being a solution of $(2.5)$, it follows that $u$ is a catenoid in $[-1,1]$. This is not possible since $\alpha>\alpha_{\beta}$. Hence, applying the procedure of Corollary 4.12 to a minimiser $u \in N_{\widetilde{\alpha}, \beta}$ yields a $v \in N_{\alpha, \beta}$ with strictly lower energy $\mathcal{W}_{h}(u)>\mathcal{W}_{h}(v)$. Since $\mathcal{W}_{h}(v) \geq M_{\alpha, \beta}$ the claim follows. Notice that the same reasoning shows that also this last inequality is strict. 


\subsubsection{The case $-\beta<\alpha$}

In this case we are not able to obtain a bound from above for the derivative for minimising sequences in $N_{\alpha, \beta}$. Possibly, a loss of compactness could occur. To avoid this problem we restrict the set on which we minimise by adding a constraint. We require the derivative to be bounded by $\alpha$.

We consider

$$
\widetilde{N}_{\alpha, \beta}=\left\{u \in N_{\alpha, \beta}: u^{\prime}(x) \leq \alpha \text { for all } x \in[0,1]\right\},
$$

and

$$
\widetilde{M}_{\alpha, \beta}=\inf _{u \in \widetilde{N}_{\alpha, \beta}} \mathcal{W}_{h}(u)
$$

The assumption $-\beta<\alpha$ ensures that $\widetilde{N}_{\alpha, \beta}$ is not empty.

Also in this case, by Lemma 4.5 and the subsequent remark, it is sufficient to consider functions $u \in \widetilde{N}_{\alpha, \beta}$ satisfying (4.6).

\section{Monotonicity of the optimal energy}

Proceeding as in the previous section we find:

Lemma 4.19. Assume that $u \in \widetilde{N}_{\alpha, \beta}$ satisfies (4.6). Then for each $\varrho \in\left(1, \alpha / \alpha_{\beta}\right)$ there exists $u_{\varrho} \in C^{1,1}([-\varrho, \varrho],(0, \infty))$ positive and symmetric such that $u_{\varrho}( \pm \varrho)=\alpha, u_{\varrho}^{\prime}(\varrho)=-\beta$, (4.5) is satisfied in $[0, \varrho]$ and $\alpha \geq u_{\varrho}^{\prime}>0$ in $(0, \varrho]$ as well as

$$
\int_{-\varrho}^{\varrho} \kappa_{h}\left[u_{\varrho}\right]^{2} d s_{h}\left[u_{\varrho}\right] \leq \mathcal{W}_{h}(u) .
$$

Proof. The proof is exactly as in Lemma 4.11. By the explicit expression of $u_{\varrho}$ and the convexity of cosh, we see that $u^{\prime} \leq \alpha$ implies $u_{\varrho}^{\prime} \leq \alpha$.

Corollary 4.20. For each $u \in \widetilde{N}_{\alpha, \beta}$ such that $u$ satisfies (4.6) and for each $\gamma \in\left[\alpha_{\beta}, \alpha\right)$ there exists a positive symmetric $v \in C^{1,1}([-1,1],(0, \infty))$ such that $v$ satisfies $(4.6), v( \pm 1)=\gamma, v^{\prime}(1)=-\beta$, $\alpha \geq v^{\prime}(x)>0$ for $x \in(0,1]$ and $\mathcal{W}_{h}(v) \leq \mathcal{W}_{h}(u)$.

Proposition 4.21. Let $\widetilde{M}_{\alpha, \beta}$ be defined as in (4.12). Then for $\tilde{\alpha} \geq \alpha$ we have $\widetilde{M}_{\tilde{\alpha}, \beta} \geq \widetilde{M}_{\alpha, \beta}$.

\section{Properties of minimising sequences}

The following lemma is the analogue of Lemma 4.14 in the case $\alpha \leq-\beta$.

Lemma 4.22. Let $u \in \widetilde{N}_{\alpha, \beta}$ satisfy (4.6). Then there exists $v \in \widetilde{N}_{\alpha, \beta}$ with lower Willmore energy than $u$ satisfying (4.6) and $v(x)>\alpha x$ as well as $v^{\prime}(x)<-\beta$ in $(0,1)$.

Proof. We first notice that $u(x)>\alpha x$ in $(0,1)$. If $u^{\prime}(x)<-\beta$ in $(0,1)$ then the claim follows with $v=u$. Otherwise let $x_{0} \in(0,1)$ be the smallest element in $(0,1)$ such that $u^{\prime}\left(x_{0}\right)=-\beta$. We repeat then the construction in Lemma 4.14. By using a scaled version of Lemma 4.19 we elongate the function $\left.u\right|_{\left[-x_{0}, x_{0}\right]}$ by inserting a cosh. For each $\varrho \in\left(x_{0}, u\left(x_{0}\right) / \alpha_{\beta}\right)$ there exists $w_{\varrho} \in C^{1,1}([-\varrho, \varrho],(0, \infty))$ with lower Willmore energy than $\left.u\right|_{\left[-x_{0}, x_{0}\right]}$ such that $w_{\varrho}( \pm \varrho)=u\left(x_{0}\right)$ and $w_{\varrho}^{\prime}(\varrho)=-\beta$. We then choose $\varrho=u\left(x_{0}\right) / \alpha$ and $v$ to be equal to the function $w_{\varrho}$ rescaled to the interval $[-1,1]$. The choice of $\varrho$ is such that we extend $\left.u\right|_{\left[0, x_{0}\right]}$ until we touch the line $y \mapsto \alpha y$. Notice that $v \in N_{\alpha, \beta}, v$ satisfies (4.6) and that by convexity of cosh we have $v^{\prime}(x)<-\beta$ in $[0,1)$. In particular $v \in \widetilde{N}_{\alpha, \beta}$. Compared with Lemma 4.14, the proof in this case is simpler since we are always above the line $y \mapsto \alpha y$. In $\left[0, x_{0}\right)$ there are no points with derivative $\geq-\beta$. With this construction we do not add such points. 
The following proposition characterises suitably modified minimising sequences.

Proposition 4.23. Let $\left(u_{k}\right)_{k \in \mathbb{N}}$ be a minimising sequence for $\widetilde{M}_{\alpha, \beta}$ in $\widetilde{N}_{\alpha, \beta}$ such that $\mathcal{W}_{h}\left(u_{k}\right) \leq$ $\widetilde{M}_{\alpha, \beta}+1$ for all $k \in \mathbb{N}$. Let $b_{1}=b_{1}(\alpha)$ as defined in (4.7). Then, there exists a minimising sequence $\left(v_{k}\right)_{k \in \mathbb{N}} \subset \widetilde{N}_{\alpha, \beta}$ such that for all $k \in \mathbb{N}$, $v_{k}$ satisfies $(4.6), \mathcal{W}_{h}\left(v_{k}\right) \leq \mathcal{W}_{h}\left(u_{k}\right)$ and

$$
-\beta \geq v_{k}^{\prime}(x)>0 \text { for all } x \in(0,1] \quad \text { and } \quad 0<\alpha+\beta \leq v_{k}(x) \leq \frac{1}{b_{1}} \cosh \left(b_{1} x\right) \text { in }[-1,1] \text {. }
$$

Proof. By Lemmas 4.5 and 4.22 for each $u_{k}$ there exists $v_{k} \in \widetilde{N}_{\alpha, \beta}$ with lower Willmore energy than $u_{k}$ such that $v_{k}$ satisfies (4.6) and $-\beta \geq v_{k}^{\prime}>0$ in $(0,1]$. According to Remark 4.8 we may also achieve that $v_{k}(x) \leq \frac{1}{b_{1}} \cosh \left(b_{1} x\right)$. The estimate from below of $v_{k}$ follows directly.

\section{Proof of the existence theorem}

We recall that $b_{1}=b_{1}(\alpha)$ is defined in (4.7) and it is such that $\cosh \left(b_{1}\right) / b_{1}=\alpha$ and $b_{1} \leq b^{*}$, with $b^{*}$ solution of $\cosh \left(b^{*}\right)=b^{*} \sinh \left(b^{*}\right)$.

Theorem 4.24 (Existence and regularity). For $\beta<0$ and $\alpha$ such that $\alpha>\alpha_{\beta}$ and $\alpha>-\beta$ there exists a function $u \in C^{\infty}([-1,1],(0, \infty))$ such that the corresponding surface of revolution $\Gamma \subset \mathbb{R}^{3}$ solves the Dirichlet problem (1.4). This solution is positive and symmetric, and it has the following properties:

$$
-\beta \geq u^{\prime}(x)>0 \text { in }(0,1] \quad \text { and } \quad \frac{1}{b_{1}} \cosh \left(b_{1} x\right) \geq u(x) \geq \alpha+\beta \text { in }[-1,1] .
$$

Proof. As in the proof of Theorem 3.11 we find a minimiser $u \in \widetilde{N}_{\alpha, \beta}$ of $\mathcal{W}_{h}$. This means that $u$ minimises $\mathcal{W}_{h}$ in the class of all positive and symmetric $H^{2}(-1,1)$-functions $v$ satisfying $v( \pm 1)=\alpha$, $v^{\prime}(+1)=-\beta$, and having first derivative bounded pointwise by $\alpha$. Moreover, since the elements of the minimising sequence satisfy (4.6) and (4.13) then $u$ satisfies also (4.6) and hence (4.14). Since $u^{\prime}(x) \leq-\beta<\alpha$ for $x \in[0,1]$, then for $|t|$ sufficiently small $u+t \varphi \in \widetilde{N}_{\alpha, \beta}$ for $\varphi \in H^{2}(-1,1)$ with $\varphi( \pm 1)=0=\varphi^{\prime}( \pm 1)$. Therefore, $u$ is an interior point of $\widetilde{N}_{\alpha, \beta}$ in $H^{2}(-1,1)$ and $u$ weakly solves $(2.5)$.

The proof of smoothness of the solution is as in [DDG, Theorem 3.9, Step 2].

Proceeding as in the proof of Proposition 4.18 one can show that the energy also in this case is strictly increasing in $\alpha$.

Proposition 4.25. Let $-\beta<\alpha$ and $\alpha>\alpha_{\beta}$. Let $\widetilde{M}_{\alpha, \beta}$ be as defined in (4.12). Then $\widetilde{M}_{\widetilde{\alpha}, \beta}>\widetilde{M}_{\alpha, \beta}$ for all $\widetilde{\alpha}$ such that $\widetilde{\alpha}>\alpha$.

\subsection{The case $\alpha<\alpha_{\beta}$}

In this case the height prescribed at the boundary is smaller than the height of the catenoid centered at 0 and having derivative $-\beta$ at $x=1$. This case is not simply the dual of the case $\alpha>\alpha_{\beta}$. The function $b \mapsto \cosh (b) / b$ has a unique minimum at $b^{*}=1.1996786 \ldots$ and its minimal value is $\alpha^{*}=1.5088795 \ldots$ (see (1.5)). Hence, when considering $\alpha<\alpha_{\beta}$ we have to consider two different cases: when $\alpha \geq \alpha^{*}$ and when $\alpha<\alpha^{*}$. The first case is, in some sense, the dual to the case $\alpha>\alpha_{\beta}$. The constructions and the methods of proof are similar. On the other hand, the case $\alpha<\alpha^{*}$ is completely different. Here, only parts of the functions $u \in N_{\alpha, \beta}$ close to $x=1$ can be compared with catenoids. 
It is useful to restrict further the functions we consider. This restriction is technically important for the case $\alpha<\alpha^{*}$ but, for the sake of a uniform presentation, we use it in the entire section. We restrict our study to, what we call, admissible functions. These admissible functions are, however, dense in the space of all symmetric $H^{2}((-1,1),(0, \infty))$-functions and so, one can stick to them in minimising the Willmore functional without any loss of generality.

Definition 4.26 (Admissible functions). A function $u \in C^{1,1}([-a, a],(0, \infty)), a>0$, is called admissible if it is positive, symmetric and if there exist finitely many points $0=x_{0}<x_{1}<x_{2}<$ $\cdots<x_{m}=a$ such that $\left.u\right|_{\left[x_{j}, x_{j+1}\right]}, j=0, \ldots, m-1$, is a polynomial of degree at least two, or equal to $\cosh (\lambda(x-d)) / \lambda$ for some $\lambda \in(0, \infty), d \in \mathbb{R}$, or an arc of a circle with centre on the $x$-axis or an arc of a solution of (1.4) with $\beta=0$ as constructed in Theorem 3.18.

In what follows, we only perform constructions for admissible functions which yield again admissible functions. In most cases the starting point will be polynomials.

In this section we first show that it is sufficient to consider functions having at most one critical point in $(0,1)$ and satisfying a condition dual to (4.6). Moreover, we employ the catenoids as comparison functions. We then show that the energy is monotonically decreasing in $\alpha$. To proceed we need to distinguish the cases $\alpha \geq \alpha^{*}$ and $\alpha<\alpha^{*}$. We explain later the strategy of proof in the two cases.

\subsubsection{First observations}

The next two lemmas correspond to Lemma 4.5 for $\alpha>\alpha_{\beta}$. There we could restrict ourselves to functions satisfying $u^{\prime}>0$ in $(0,1)$. Here we show that we can restrict ourselves to functions having at most one critical point in $(0,1)$.

Lemma 4.27. Let $u \in N_{\alpha, \beta}$ be an admissible function in the sense of Definition 4.26. Then, there exists an admissible function $v \in N_{\alpha, \beta}$ with lower Willmore energy than $u$ and having at most one critical point in $(0,1)$, i.e. either $v^{\prime}>0$ in $(0,1]$ or there exists $x_{0} \in(0,1)$ such that $v^{\prime}\left(x_{0}\right)=0$, $v^{\prime}>0$ in $\left(x_{0}, 1\right]$ and $v^{\prime}<0$ in $\left(0, x_{0}\right)$.

Proof. If $u$ does not satisfy $u^{\prime}>0$ in $(0,1]$ there exists $x_{0} \in(0,1)$ such that $u^{\prime}\left(x_{0}\right)=0$ and $u^{\prime}(x)>0$ in $\left(x_{0}, 1\right]$. We then replace $\left.u\right|_{\left[-x_{0}, x_{0}\right]}$ with an appropriately rescaled solution of (1.4) with boundary data $u\left(x_{0}\right)$ and 0 as constructed in Theorem 3.18. This rescaled function has strictly negative derivative in $\left(0, x_{0}\right)$ by Lemma 3.20. The obtained function $v$ yields the claim.

In the next result we give a condition corresponding to (4.5) in this case. Here we use both catenoids and geodesic circles. The condition $x+u(x) u^{\prime}(x) \geq 0$ was a consequence of (4.5) for $\alpha>\alpha_{\beta}$ but this is not the case here.

Lemma 4.28. Let $u \in N_{\alpha, \beta}$ be an admissible function in the sense of Definition 4.26. Then, there exists an admissible function $v \in N_{\alpha, \beta}$ and $x_{0} \in[0,1)$ with $v^{\prime}<0$ in $\left(0, x_{0}\right), v^{\prime}\left(x_{0}\right)=0, v^{\prime}>0$ in $\left(x_{0}, 1\right]$ and $\mathcal{W}_{h}(v) \leq \mathcal{W}_{h}(u)$. Moreover, $v$ satisfies

$$
1-\frac{1}{\sqrt{1+v^{\prime}(x)^{2}}} \cosh \left(\frac{\sqrt{1+v^{\prime}(x)^{2}}}{v(x)} x\right) \leq 0 \text { in }\left[x_{0}, 1\right]
$$

and

$$
x+v(x) v^{\prime}(x) \geq 0 \text { in }[0,1] .
$$


Proof. Let $w \in N_{\alpha, \beta}$ be the function constructed in Lemma 4.27. One has $\mathcal{W}_{h}(w) \leq \mathcal{W}_{h}(u)$. We denote by $x_{1} \in[0,1)$ the point such that $w^{\prime}>0$ in $\left(x_{1}, 1\right], w^{\prime}\left(x_{1}\right)=0$ and $w^{\prime}<0$ in $\left(0, x_{1}\right)$.

This function $w$ satisfies (4.15) in $x=1$ and in $x_{1}$. Indeed, in $x=1$ we find

$$
1-\frac{1}{\sqrt{1+\beta^{2}}} \cosh \left(\frac{\sqrt{1+\beta^{2}}}{\alpha}\right)=\frac{1}{\alpha_{\beta}}\left(\alpha_{\beta}-\frac{1}{b} \cosh \left(\frac{\alpha_{\beta}}{\alpha} b\right)\right)<0
$$

since $\beta=-\sinh (b), \alpha_{\beta}=\frac{1}{b} \cosh (b)$, using (4.1) and $\alpha<\alpha_{\beta}$. In $x_{1}$ we get $1-\cosh \left(x_{1} / w\left(x_{1}\right)\right) \leq 0$ and equality holds only if $x_{1}=0$. If $w$ satisfies $(4.15)$ in $\left[x_{1}, 1\right]$ we define in $[0,1]$ the function $h(x):=x+w(x) w^{\prime}(x)$. The function $h$ is strictly positive in $\left[x_{1}, 1\right]$ since $w^{\prime} \geq 0$ in $\left[x_{1}, 1\right]$. Moreover, $h(0)=0$. If $h>0$ in $\left(0, x_{1}\right]$ the claim follows with $v=w$ and $x_{0}=x_{1}$. Otherwise there exists a biggest element $\bar{x} \in\left(0, x_{1}\right)$ with $h(\bar{x})=0$. Then we may substitute $w$ in $[-\bar{x}, \bar{x}]$ in a $C^{1,1}$-smooth way by an arc of a circle lowering the Willmore energy. This new function gives the claim.

It remains to treat the case when $w$ does not satisfy (4.15) in $\left[x_{1}, 1\right]$. For easy reference we denote here by $g$ the function on the left hand side of (4.15) with $v$ replaced by $w$. Let $x_{2}$ be the biggest element in $\left[x_{1}, 1\right]$ such that $g\left(x_{2}\right)=0$ and $g(x) \leq 0$ in $\left[x_{2}, 1\right]$. By Lemma 4.3 with $a=1$ and $f=w$ we can define a new function $v$ coinciding with $u$ on $\left[x_{2}, 1\right]$ and being a cosh on $\left[0, x_{2}\right]$. Since $v^{\prime}(0)=0$ we may extend it by symmetry to a $C^{1,1}$-function on $[-1,1]$. This new function always satisfies (4.15) and has lower Willmore energy. Notice that in this case $x_{0}=0$ and hence, (4.16) is certainly satisfied in $[0,1]$.

In what follows we consider only admissible functions $u \in N_{\alpha, \beta}$ satisfying the following conditions.

There exists $x_{0} \in[0,1)$ such that $u^{\prime}>0$ in $\left(x_{0}, 1\right], u^{\prime}\left(x_{0}\right)=0, u^{\prime}<0$ in $\left(0, x_{0}\right)$,

$$
\begin{gathered}
1-\frac{1}{\sqrt{1+u^{\prime}(x)^{2}}} \cosh \left(\frac{\sqrt{1+u^{\prime}(x)^{2}}}{u(x)} x\right) \leq 0 \text { in }\left[x_{0}, 1\right], \\
\text { and } x+u(x) u^{\prime}(x) \geq 0 \text { in }[0,1] .
\end{gathered}
$$

Before proceeding by proving monotonicity of the energy, we first compare functions in $N_{\alpha, \beta}$ with arcs of catenoids. In the next lemma we show that without loss of generality we may assume that functions satisfying (4.17) with $x_{0}>0$ satisfies also an uniform bound from below for $u\left(x_{0}\right) / x_{0}$.

Lemma 4.29. Let $u \in N_{\alpha, \beta}$ be an admissible function in the sense of Definition 4.26 satisfying (4.17) for some $x_{0}>0$. Then, there exists an admissible function $v \in N_{\alpha, \beta}$ such that $\mathcal{W}_{h}(u) \geq$ $\mathcal{W}_{h}(v)$, $v$ satisfies (4.17) for some $x_{1}>0$ and $v\left(x_{1}\right) \geq \frac{\alpha}{\operatorname{arsinh}(-\beta)\left(\alpha_{\beta}-\alpha\right)} x_{1}$.

Proof. We recall that $b=\operatorname{arsinh}(-\beta)$. If $u\left(x_{0}\right) \geq \frac{\alpha}{b\left(\alpha_{\beta}-\alpha\right)} x_{0}$ the claim follows with $v=u$. Otherwise we can construct a function satisfying the claim and with lower Willmore energy than $u$. We consider, starting from 1 and going towards 0 , the arc of the catenoid going through $(1, \alpha)$ and having derivative $-\beta$ in 1 . This is $x \mapsto \alpha \cosh \left(b \alpha_{\beta}\left(x-1+\alpha / \alpha_{\beta}\right) / \alpha\right) / b \alpha_{\beta}$. We follow the catenoid up to its minimum. Since $\alpha<\alpha_{\beta}$, the minimum is achieved in the point $1-\alpha / \alpha_{\beta} \in(0,1)$. In this point, we attach to this catenoid a suitably rescaled solution of (1.4) as constructed in Theorem 3.18 with boundary data $\alpha b / \alpha_{\beta}$ and 0 . Finally, extending the graph by symmetry, we obtain a function $v$. Notice that $v$ satisfies (4.17) with $x_{1}=1-\alpha / \alpha_{\beta}$ and that $v\left(x_{1}\right)=\frac{\alpha}{b\left(\alpha_{\beta}-\alpha\right)} x_{1}$. By the monotonicity property of the energy in the case $\beta=0$ (see Proposition 3.19) one sees that $v$ has lower Willmore energy than $u$. Notice that the cosh-part has the lowest possible energy among all curves connecting the boundary point $\alpha$ with slope $-\beta$ and any point with horizontal tangent. 
a)

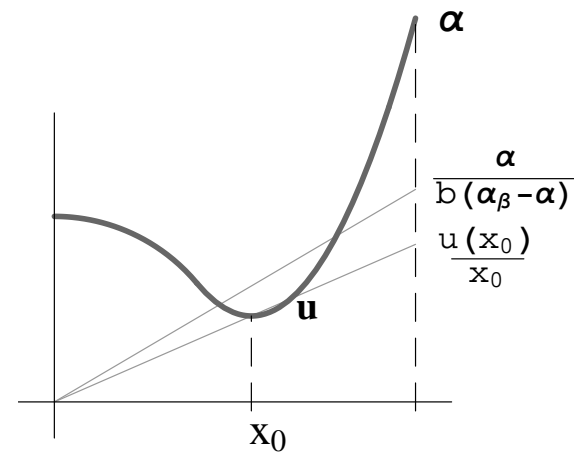

b)

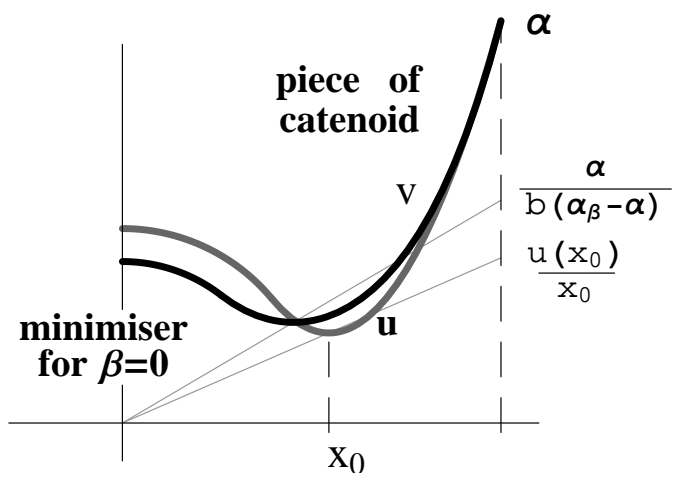

Figure 7: The construction in Lemma 4.29. On the left: a function $u \in N_{\alpha, \beta}$ with $u\left(x_{0}\right)<$ $\alpha x_{0} /\left(b\left(\alpha_{\beta}-\alpha\right)\right)$. On the right: a function $v \in N_{\alpha, \beta}$ with lower Willmore energy than $u$ satisfying $v\left(x_{1}\right)=\alpha x_{1} /\left(b\left(\alpha_{\beta}-\alpha\right)\right)$ with $x_{1}=1-\alpha / \alpha_{\beta}$.

The next lemma corresponds to Remark 4.10 in the case $\alpha>\alpha_{\beta}$. We recall here that for $\alpha^{\prime} \geq \alpha^{*}$, the real numbers $b_{2}=b_{2}\left(\alpha^{\prime}\right)$ and $b_{1}=b_{1}\left(\alpha^{\prime}\right)$ are defined in (4.7) by $\cosh \left(b_{2}\right) / b_{2}=\alpha^{\prime}=$ $\cosh \left(b_{1}\right) / b_{1}$ and $b_{2} \geq b^{*} \geq b_{1}$, where $b^{*}$ is the solution of $\cosh \left(b^{*}\right)=b^{*} \sinh \left(b^{*}\right)$.

Lemma 4.30. Let $u \in N_{\alpha, \beta}$ be an admissible function in the sense of Definition 4.26 and let $u$ satisfy (4.17) for some $x_{0} \in[0,1)$. Consider $\alpha^{\prime} \geq \alpha^{*}$. Then for all $x \in\left(x_{0}, 1\right]$ such that $u(x)>\alpha^{\prime} x$ we have that either $u^{\prime}(x)<\sinh \left(b_{1}\left(\alpha^{\prime}\right)\right)$ or $u^{\prime}(x)>\sinh \left(b_{2}\left(\alpha^{\prime}\right)\right)$.

Proof. The claim follows directly using that $u$ satisfies in particular (4.15).

The previous lemma shows that when the graph of $u \in N_{\alpha, \beta}$ is above the line $y \mapsto \alpha^{\prime} y, \alpha^{\prime}>\alpha^{*}$, we have a bound on the derivative. For this reason it is natural to distinguish below the cases $\alpha \geq \alpha^{*}$ and $\alpha<\alpha^{*}$.

\subsubsection{Monotonicity of the optimal energy}

We prove here that $M_{\alpha, \beta}$ decreases when $\alpha$ increases to $\alpha_{\beta}$. For later use we work in a more general setting.

We start by showing that we can construct functions defined in a smaller interval, with the same boundary values and with lower Willmore energy. We first prove the result for functions satisfying (4.17) with $x_{0}=0$ and then extend it to the general case.

Lemma 4.31. Fix $t<0$. Assume that $u \in C^{1,1}([-1,1],(0, \infty))$ is an admissible function in the sense of Definition 4.26 satisfying $u(1)<\alpha_{t}, u^{\prime}(1)=-t$ and (4.17) with $x_{0}=0$. Then for each $\varrho \in\left(u(1) / \alpha_{t}, 1\right)$ there exists an admissible function $u_{\varrho} \in C^{1,1}([-\varrho, \varrho],(0, \infty))$ such that $u_{\varrho}( \pm \varrho)=u(1), u_{\varrho}^{\prime}(\varrho)=-t, u_{\varrho}^{\prime}>0$ in $(0, \varrho], u_{\varrho}$ satisfies $(4.15)$ in $[0, \varrho]$ (with $\left.x_{0}=0\right)$ as well as

$$
\int_{-\varrho}^{\varrho} \kappa_{h}\left[u_{\varrho}\right]^{2} d s_{h}\left[u_{\varrho}\right] \leq \mathcal{W}_{h}(u) .
$$

Proof. One uses the same construction as in Lemma 4.11. Since $\alpha<\alpha_{\beta}$, this procedure now shortens the original function.

Lemma 4.32. Fix $t<0$. Assume that $u \in C^{1,1}([-1,1],(0, \infty))$ is an admissible function in the sense of Definition 4.26 satisfying $u^{\prime}(1)=-t, u(1)<\alpha_{t}$ and (4.17) for some $x_{0} \in[0,1)$. 
Then for each $\varrho \in\left(u(1) / \alpha_{t}, 1\right)$ there exists an admissible function $u_{\varrho} \in C^{1,1}([-\varrho, \varrho],(0, \infty))$ such that $u_{\varrho}( \pm \varrho)=u(1), u_{\varrho}^{\prime}(\varrho)=-t$. Moreover, there exists an $x_{1} \in[0, \varrho)$ with $u_{\varrho}^{\prime}\left(x_{1}\right)=0, u^{\prime}>0$ in $\left(x_{1}, \varrho\right]$ and $u_{\varrho}^{\prime}<0$ in $\left(0, x_{1}\right), u_{\varrho}$ satisfies $(4.15)$ in $\left[x_{1}, \varrho\right], x+u_{\varrho}(x) u_{\varrho}^{\prime}(x) \geq 0$ in $[0, \varrho]$ as well as

$$
\int_{-\varrho}^{\varrho} \kappa_{h}\left[u_{\varrho}\right]^{2} d s_{h}\left[u_{\varrho}\right] \leq \mathcal{W}_{h}(u) .
$$

Proof. It combines the constructions of Lemmas 3.3 and 3.16 (inserting circular arcs) and those of Lemma 4.31 (inserting catenoidal parts). See Figure 8. We emphasise that these constructions preserve the strict inequalities for the derivatives. The additional properties of $u_{\varrho}$ are ensured by possibly inserting once more a circular arc or a cosh, respectively, into the shortened function. Observe that (4.15) is certainly satisfied in $x=\varrho$ for any shortened function.

Notice that if $u\left(x_{0}\right) \geq \mu x_{0}$ then also $u_{\varrho}\left(x_{1}\right) \geq \mu x_{1}$.

a)

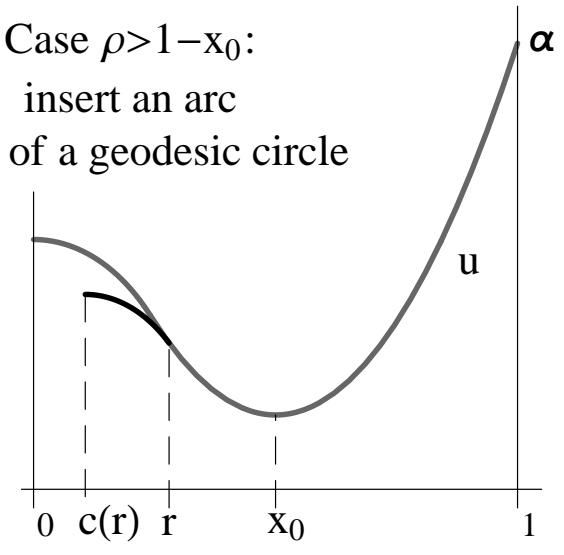

b)

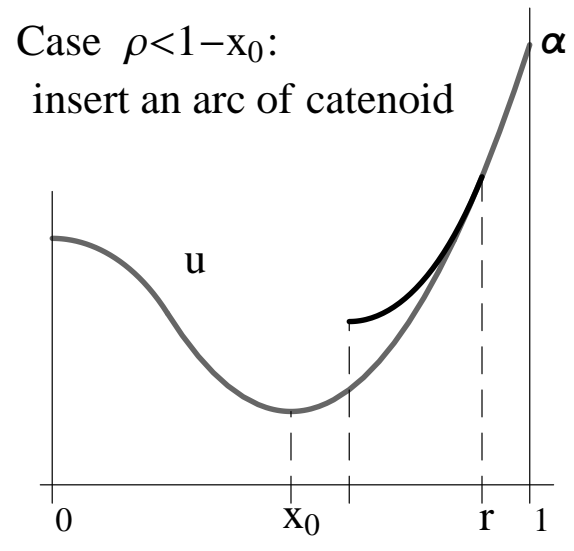

Figure 8: Proof of Lemma 4.32.

Corollary 4.33. Assume that $u \in N_{\alpha, \beta}$ satisfies (4.17) for some $x_{0} \in[0,1)$ and that $u$ is an admissible function in the sense of Definition 4.26. Then for all $\gamma \in\left(\alpha, \alpha_{\beta}\right]$ we find an admissible function $v \in C^{1,1}([-1,1],(0, \infty))$ satisfying (4.17) for some $x_{1} \in[0,1), v( \pm 1)=\gamma, v^{\prime}(1)=-\beta$ as well as $\mathcal{W}_{h}(v) \leq \mathcal{W}_{h}(u)$.

Proof. The claim follows from Lemma 4.32 by rescaling and taking $t=\beta$ and $u(1)=\alpha$.

Before showing monotonicity of the optimal Willmore energy, we prove a result being dual to Lemma 4.14.

Lemma 4.34. Let $u \in N_{\alpha, \beta}$ be an admissible function in the sense of Definition 4.26 satisfying (4.17) for some $x_{0} \in[0,1)$. Then, there exists an admissible function $v \in N_{\alpha, \beta}$ with lower Willmore energy than $u$ satisfying (4.17) for some $x_{1} \in[0,1)$ and $v(x)>\alpha x$ for all $x \in(0,1)$ with $v^{\prime}(x)=$ $-\beta$.

Proof. Let $\bar{x}$ be the smallest element in $[0,1]$ such that $u^{\prime}(\bar{x})=-\beta$ and $u(\bar{x}) \leq \alpha \bar{x}$. If $\bar{x}=1$ the claim follows with $v=u$ and $x_{1}=x_{0}$. If $\bar{x}<1$ and $u(\bar{x})=\alpha \bar{x}$ the function $v(x)=u(\bar{x} x) / \bar{x}$, $x \in[-1,1]$, yields the claim with $x_{1}=x_{0} / \bar{x}$. Finally, if $\bar{x}<1$ and $u(\bar{x})<\alpha \bar{x}$ by using a scaled version of Lemma 4.32 we shorten the function $\left.u\right|_{[-\bar{x}, \bar{x}]}$ by inserting a cosh or an arc of a circle. For each $\varrho \in\left(u(\bar{x}) / \alpha_{\beta}, \bar{x}\right)$ there exists $w_{\varrho} \in C^{1,1}([-\varrho, \varrho],(0, \infty))$ with lower Willmore energy than 
$\left.u\right|_{[-\bar{x}, \bar{x}]}$ such that $w_{\varrho}( \pm \varrho)=u(\bar{x})$ and $w_{\varrho}^{\prime}(\varrho)=-\beta$. We then choose $\varrho=u(\bar{x}) / \alpha$ such that the graph of $\left.u\right|_{[-\bar{x}, \bar{x}]}$ is shortened until we touch the line $y \mapsto \alpha y$. We define $v$ to be equal to the function $w_{\varrho}$ rescaled to the interval $[-1,1]$. Notice that $v \in N_{\alpha, \beta}$ is an admissible function and satisfies (4.17) for some $x_{1} \in[0,1)$. It remains to check that if $v^{\prime}(x)=-\beta$ for some $x \in(0,1)$ then $v(x)>\alpha x$. The function $w_{\varrho}$ is given by

$$
w_{\varrho}(x):= \begin{cases}u(x+\bar{x}-\varrho) & \text { if } r \leq x \leq \varrho, \\ g(x) & \text { if } 0 \leq x<r\end{cases}
$$

for some $r \in(0, \varrho)$, and either $g(x)=\cosh (\lambda x) / \lambda$ for some $\lambda>0$ or $g$ is an arc of a geodesic circle and $g^{\prime} \leq 0$. If there exists $x \in(0,1)$ such that $v^{\prime}(x)=-\beta$ then $w_{\varrho}^{\prime}(\varrho x)=-\beta$. Then, either $\varrho x \in[r, \varrho)$ and $w_{\varrho}(\varrho x)=u(\varrho x+\bar{x}-\varrho)$, or $\varrho x \in[0, r)$ and $w_{\varrho}(\varrho x)=\cosh (\lambda \varrho x) / \lambda$. In the first case, since $\varrho x+\bar{x}-\varrho<\bar{x}$ and $\bar{x}$ is the smallest element such that $u^{\prime}(\bar{x})=-\beta$ and $u(\bar{x}) \leq \alpha \bar{x}$, then $w_{\varrho}(\varrho x)=u(\varrho x+\bar{x}-\varrho)>\alpha(\varrho x+\bar{x}-\varrho)$ and so, by $\varrho<\bar{x}$

$$
\frac{v(x)}{x}=\frac{w_{\varrho}(\varrho x)}{\varrho x}=\frac{u(\varrho x+\bar{x}-\varrho)}{\varrho x}>\frac{\alpha(\varrho x+\bar{x}-\varrho)}{\varrho x}>\alpha .
$$

In the second case, if $w_{\varrho}^{\prime}(\varrho x)=-\beta$ then necessarily $\varrho \lambda x=b$ and so

$$
\frac{v(x)}{x}=\frac{w_{\varrho}(\varrho x)}{\varrho x}=\frac{\cosh (\lambda \varrho x)}{\lambda \varrho x}=\alpha_{\beta}>\alpha .
$$

The claim follows.

Note that $u\left(x_{0}\right) \geq \mu x_{0}$ implies that $v\left(x_{1}\right) \geq \mu x_{1}$.

a)

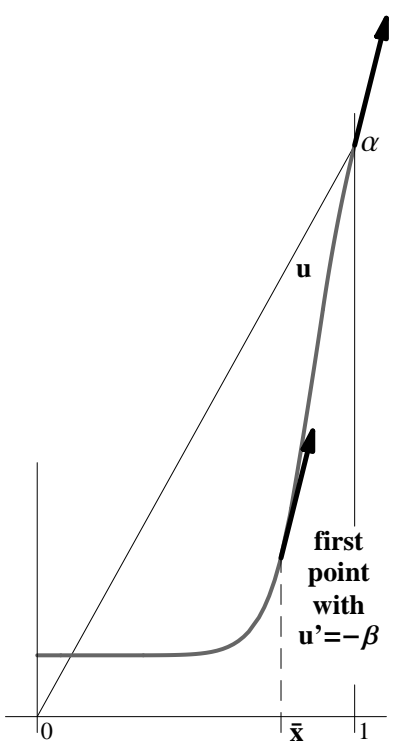

b)

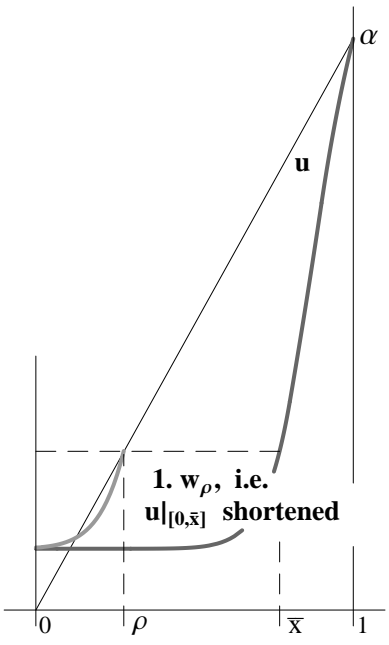

c)

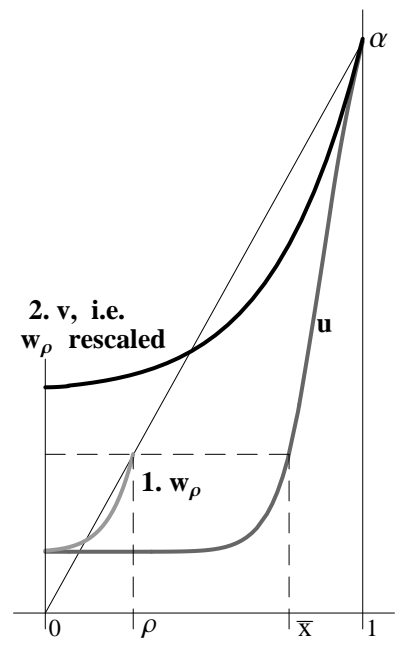

Figure 9: The three main steps of the proof of Lemma 4.34.

Proposition 4.35. Let $M_{\alpha, \beta}$ be defined as in (2.7) and $\alpha<\alpha_{\beta}$. Then, for $\tilde{\alpha} \leq \alpha$ we have $M_{\tilde{\alpha}, \beta} \geq M_{\alpha, \beta}$.

Proof. By density, we may choose a minimising sequence $\left(u_{k}\right)_{k \in \mathbb{N}} \subset N_{\alpha, \beta}$ for $M_{\tilde{\alpha}, \beta}$ consisting of positive symmetric polynomials of degree at least two. These functions are in particular admissible in the sense of Definition 4.26. Then, by Lemma 4.28 there exists a sequence $\left(v_{k}\right)_{k \in \mathbb{N}} \subset N_{\tilde{\alpha}, \beta}$ of admissible functions such that $v_{k}$ satisfies (4.17) for some $x_{k} \in[0,1)$ and $\mathcal{W}_{h}\left(v_{k}\right) \leq \mathcal{W}_{h}\left(u_{k}\right)$. Corollary 4.33 then yields the claim. 


\subsubsection{The case $\alpha \geq \alpha^{*}$}

In this case we can compare $u \in N_{\alpha, \beta}$ with the catenoids centered at 0 and going through $(1, \alpha)$. As observed in Remark 4.2, these are the functions $x \mapsto \cosh \left(b_{1} x\right) / b_{1}$ and $x \mapsto \cosh \left(b_{2} x\right) / b_{2}$ with $b_{1}=b_{1}(\alpha)$ and $b_{2}=b_{2}(\alpha)$ the positive real numbers such that $b_{1} \leq b^{*} \leq b_{2}$ and $\cosh \left(b_{1}\right) / b_{1}=$ $\alpha=\cosh \left(b_{2}\right) / b_{2}$, with $b^{*}$ the solution of $\cosh \left(b^{*}\right)=b^{*} \sinh \left(b^{*}\right)$. Since $\alpha<\alpha_{\beta}$ one sees that or $-\beta<\sinh \left(b_{1}\right)$ or $-\beta>\sinh \left(b_{2}\right)$ (see Figure 10). Notice that $\operatorname{since} \sinh \left(b_{1}\right) \leq \alpha \leq \sinh \left(b_{2}\right)$, these two cases correspond to the two cases $-\beta<\alpha$ and $-\beta \geq \alpha$ that we have treated separately also in the case $\alpha>\alpha_{\beta}$.
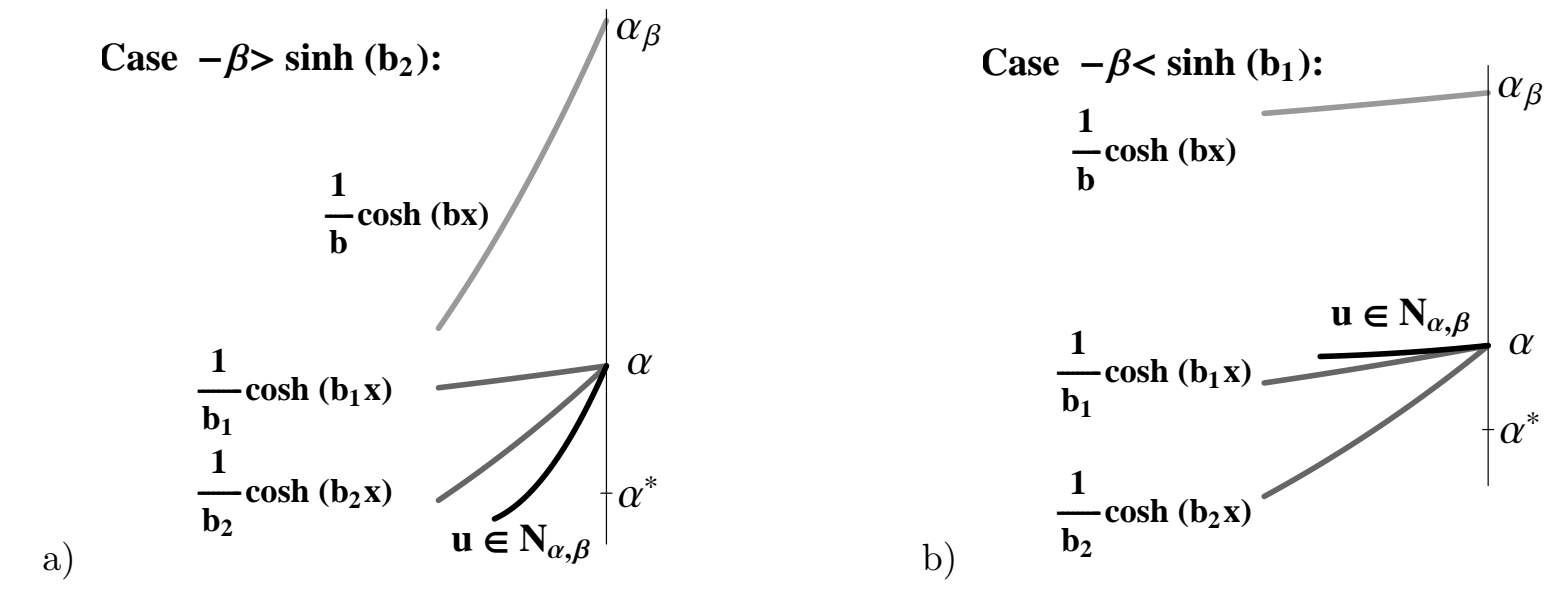

Figure 10: Two possibilities for the behaviour near 1 of the graph of $u \in N_{\alpha, \beta}$ when $\alpha^{*} \leq \alpha<\alpha_{\beta}$. Compare with Figure 4.

By comparing $u \in N_{\alpha, \beta}$ with the catenoids we show that in the case $-\beta<\sinh \left(b_{1}\right) \leq \alpha$ it is sufficient to consider functions $u \in N_{\alpha, \beta}$ such that $u(x) \geq \cosh \left(b_{1} x\right) / b_{1}$, i.e. remaining above the larger of the two catenoids. This in particular implies $u(x) \geq \alpha x$. This together with Lemma 4.30 gives bounds on the derivative. In the case $-\beta>\sinh \left(b_{2}\right) \geq \alpha$ we first prove bounds on the derivative using Lemmas 4.30 and 4.34. Then, by Lemma 4.9 we get a bound from below for the function.

\section{Properties of minimising sequences}

In the next lemmas it is convenient to distinguish the cases $-\beta<\sinh \left(b_{1}\right)$ and $-\beta>\sinh \left(b_{2}\right)$ because of the different behaviour with respect to the line $y \mapsto \alpha y$. Recall that $b_{1}=b_{1}(\alpha)$ and $b_{2}=b_{2}(\alpha)$ are the positive real numbers such that $b_{1} \leq b^{*} \leq b_{2}$ and $\cosh \left(b_{1}\right) / b_{1}=\alpha=\cosh \left(b_{2}\right) / b_{2}$, with $b^{*}$ being the solution of $\cosh \left(b^{*}\right)=b^{*} \sinh \left(b^{*}\right)$.

Lemma 4.36. We assume in addition that $-\beta<\sinh \left(b_{1}\right)$. Let $u \in N_{\alpha, \beta}$ be an admissible function in the sense of Definition 4.26. Assume furthermore that (4.17) is satisfied for some $x_{0} \in[0,1)$ and that $u^{\prime}(x) \neq-\beta$ for all $x \in[0,1)$ with $u(x) \leq \alpha x$. Then, $u(x) \geq \cosh \left(b_{1} x\right) / b_{1}$ and $u^{\prime}(x) \leq \sinh \left(b_{1} x\right)$ in $[0,1)$.

Proof. Since $-\beta<\sinh \left(b_{1}\right) \leq \alpha, u(x)>\cosh \left(b_{1} x\right) / b_{1}$ in a left neighbourhood of 1 . Moreover, since $u$ satisfies (4.17) (in particular (4.15) in $\left[x_{0}, 1\right]$ ) one sees as long as $u(x) \geq \cosh \left(b_{1} x\right) / b_{1}$ in $\left[x_{0}, 1\right]$ that

$$
\frac{\cosh \left(b_{1} x\right)}{b_{1} x} \leq \frac{u(x)}{x} \leq \frac{\cosh \left(\operatorname{arsinh}\left(u^{\prime}(x)\right)\right)}{\operatorname{arsinh}\left(u^{\prime}(x)\right)} .
$$

Since $\operatorname{arsinh}\left(u^{\prime}(1)\right)<b_{1} \leq b^{*}$, we conclude by continuity that $u^{\prime}(x) \leq \sinh \left(b_{1} x\right)$. Hence, $u(x)>$ $\frac{1}{b_{1}} \cosh \left(b_{1} x\right)$ on $\left[x_{0}, 1\right)$ and so, also in $[0,1)$. 
Lemma 4.37. We assume in addition that $-\beta>\sinh \left(b_{2}\right)$. Let $u \in N_{\alpha, \beta}$ be an admissible function in the sense of Definition 4.26. Assume furthermore that (4.17) is satisfied for some $x_{0} \in[0,1)$ and that $u^{\prime}(x) \neq-\beta$ for all $x$ with $u(x) \leq \alpha x$. Then, there exists an admissible function $v \in N_{\alpha, \beta}$ with lower Willmore energy than $u$, which satisfies (4.17) for some $x_{1} \in\left[0, x_{0}\right]$ and $v^{\prime}(x) \leq-\beta$ in $[0,1]$

Proof. Since $-\beta>\sinh \left(b_{2}\right) \geq \frac{\cosh \left(b_{2}\right)}{b_{2}}=\alpha, u(x)<\alpha x$ in a left neighbourhood of 1 . Let $x_{1}$ be the biggest element in $(0,1)$ such that $u\left(x_{1}\right)=\alpha x_{1}$ and $u^{\prime}\left(x_{1}\right) \leq \alpha$. Such an element exists since $u(0)>0$. Since $u^{\prime}\left(x_{1}\right) \leq \alpha<-\beta$ and $u^{\prime} \neq-\beta$ in $\left(x_{1}, 1\right)$ we have $u^{\prime} \leq-\beta$ in $\left[x_{1}, 1\right]$.

If $u^{\prime}\left(x_{1}\right)<\alpha$ then $u^{\prime}(x)<\alpha$ also in a left neighbourhood of $x_{1}$ and $u(x)>\alpha x$ in this neighbourhood. Hence by Lemma 4.30 for these points $u^{\prime}(x)<\sinh \left(b_{1}\right)$. Since $\sinh \left(b_{1}\right) \leq \frac{\cosh \left(b_{1}\right)}{b_{1}}=$ $\alpha$, then, by continuity, $u(x)>\alpha x$ and $u^{\prime}(x) \leq \sinh \left(b_{1}\right) \leq-\beta$ for all $x \in\left[0, x_{1}\right]$.

From Lemma 4.30 it follows also that $u^{\prime}\left(x_{1}\right)=\alpha$ can hold only if $\alpha=\alpha^{*} \operatorname{since} \sinh \left(b_{1}\right)<\alpha<$ $\sinh \left(b_{2}\right)$ for $\alpha \neq \alpha^{*}$. Hence for $\alpha>\alpha^{*}$ the claim is proved with $u=v$. If $\alpha=\alpha^{*}$ and $u^{\prime}\left(x_{1}\right)=\alpha^{*}$ then we substitute $u$ in $\left[-x_{1}, x_{1}\right]$ by the function $\cosh (\lambda x) / \lambda$ with $\lambda=b^{*} / x_{1}$ and $b^{*}$ defined in (1.5). We get a new function $v \in N_{\alpha, \beta}$ with lower Willmore energy than $u$ and such that $v$ satisfies (4.17) with $x_{1}=0, v^{\prime}(x) \leq \alpha^{*}$ and $v(x) \geq \alpha^{*} x$ in $\left[0, x_{1}\right]$

The following proposition characterises suitably modified minimising sequences.

Proposition 4.38. Let $\left(u_{k}\right)_{k \in \mathbb{N}}$ be a minimising sequence for $M_{\alpha, \beta}$ of admissible functions in the sense of Definition 4.26 in $N_{\alpha, \beta}$ such that $\mathcal{W}_{h}\left(u_{k}\right) \leq M_{\alpha, \beta}+1$ for all $k \in \mathbb{N}$. Then, there exists a minimising sequence $\left(v_{k}\right)_{k \in \mathbb{N}} \subset N_{\alpha, \beta}$ of admissible functions satisfying $(4.17), \mathcal{W}_{h}\left(v_{k}\right) \leq \mathcal{W}_{h}\left(u_{k}\right)$ and

$$
\max \left\{-\beta, \sinh \left(b_{1}\right)\right\} \geq v_{k}^{\prime}(x) \geq-\frac{\operatorname{arsinh}(-\beta)}{\alpha}\left(\alpha_{\beta}-\alpha\right) \text { and } C_{\alpha, \beta} \leq v_{k}(x) \leq \sqrt{1+\alpha^{2}-x^{2}}
$$

in $[0,1]$ with a constant $C_{\alpha, \beta}>0$ depending on $\alpha,-\beta$ and $M_{\alpha, \beta}$.

Proof. By Lemmas 4.28, 4.34, 4.36 if $-\beta<\sinh \left(b_{1}\right)$ or 4.37 if $-\beta>\sinh \left(b_{2}\right)$, and Lemma 4.29 for each $u_{k}$ there exists $v_{k} \in N_{\alpha, \beta}$ with lower Willmore energy than $u_{k}$ such that $v_{k}$ satisfies (4.17) for some $x_{k} \in[0,1)$ and

$$
v_{k}^{\prime}(x) \leq \max \left\{-\beta, \sinh \left(b_{1}\right)\right\} \quad \text { and } \quad v_{k}\left(x_{k}\right) \geq \frac{\alpha}{\operatorname{arsinh}(-\beta)\left(\alpha_{\beta}-\alpha\right)} x_{k} .
$$

Since $v_{k}$ satisfies $(4.16)$ we get $v_{k}(x) \leq \sqrt{1+\alpha^{2}-x^{2}}$ in $[0,1]$ and

$$
v_{k}^{\prime}(x) \geq-\frac{x}{v_{k}(x)} \geq-\frac{x_{k}}{v_{k}\left(x_{k}\right)} \geq-\operatorname{arsinh}(-\beta) \frac{\alpha_{\beta}-\alpha}{\alpha} \quad \text { for } x \in\left[0, x_{k}\right]
$$

while $v_{k}^{\prime} \geq 0$ in $\left[x_{k}, 1\right]$. The estimate from below for $v_{k}$ follows from the second estimate in (4.19) if $x_{k} \geq 1 / 2$ and from Lemma 4.9 if $x_{k} \leq 1 / 2$.

\section{Proof of the existence theorem}

We recall here that for $\alpha \geq \alpha^{*}, b_{1}=b_{1}(\alpha)$ denotes the positive real number such that $\cosh \left(b_{1}\right) / b_{1}=$ $\alpha$ and $b_{1} \leq b^{*}$ with $b^{*}$ being the solution of $\cosh \left(b^{*}\right)=b^{*} \sinh \left(b^{*}\right)$.

Theorem 4.39 (Existence and regularity). For $\beta<0$ and $\alpha$ such that $\alpha^{*} \leq \alpha<\alpha_{\beta}$ there exists a function $u \in C^{\infty}([-1,1],(0, \infty))$ such that the corresponding surface of revolution $\Gamma \subset \mathbb{R}^{3}$ solves 
the Dirichlet problem (1.4). This solution is positive and symmetric, has at most one critical point in $(0,1)$, and satisfies

$$
\begin{gathered}
\max \left\{\sinh \left(b_{1}\right),-\beta\right\} \geq u^{\prime}(x) \geq-\left(\alpha-\alpha_{\beta}\right) \frac{\operatorname{arsinh}(-\beta)}{\alpha} \text { in }(0,1] \\
\text { and } \sqrt{1+\alpha^{2}-x^{2}} \geq u(x) \geq C_{\alpha, \beta} \text { in }[-1,1],
\end{gathered}
$$

with a constant $C_{\alpha, \beta}>0$ depending on $M_{\alpha, \beta}, \alpha$ and $-\beta$.

Proof. By density of polynomials in $H^{2}(-1,1)$ a minimising sequence $\left(u_{k}\right)_{k \in \mathbb{N}}$ for $M_{\alpha, \beta}$ may be chosen in $N_{\alpha, \beta}$ which consists of positive symmetric polynomials of degree at least two and such that $\mathcal{W}_{h}\left(u_{k}\right) \leq M_{\alpha, \beta}+1$ for all $k \in \mathbb{N}$. By Proposition 4.38 we may assume that each element $u_{k}$ of the minimising sequence satisfies (4.18). The rest of the proof is along the lines of Theorem 3.11. Moreover, with the construction of Lemma 4.27 one can prove that $u$ has at most one critical point in $(0,1)$.

Reasoning as in the proof of Propositions 3.12 and 4.18, one can prove that the energy is strictly decreasing in $\alpha$.

Proposition 4.40. Let $M_{\alpha, \beta}$ be as defined in (2.7) and $\alpha^{*} \leq \alpha<\alpha_{\beta}$. Then $M_{\widetilde{\alpha}, \beta}>M_{\alpha, \beta}$ for all $\widetilde{\alpha} \in\left(\alpha^{*}, \alpha\right)$.

\subsubsection{The case $\alpha<\alpha^{*}$}

In this case no catenoid is going through the points $( \pm 1, \alpha)$ which $u \in N_{\alpha, \beta}$ can be compared with. However, the results from the previous subsection will be useful also here. Since $u \in N_{\alpha, \beta}$ is strictly positive, going from the right to the left, there exists certainly a first point $\bar{x}$ where $u(\bar{x})=\alpha^{*} \bar{x}$. From here on, we may refer to the geometric constructions which led to suitable minimising sequences as described in Proposition 4.38.

The difficulty is now to understand how the graph of a suitable function $u \in N_{\alpha, \beta}$ should behave or should be suitably modified before reaching the line $y \mapsto \alpha^{*} y$. By Lemma 4.34 it is sufficient to consider functions where $u^{\prime} \neq-\beta$ when we are below the line $y \mapsto \alpha y$. This result gives a bound on the derivative when the graph of $u$ is below the line $y \mapsto \alpha y$. Hence, it remains to get an estimate on $u^{\prime}$ on the set $\left\{x: \alpha x<u(x)<\alpha^{*} x\right\}$. To this end we study the function $u(x) / x$. In order to ensure that $u(x) / x$ has only finitely many oscillations in $[0,1]$ we restrict ourselves to admissible functions as defined in Definition 4.26. Going from the left to the right, we prove that the function $u(x) / x$ is decreasing from the first and only point where the graph of $u$ crosses the line $y \mapsto \alpha^{*} y$ to the point where it crosses or touches the line $y \mapsto \alpha y$. This leads to bounds for the derivative on suitably modified minimising sequences.

\section{Properties of minimising sequences}

We start by showing that, going from the right to the left, once the graph of $u$ reaches the line $y \mapsto \alpha^{*} y$, then one may achieve that it remains above this line.

Lemma 4.41. Let $u \in N_{\alpha, \beta}$ be an admissible function in the sense of Definition 4.26 satisfying (4.17) for some $x_{0} \in[0,1)$. Let $\bar{x}$ be such that $u(\bar{x})=\alpha^{*} \bar{x}$ and $u(x)<\alpha^{*} x$ in $(\bar{x}, 1]$.

Then there exists an admissible function $v \in N_{\alpha, \beta}$ with $\mathcal{W}_{h}(u) \geq \mathcal{W}_{h}(v)$ and satisfying (4.17) for some $x_{1} \in[0,1)$. Moreover, $v(\bar{x})=\alpha^{*} \bar{x}, v(x)<\alpha^{*} x$ in $(\bar{x}, 1]$ and $v(x) \geq \cosh \left(b^{*} x / \bar{x}\right) \bar{x} / b^{*}>\alpha^{*} x$ as well as $v^{\prime}(x)<\alpha^{*}$ in $[0, \bar{x})$. 
Proof. We have $u^{\prime}(\bar{x}) \leq \alpha^{*}$. If $u^{\prime}(\bar{x})<\alpha^{*}$ then $u(x)>\cosh \left(b^{*} x / \bar{x}\right) \bar{x} / b^{*}$ in a left neighbourhood of $\bar{x}$. Since $u$ satisfies (4.17) one sees as in the proof of Lemma 4.36 that $u(x)>\cosh \left(b^{*} x / \bar{x}\right) \bar{x} / b^{*}>$ $\alpha^{*} x$ and $u^{\prime}(x)<\alpha^{*}$ for all $x \in[0, \bar{x}]$. The claim then follows with $v=u$.

If instead $u^{\prime}(\bar{x})=\alpha^{*}$ then we substitute $u$ in $[-\bar{x}, \bar{x}]$ by $\cosh \left(b^{*} x / \bar{x}\right) \bar{x} / b^{*}$. We get a new admissible function $v \in N_{\alpha, \beta}$ with lower Willmore energy than $u$ and $v(x)>\alpha^{*} x$ as well as $v^{\prime}(x)<\alpha^{*}$ in $(0, \bar{x})$.

Notice that in the previous lemma if $u(x) \geq \alpha x$ then also $v(x) \geq \alpha x$. Moreover, if $u\left(x_{0}\right) \geq \mu x_{0}$ then also $v\left(x_{1}\right) \geq \mu x_{1}$.

a)

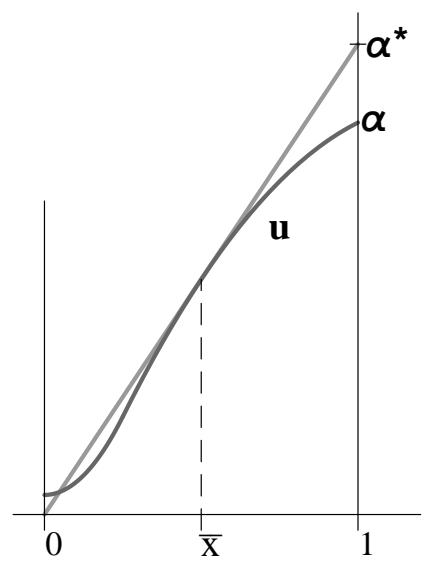

b)

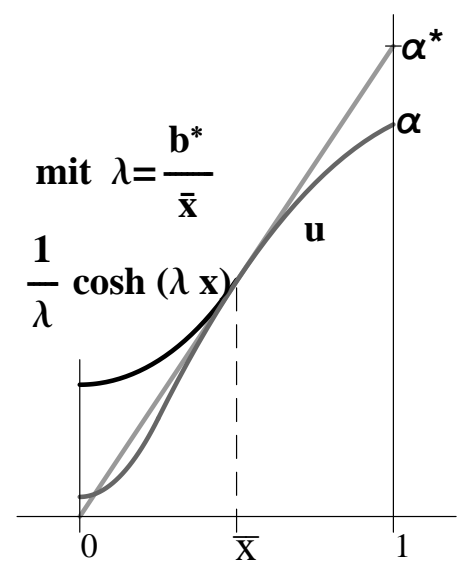

Figure 11: Proof of Lemma 4.41 in the case where $u \in N_{\alpha, \beta}$ is tangent in $\bar{x}$ to the line $y \mapsto \alpha^{*} y$.

The aim of the next constructions is to show for functions $u$ as in Lemma 4.41 one may achieve that $u(x) / x$ is decreasing for $x \in(0,1)$ where $\alpha x<u(x)<\alpha^{*} x$. As in Lemma 4.32, they are based on inserting parts of geodesic circles and catenoids to shorten the intervals and decrease the Willmore energy. Assuming $u(1)<\alpha^{*}$ we study now additional properties inherited by the shortened function $u_{\varrho}$.

In the next result we prove that if $u(x) / x$ is decreasing in $\left\{x \in(0,1): u(1) x \leq u(x) \leq \alpha^{*} x\right\}$ and $u(x) \geq u(1) x$ then, when $\varrho \geq u(1) / \alpha^{*}$, also $u_{\varrho}(x) / x$ is decreasing in $\{x \in(0, \varrho): u(1) x \leq$ $\left.u_{\varrho}(x) \leq \alpha^{*} x\right\}$ and $u_{\varrho}(x) \geq u(1) x / \varrho$. Notice that the result holds when we shorten the graph of $u$ until we reach the line $y \mapsto \alpha^{*} y$ but not until $y \mapsto \alpha_{\beta} y$.

Proposition 4.42. Fix $t<0$. Let $u \in C^{1,1}([-1,1],(0, \infty))$ be an admissible function in the sense of Definition 4.26 satisfying (4.17) for some $x_{0} \in[0,1), u(x) \geq u(1) x$ in $[0,1], u^{\prime}(1)=-t$ and $u(1)<\alpha^{*}$. Moreover, for $\varrho \in\left[u(1) / \alpha^{*}, 1\right]$, let $u_{\varrho}$ be the function constructed in Lemma 4.32. Then

$$
u_{\varrho}(x) \geq \frac{u(1)}{\varrho} x \text { for all } x \in[0, \varrho]
$$

and $u_{\varrho}$ satisfies $(4.17)$ on $[0, \varrho)$ for some $\hat{x} \in[0, \varrho)$.

Furthermore, if there exists $\bar{x} \in(0,1)$ such that $u(x) \geq \alpha^{*} x$ for all $x \in[0, \bar{x}], u(x)<\alpha^{*} x$ for all $x \in(\bar{x}, 1]$ and $u(x) / x$ is decreasing in $[\bar{x}, 1]$, then there exists $\widetilde{x} \in(0, \varrho)$ such that $u_{\varrho}(x) \geq \alpha^{*} x$ for all $x \in[0, \widetilde{x}], u_{\varrho}(x)<\alpha^{*} x$ for all $x \in(\widetilde{x}, \varrho]$ and $u_{\varrho}(x) / x$ is decreasing in $[\widetilde{x}, \varrho]$.

Proof. For $\varrho \in\left[u(1) / \alpha^{*}, 1\right]$ there exists $r^{\prime} \in[0, \varrho)$ such that $u_{\varrho}$ is given in $[0, \varrho]$ by

$$
u_{\varrho}(x)= \begin{cases}u(1+x-\varrho) & \text { if } r^{\prime} \leq x \leq \varrho, \\ f(x) & \text { if } 0 \leq x<r^{\prime}\end{cases}
$$


where $f$ is either an arc of a circle (and $u_{\varrho}^{\prime}\left(r^{\prime}\right) \leq 0$ ) or a $\cosh (\lambda x) / \lambda$ for $\lambda \in \mathbb{R}^{+}$. The first claim is satisfied in $\left[0, r^{\prime}\right]$ since if $f$ is a cosh then $u_{\varrho}(x) \geq \alpha^{*} x$ and $\alpha^{*} x \geq u(1) x / \varrho$ by assumption. On the other hand if $f$ is a circular arc then

$$
\frac{u_{\varrho}(x)}{x}=\frac{f(x)}{x} \geq \frac{f\left(r^{\prime}\right)}{r^{\prime}}=\frac{u\left(r^{\prime}+1-\varrho\right)}{r^{\prime}} \geq u(1) \frac{r^{\prime}+1-\varrho}{r^{\prime}} \geq \frac{u(1)}{\varrho} .
$$

For $x \in\left[r^{\prime}, \varrho\right]$ we have

$$
\frac{u_{\varrho}(x)}{x}=\frac{u(x+1-\varrho)}{x} \geq u(1) \frac{x+1-\varrho}{x} \geq \frac{u(1)}{\varrho} .
$$

For the second claim let $\widetilde{x}$ be the biggest element in $[0, \varrho]$ such that $u_{\varrho}(x) \geq \alpha^{*} x$ in $[0, \widetilde{x}]$. We first treat the case when $f(x)=\cosh (\lambda x) / \lambda$. Since $\cosh (\lambda x) / \lambda \geq \alpha^{*} x$ then $\widetilde{x} \geq r^{\prime}$. Moreover, since $u(\widetilde{x}+1-\varrho)=u_{\varrho}(\widetilde{x})=\alpha^{*} \widetilde{x}<\alpha^{*}(\widetilde{x}+1-\varrho)$ we necessarily have $\widetilde{x}+1-\varrho>\bar{x}$. So, for $x>\widetilde{x}$ we have

$$
u_{\varrho}(x)=\frac{u(x+1-\varrho)}{x+1-\varrho}(x+1-\varrho) \leq \frac{u(\widetilde{x}+1-\varrho)}{\widetilde{x}+1-\varrho}(x+1-\varrho)=\frac{\alpha^{*} \widetilde{x}}{\widetilde{x}+1-\varrho}(x+1-\varrho)<\alpha^{*} x,
$$

giving $u_{\varrho}(x)<\alpha^{*} x$ in $(\widetilde{x}, \varrho]$. Since $u(x) / x$ is decreasing in $(\bar{x}, 1]$ then $u^{\prime}(x) \leq u(x) / x$ in $(\bar{x}, 1]$. Using that $\bar{x}<\widetilde{x}+1-\varrho$ we find for $x \in(\widetilde{x}, \varrho]$

$$
u_{\varrho}^{\prime}(x)=u^{\prime}(x+1-\varrho) \leq \frac{u(x+1-\varrho)}{x+1-\varrho}=\frac{u_{\varrho}(x)}{x} \frac{x}{x+1-\varrho}<\frac{u_{\varrho}(x)}{x},
$$

showing that $u_{\varrho}(x) / x$ is decreasing in $[\widetilde{x}, \varrho]$.

If instead $f(x)$ is a circular arc of a circle we need to distinguish two cases. If $\widetilde{x} \geq r^{\prime}$ then we reason as in (4.20) and (4.21). If instead $\widetilde{x}<r^{\prime}$ then $u_{\varrho}(x)<\alpha^{*} x$ and $u_{\varrho}(x) / x$ is decreasing in $\left(\widetilde{x}, r^{\prime}\right]$ since $u_{\varrho}^{\prime} \leq 0$ in $\left[0, r^{\prime}\right]$. In particular $u\left(r^{\prime}+1-\varrho\right)=u_{\varrho}\left(r^{\prime}\right)<\alpha^{*} r^{\prime}$ and so $r^{\prime}+1-\varrho>\bar{x}$. It then follows for $x \in\left[r^{\prime}, \varrho\right]$ that

$$
u_{\varrho}(x)=\frac{u(x+1-\varrho)}{x+1-\varrho}(x+1-\varrho) \leq \frac{u\left(r^{\prime}+1-\varrho\right)}{r^{\prime}+1-\varrho}(x+1-\varrho)<\alpha^{*} \frac{r^{\prime}(x+1-\varrho)}{r^{\prime}+1-\varrho} \leq \alpha^{*} x,
$$

and proceeding as in (4.21) one shows that $u_{\varrho}(x) / x$ is decreasing also in $\left[r^{\prime}, \varrho\right]$.

Thanks to the previous proposition we may now show that if $u(x) \geq u(1) x$ in $[0,1]$ and $u(1)<\alpha^{*}$, then we can also assume that $u(x) / x$ is decreasing on the set $\left\{x \in(0,1]: u(x) \leq \alpha^{*} x\right\}$.

Proposition 4.43. Let $u \in C^{1,1}([-1,1],(0, \infty))$ be a admissible function in the sense of Definition 4.26 satisfying (4.17) for some $x_{0} \in[0,1), u^{\prime}(1)>0$ and $u(1)<\alpha^{*}$. We assume further that $u(x)>u(1) x$ in $(0,1)$ and that there exists $\bar{x} \in(0,1)$ with $u(x) \geq \alpha^{*} x$ in $[0, \bar{x}]$ and $u(x)<\alpha^{*} x$ in $(\bar{x}, 1]$.

Then, there exists a admissible function $v \in C^{1,1}([-1,1],(0, \infty))$ with $v^{\prime}(1)=u^{\prime}(1), u(1)=$ $v(1), \mathcal{W}_{h}(u) \geq \mathcal{W}_{h}(v)$ and satisfying (4.17) for some $\hat{x} \in[0,1)$. Moreover, there exists $\widetilde{x} \in(0,1)$ so that $v(x) \geq \alpha^{*} x$ in $[0, \widetilde{x}]$ and $v(x)<\alpha^{*} x$ for all $x \in(\widetilde{x}, 1]$ and $v(x) / x$ is decreasing in $[\widetilde{x}, 1]$.

Proof. By assumption $u(x) / x<u(\bar{x}) / \bar{x}$ in a right neighbourhood of $\bar{x}$ and $u(x) / x>u(1) / 1$ in a left neighbourhood of 1 . If $u(x) / x$ is decreasing in $[\bar{x}, 1]$ the claim follows with $v=u$. Otherwise there exists a first local minimum $x_{1}$ of $u(x) / x$ in $[\bar{x}, 1]$. By our definition of admissibility this minimum is strict. For easy notation let $\alpha^{\prime}$ denote $u\left(x_{1}\right) / x_{1}$. Notice that $u(1)<\alpha^{\prime}<\alpha^{*}$ and that $u^{\prime}\left(x_{1}\right)=\alpha^{\prime}$. Let $x_{3}$ be the smallest element in $\left(x_{1}, 1\right]$ with $u\left(x_{3}\right)=\alpha^{\prime} x_{3}$ and $x_{2}$ be the largest 
a)

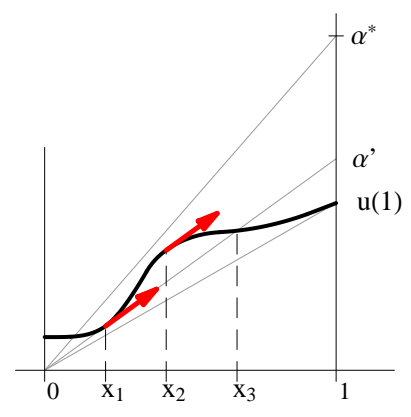

b)

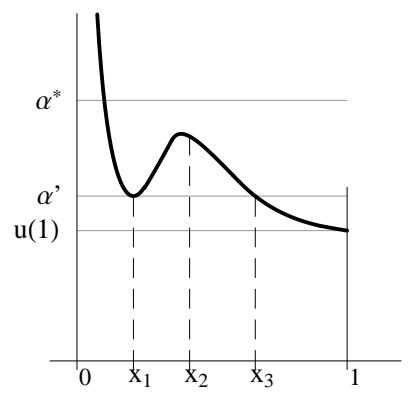

Figure 12: Proof of Proposition 4.43. In $x_{1}$ and $x_{2}$ the tangents are parallel. $\left.u\right|_{\left[-x_{1}, x_{1}\right]}$ is shortened and rescaled. So, we avoid oscillations of $x \mapsto u(x) / x$ decreasing the Willmore energy.

element in $\left(x_{1}, x_{3}\right)$ with $u^{\prime}\left(x_{2}\right)=\alpha^{\prime}$. Then $u(x)>\alpha^{\prime} x$ for $x \in\left(x_{1}, x_{3}\right)$. For $x \in\left(x_{2}, x_{3}\right)$ we see that $x^{2}\left(\frac{u}{x}\right)^{\prime}=x u^{\prime}-u<x \alpha^{\prime}-x \alpha^{\prime}=0$, i.e. $x \mapsto \frac{u}{x}$ is strictly decreasing on $\left(x_{2}, x_{3}\right)$ so that it has a local maximum on $\left(x_{1}, x_{2}\right)$.

The idea is to replace $\left.u\right|_{\left[-x_{2}, x_{2}\right]}$ by the appropriately shortened and rescaled $\left.u\right|_{\left[-x_{1}, x_{1}\right]}$ according to Proposition 4.42. For this new function $v$ the number of local extrema of $x \mapsto v(x) / x$ below the line $y \mapsto \alpha^{*} y$ has decreased by at least two. Since $x \mapsto u(x) / x$ has only finitely many local extrema, after finitely many iterations we obtain the claim. We present this argument now in detail.

By using a scaled version of Proposition 4.42 we shorten the function $\left.u\right|_{\left[-x_{1}, x_{1}\right]}$ by inserting a cosh or an arc of a circle. For each $\varrho \in\left[u\left(x_{1}\right) / \alpha^{*}, x_{1}\right]$ there exists a symmetric admissible $w_{\varrho} \in C^{1,1}([-\varrho, \varrho],(0, \infty))$ with $w_{\varrho}( \pm \varrho)=u\left(x_{1}\right), w_{\varrho}^{\prime}(\varrho)=\alpha^{\prime}=u^{\prime}\left(x_{1}\right)$ and lower Willmore energy than $\left.u\right|_{\left[-x_{1}, x_{1}\right]}$. We choose $\varrho=u\left(x_{1}\right) x_{2} / u\left(x_{2}\right)$ so that we shorten the graph of $\left.u\right|_{\left[-x_{1}, x_{1}\right]}$ until the line $y \mapsto u\left(x_{2}\right) y / x_{2}$ is reached. By $\frac{u\left(x_{2}\right)}{x_{2}}>\frac{u\left(x_{1}\right)}{x_{1}}$ and $u\left(x_{2}\right)<\alpha^{*} x_{2}$ we see that indeed $\varrho \in\left[u\left(x_{1}\right) / \alpha^{*}, x_{1}\right]$. Moreover, since $u(x) \geq \alpha^{*} x$ in $[0, \bar{x}]$ and $u(x) / x$ is decreasing in $\left[\bar{x}, x_{1}\right]$ then by Proposition 4.42 , there exists $x^{\prime}$ such that $w_{\varrho}(x) \geq \alpha^{*} x$ in $\left[0, x^{\prime}\right]$ and $w_{\varrho}(x) / x$ is decreasing in $\left[x^{\prime}, \varrho\right]$. The function $v$ equal to $u$ in $\left[x_{2}, 1\right]$ and to the rescaled $w_{\varrho}$ in $\left[0, x_{2}\right]$ is admissible and has the same boundary values as $u$. Finally, $v$ satisfies (4.17) for some $\hat{x} \in[0,1)$ and there exists $\widetilde{x}$ such that $v(x) \geq \alpha^{*} x$ in $[0, \widetilde{x}]$ and $v(x)<\alpha^{*} x$ in $[\widetilde{x}, 1]$ and $v(x) / x$ has on $[\widetilde{x}, 1]$ at least two local extrema less than $u(x) / x$ in $[\bar{x}, 1]$.

Since $u(x) / x$ has only finitely many local extrema, the claim is proved by finitely many iterations.

Notice that if $u\left(x_{0}\right) \geq \mu x_{0}$ then also $v(\hat{x}) \geq \mu \hat{x}$.

The previous proposition is the main ingredient which allows us to pass to functions with uniformly bounded derivatives. For this purpose, we distinguish again the cases $\alpha \geq-\beta$ and $\alpha<-\beta$.

\section{The case $\alpha \geq-\beta$}

In the next lemma we prove that in the case $\alpha \geq-\beta$ it is sufficient to consider functions satisfying $u(x) \geq \alpha x$ in $[0,1]$.

Lemma 4.44. We assume in addition that $\alpha \geq-\beta$. Let $u \in N_{\alpha, \beta}$ be an admissible function in the sense of Definition 4.26 satisfying (4.17) for some $x_{0} \in[0,1)$ and $u^{\prime}(x) \neq-\beta$ for all $x \in[0,1)$ with $u(x) \leq \alpha x$.

Then, there exists an admissible function $v \in N_{\alpha, \beta}$ with lower Willmore energy than $u$, satisfying $v(x)>\alpha x$ for all $x \in[0,1)$ and (4.17) for some $x^{\prime} \in[0,1)$. 
Proof. We assume first that even $\alpha>-\beta$ so that $u(x)>\alpha x$ in a left neighbourhood of 1 . If $u(x) \leq \alpha x$ for some $x \in(0,1)$ then there exists a smallest element $x_{1}$ in $[0,1]$ such that $u\left(x_{1}\right)=\alpha x_{1}$. Let $x_{2} \geq x_{1}$ be the smallest element such that $u^{\prime}\left(x_{2}\right)=\alpha$ and $u\left(x_{2}\right) \leq \alpha x_{2}$. If $u(x) / x \geq u\left(x_{2}\right) / x_{2}$ for all $x \in\left(0, x_{2}\right]$ we denote $x_{2}$ by $\bar{x}$. Otherwise, $\bar{x}$ denotes the largest element in $\left(0, x_{2}\right)$ such that $u(x) / x \geq u(\bar{x}) / \bar{x}$ for all $x \in(0, \bar{x}]$. Then $u(\bar{x}) \leq \alpha \bar{x}, u^{\prime}(\bar{x}) \leq \alpha$ and, by assumption, $u^{\prime}(\bar{x})>-\beta$. Let $\widetilde{x} \in\left[x_{2}, 1\right]$ be the biggest element such that $u^{\prime}(\widetilde{x})=u^{\prime}(\bar{x})$ and $u(\widetilde{x})>\alpha \widetilde{x}$. Such an element exists since $-\beta<\alpha$.

We notice first that $u(\widetilde{x})<\alpha^{*} \widetilde{x}$. Indeed, if $u(\widetilde{x}) \geq \alpha^{*} \widetilde{x}$ since $u^{\prime}(\widetilde{x}) \leq \alpha<\alpha^{*}$ then $u(x)>\alpha^{*} x$ in a left neighbourhood of $\widetilde{x}$ and by Lemma 4.30 for these points $u^{\prime}(x)<\alpha^{*}$ and by continuity of $u$ and of its derivative then $u(x) \geq \alpha^{*} x$ for all $x \in[0, \tilde{x}]$. This contradicts the assumption that $u(x)<\alpha x$ in some interval.

The construction is now done similarly to Proposition 4.43. By using a scaled version of Proposition 4.42 we shorten the function $\left.u\right|_{[-\bar{x}, \bar{x}]}$ by inserting a cosh or an arc of a circle. We shorten it until we reach the line $y \mapsto u(\widetilde{x}) y / \widetilde{x}$. That is, we consider the function $w_{\varrho}$ with $\varrho=u(\bar{x}) \widetilde{x} / u(\widetilde{x})$ constructed by a rescaled version of Proposition 4.42 applied to $\left.u\right|_{[-\bar{x}, \bar{x}]}$. Since $u(x) / x \geq u(\bar{x}) / \bar{x}$ in $(0, \bar{x}]$ then by the first claim in Proposition 4.42, we have $w_{\varrho}(x) \geq w_{\varrho}(\varrho) x / \varrho$ in $(0, \varrho]$. Hence, the function $v$ which is equal to $u$ in $[\widetilde{x}, 1]$ and equal to the rescaled $w_{\varrho}$ in $[0, \widetilde{x}]$ yields the claim.

If $\alpha=-\beta$, let $\bar{x} \in(0,1]$ be the smallest element such that $v(\bar{x})=\alpha \bar{x}$. By the assumptions it follows that $\bar{x}=1$. Indeed, if $\bar{x}<1$ then, $u^{\prime}(\bar{x}) \leq \alpha=-\beta$ and the assumption gives $u^{\prime}(\bar{x})<-\beta=$ $\alpha$. But there exists then $x^{\prime}>\bar{x}$ such that $v^{\prime}\left(x^{\prime}\right)=\alpha=-\beta$ and $v\left(x^{\prime}\right)<\alpha x^{\prime}$, a contradiction.

We may now assume that $u(x) \geq \alpha x$ in $[0,1]$. In the next corollary, we first observe that the set $\left\{x \in[0,1]: \alpha x \leq u(x) \leq \alpha^{*} x\right\}$ is an interval and then, by using Proposition 4.43, we show that we may assume that in this interval $u(x) / x$ is decreasing. This yields suitable a priori bounds.

Corollary 4.45. Let $\alpha$ be such that $-\beta \leq \alpha$. Let $u \in N_{\alpha, \beta}$ be an admissible function in the sense of Definition 4.26 satisfying (4.17) for some $x_{0} \in[0,1), u^{\prime}(x) \neq-\beta$ for all $x \in[0,1)$ with $u(x) \leq \alpha x$.

Then, there exists an admissible function $v \in N_{\alpha, \beta}$ with lower Willmore energy than $u$ and satisfying $v^{\prime}(x) \leq \alpha^{*}$ in $[0,1]$ and $v(x)>\alpha x$ in $[0,1)$ as well as (4.17) for some $x_{1} \in[0,1)$.

Proof. By Lemmas 4.44 and 4.41 and the following remark, there exists $w \in N_{\alpha, \beta}$ with lower Willmore energy than $u$ such that $w(x)>\alpha x$ in $[0,1), w$ satisfies (4.17) for some $x^{\prime} \in[0,1)$ and so that there exists $\bar{x} \in(0,1)$ such that $w(x) \geq \alpha^{*} x$ in $[0, \bar{x}]$ and $w(x)<\alpha^{*} x$ in $(\bar{x}, 1]$. By Proposition 4.43 there exists $v \in N_{\alpha, \beta}$ with lower Willmore energy than $w$ such that $v$ satisfies (4.17) for some $x_{1} \in[0,1)$ and so that there exists $x_{2} \in(0,1)$ such that $v(x) \geq \alpha^{*} x$ in $\left[0, x_{2}\right]$ and $v(x) / x$ is decreasing in $\left[x_{2}, 1\right]$. This shows in particular that $v(x)>\alpha x$ in $[0,1)$. Since $v^{\prime}(x) \leq v(x) / x$ in $\left[x_{2}, 1\right]$ we find $v^{\prime}(x) \leq \alpha^{*}$ in $\left[x_{2}, 1\right]$. Reasoning as in Lemma 4.41 we get $v^{\prime}(x) \leq \alpha^{*}$ in $[0,1]$.

\section{The case $\alpha<-\beta$}

The next result corresponds to Corollary 4.45. We first observe that we have a bound on the derivative when the graph of $u$ is below the line $y \mapsto \alpha y$. Then, when the graph of $u$ crosses this line, we are back in the previous case.

Corollary 4.46. We assume in addition that $-\beta>\alpha$. Let $u \in N_{\alpha, \beta}$ be an admissible function in the sense of Definition 4.26 satisfying (4.17) for some $x_{0} \in[0,1)$ and $u^{\prime}(x) \neq-\beta$ for all $x \in[0,1)$ with $u(x) \leq \alpha x$.

Then, there exists an admissible function $v \in N_{\alpha, \beta}$ with lower Willmore energy than $u$, satisfying (4.17) for some $x_{1} \in[0,1)$ and $v^{\prime}(x) \leq \max \left\{-\beta, \alpha^{*}\right\}$ on $[0,1]$. 
Proof. Let $w \in N_{\alpha, \beta}$ be the function constructed in Lemma 4.41 such that $\mathcal{W}_{h}(w) \leq \mathcal{W}_{h}(u)$, $w$ satisfies $(4.17)$ for some $\widetilde{x} \in[0,1)$ and there exists $\bar{x} \in(0,1)$ with $w(x) \geq \alpha^{*} x$ in $[0, \bar{x}]$ and $w(x)<\alpha^{*} x$ in $(\bar{x}, 1]$. Notice that by the construction also $w$ satisfies that $w^{\prime}(x) \neq-\beta$ for all $x \in[0,1)$ with $w(x)<\alpha x$. Let $x_{2}$ be the biggest element in $(0,1)$ such that $w\left(x_{2}\right)=\alpha x_{2}$. Then for all $x \in\left(x_{2}, 1\right)$ we have $w(x)<\alpha x$ and $w^{\prime}(x)<-\beta$.

If $\widetilde{x} \geq x_{2}$ the claim follows with $v=w$ and $x_{1}=\widetilde{x}$. Otherwise, since $w\left(x_{2}\right)=\alpha x_{2}<\alpha^{*} x_{2}$ and $w^{\prime}\left(x_{2}\right) \leq \alpha$ then $w\left(x_{2}\right) / x_{2} \geq w^{\prime}\left(x_{2}\right)$. Hence, applying a rescaled version of Lemma 4.44 and of Corollary 4.45 to $\left.w\right|_{\left[-x_{2}, x_{2}\right]}$ we find an admissible function $v \in C^{1,1}\left(\left[-x_{2}, x_{2}\right],(0, \infty)\right)$ with lower Willmore energy than $\left.w\right|_{\left[-x_{2}, x_{2}\right]}$ with the same boundary values and such that $v(x)>\alpha x$ in $\left[0, x_{2}\right)$ and $v^{\prime}(x) \leq \alpha^{*}$ in $\left[0, x_{2}\right)$. Defining $v(x)=w(x)$ for $x \in\left[x_{2}, 1\right]$ and extending $v$ by symmetry to the interval $[-1,1]$ yields the claim.

\section{Characterisation of suitable minimising sequences}

The following proposition characterises suitably modified minimising sequences. We do not need to distinguish the cases $\alpha \geq-\beta$ and $\alpha<-\beta$.

Proposition 4.47. Let $\left(u_{k}\right)_{k \in \mathbb{N}}$ be a minimising sequence for $M_{\alpha, \beta}$ in $N_{\alpha, \beta}$ of admissible functions in the sense of Definition 4.26 such that $\mathcal{W}_{h}\left(u_{k}\right) \leq M_{\alpha, \beta}+1$ for all $k \in \mathbb{N}$. Then, there exists a minimising sequence $\left(v_{k}\right)_{k \in \mathbb{N}} \subset N_{\alpha, \beta}$ of admissible functions having lower Willmore energy and satisfying (4.17) as well as

$$
\max \left\{-\beta, \alpha^{*}\right\} \geq v_{k}^{\prime}(x) \geq-\frac{\operatorname{arsinh}(-\beta)}{\alpha}\left(\alpha_{\beta}-\alpha\right) \text { and } C_{\alpha, \beta} \leq v_{k}(x) \leq \sqrt{1+\alpha^{2}-x^{2}},
$$

in $[0,1]$ with a constant $C_{\alpha, \beta}>0$ depending on $\alpha,-\beta$ and $M_{\alpha, \beta}$.

Proof. By Lemmas 4.28, 4.29, 4.34, 4.44 and Corollary 4.45 if $-\beta \leq \alpha$ or Corollary 4.46 if $-\beta>\alpha$, for each $u_{k}$ there exists an admissible $v_{k} \in N_{\alpha, \beta}$ with lower Willmore energy than $u_{k}$ satisfying (4.17) for some $x_{k} \in[0,1)$ and

$$
v_{k}^{\prime}(x) \leq \max \left\{-\beta, \alpha^{*}\right\} \quad \text { and } \quad v_{k}\left(x_{k}\right) \geq \frac{\alpha}{\operatorname{arsinh}(-\beta)\left(\alpha_{\beta}-\alpha\right)} x_{k} .
$$

Since $v_{k}$ satisfies $(4.16)$ we get $v_{k}(x) \leq \sqrt{1+\alpha^{2}-x^{2}}$ for $x \in[0,1]$ and

$$
v_{k}^{\prime}(x) \geq-\frac{x}{v_{k}(x)} \geq-\frac{x_{k}}{v_{k}\left(x_{k}\right)} \geq-\operatorname{arsinh}(-\beta) \frac{\alpha_{\beta}-\alpha}{\alpha} \text { for } x \in\left[0, x_{k}\right],
$$

while $v_{k}^{\prime} \geq 0$ in $\left[x_{k}, 1\right]$. The estimate from below for $v_{k}$ follows from the second estimate in (4.23) if $x_{k} \geq 1 / 2$ and from Lemma 4.9 if $x_{k} \leq 1 / 2$.

\section{Proof of the existence theorem}

Theorem 4.48 (Existence and regularity). For $\beta<0$ and $\alpha<\alpha^{*}$ there exists a symmetric function $u \in C^{\infty}([-1,1],(0, \infty))$ such that the corresponding surface of revolution $\Gamma \subset \mathbb{R}^{3}$ solves the Dirichlet problem (1.4). This solution u has at most one critical point in $(0,1)$ and obeys the following estimates:

$$
\begin{gathered}
\max \left\{\alpha^{*},-\beta\right\} \geq u^{\prime}(x) \geq-\left(\alpha-\alpha_{\beta}\right) \frac{\operatorname{arsinh}(-\beta)}{\alpha} \text { in }(0,1] \\
\text { and } \sqrt{1+\alpha^{2}-x^{2}} \geq u(x) \geq C_{\alpha, \beta} \text { in }[-1,1],
\end{gathered}
$$

with a constant $C_{\alpha, \beta}>0$ depending on $M_{\alpha, \beta}, \alpha$ and $-\beta$. 
Proof. By density of polynomials in $H^{2}(-1,1)$ a minimising sequence $\left(u_{k}\right)_{k \in \mathbb{N}}$ for $M_{\alpha, \beta}$ may be chosen in $N_{\alpha, \beta}$ which consists of positive symmetric polynomials of degree at least 2 and obeys $\mathcal{W}_{h}\left(u_{k}\right) \leq M_{\alpha, \beta}+1$ for all $k \in \mathbb{N}$. By Proposition 4.47 , there exists a minimising sequence $\left(v_{k}\right)_{k \in \mathbb{N}} \subset N_{\alpha, \beta}$ such that $\mathcal{W}_{h}\left(v_{k}\right) \leq \mathcal{W}_{h}\left(u_{k}\right)$ and each element $v_{k}$ of the minimising sequence satisfies (4.22). The rest of the proof is along the lines of Theorem 3.11. Moreover, Lemma 3.20 shows that $u$ has at most one critical point in $(0,1)$.

Reasoning as in the proof of Propositions 3.12 and 4.18, one can prove that also in this case the energy is strictly decreasing in $\alpha$.

Proposition 4.49. Let $M_{\alpha, \beta}$ be as defined in (2.7) and $\alpha<\alpha^{*}$. Then $M_{\widetilde{\alpha}, \beta}>M_{\alpha, \beta}$ for all $\widetilde{\alpha}<\alpha$.

\section{Convergence to the sphere for $\alpha \searrow 0$}

In this section, we choose any $\beta \in \mathbb{R}$, keep it fixed and study the singular limit $\alpha \searrow 0$, where the "holes" $\{ \pm 1\} \times B_{\alpha}(0)$ in the cylindrical surfaces of revolution disappear.

The aim of this chapter is to show that if $u_{\alpha} \in N_{\alpha, \beta}$ is an energy minimising solution to (1.4), i.e. $\mathcal{W}_{h}\left(u_{\alpha}\right)=M_{\alpha, \beta}$, then $u_{\alpha}$ converges for $\alpha \searrow 0$ to the semicircle $\sqrt{1-x^{2}}$. So, the surface of revolution generated by the graph of $u_{\alpha}$ converges to the sphere, which shows up as a limit irrespective of the prescribed boundary slope $\pm \beta$.

We first show that for $\alpha$ small, any minimiser $u_{\alpha} \in N_{\alpha, \beta}$ of $\mathcal{W}_{h}$, i.e. $\mathcal{W}_{h}\left(u_{\alpha}\right)=M_{\alpha, \beta}$, has the same qualitative properties as the solution we have constructed.

Lemma 5.1. We assume that $\alpha<\min \left\{\alpha^{*}, 1 /|\beta|\right\}$. Let $u \in N_{\alpha, \beta}$ be such that $\mathcal{W}_{h}(u)=M_{\alpha, \beta}$. Then, $u \in C^{\infty}([-1,1],(0, \infty))$ and $u$ has the following additional properties:

1. If $\beta \geq 0$, then $u^{\prime}<0$ in $(0,1)$ and

$$
\alpha \leq u(x) \leq \sqrt{1+\alpha^{2}-x^{2}} \text { in }[-1,1], x+u(x) u^{\prime}(x)>0 \text { in }(0,1) .
$$

2. If $\beta<0$, then $u$ has at most one critical point in $(0,1)$, i.e. there exists $x_{0} \in[0,1)$ such that $u^{\prime}>0$ in $\left(x_{0}, 1\right], u^{\prime}\left(x_{0}\right)=0$ and $u^{\prime}<0$ in $\left(0, x_{0}\right)$. Moreover,

$$
\begin{gathered}
x+u(x) u^{\prime}(x)>0 \text { in }(0,1], \quad u^{\prime}(x) \leq \gamma:=\max \left\{-\beta, \alpha^{*}\right\} \text { in }\left[x_{0}, 1\right] \\
\text { and } u(x) \geq \min \left\{\frac{1}{2} \frac{\alpha}{\operatorname{arsinh}(-\beta)\left(\alpha_{\beta}-\alpha\right)}, \frac{1}{2} \frac{\gamma}{e^{C}-1}\right\} \text { in }[-1,1],
\end{gathered}
$$

with $C=\frac{\gamma \sqrt{1+\gamma^{2}}}{2}\left(M_{\alpha, \beta}+\frac{4 \beta}{\sqrt{1+\beta^{2}}}\right)>0$.

Proof. Since $u$ minimises $\mathcal{W}_{h}$ and hence, weakly solves the Euler-Lagrange equation (2.5), the argument in [DDG, Theorem 3.9, Step 2] yields $u \in C^{\infty}([-1,1],(0, \infty))$. Whenever $x_{0} \in(0,1)$ is a critical point of $u$ one may insert on $\left[-x_{0}, x_{0}\right]$ a rescaled energy minimising solution $v$ according to Theorem 3.18 and Lemma 3.20 satisfying $v^{\prime}<0$ on $\left(0, x_{0}\right)$. Putting together $v$ and $\left.u\right|_{[-1,1] \backslash\left[-x_{0}, x_{0}\right]}$ yields a further minimiser in $N_{\alpha, \beta}$ and so, a solution to (2.5). By uniqueness of the initial value problem, this new solution coincides with the original $u$. This shows that $u$ has at most one critical point. Exploiting this observation, one proves that $u$ satisfies the estimate in the claim by the same constructions as in the proof of the respective existence theorems.

The assumption on $\alpha$ excludes in particular the case $0<-\beta<\alpha$ and $\alpha>\alpha_{\beta}$, where "our" solution was constructed in a much smaller set than $N_{\alpha, \beta}$.

In what follows, when considering minimisers $u$ of $\mathcal{W}_{h}$ in $N_{\alpha, \beta}$, we make use of the qualitative properties of $u$ stated in Lemma 5.1 without further notice. In particular, we restrict ourselves always to the case $\alpha<\min \left\{\alpha^{*}, 1 /|\beta|\right\}$. 


\subsection{An upper bound for the energy as $\alpha \searrow 0$}

For $\alpha$ small we first construct a function $f_{\alpha} \in N_{\alpha, \beta}$ such that its Willmore energy converges to the one of the sphere for $\alpha \searrow 0$. We consider the symmetric function:

$$
f_{\alpha}(x)= \begin{cases}\frac{\alpha}{\sqrt{1+\beta^{2}}} \cosh \left(\frac{\sqrt{1+\beta^{2}}}{\alpha}\left(x-x_{1}\right)\right) & \text { if } x_{0} \leq x \leq 1, \\ \sqrt{r^{2}-x^{2}} & \text { if }-x_{0}<x<x_{0}, \\ \frac{\alpha}{\sqrt{1+\beta^{2}}} \cosh \left(\frac{\sqrt{1+\beta^{2}}}{\alpha}\left(x+x_{1}\right)\right) & \text { if }-1 \leq x \leq-x_{0},\end{cases}
$$

where $x_{1}=1-\alpha \operatorname{arsinh}(-\beta) / \sqrt{1+\beta^{2}}, r^{2}=x_{0}^{2}+f_{\alpha}\left(x_{0}\right)^{2}$ and, for $\alpha$ small enough, $x_{0} \in(0,1)$ is solution of

$$
-x_{0}=\frac{\alpha}{2 \sqrt{1+\beta^{2}}} \sinh \left(2 \frac{\sqrt{1+\beta^{2}}}{\alpha}\left(x_{0}-x_{1}\right)\right) .
$$

Assuming $\alpha$ to be small enough ensures the existence of $x_{0} \in(0,1)$. We remark that this $x_{0}$ should not be mixed with the one in Condition (4.17). The function $f_{\alpha}$ has Willmore energy

$$
\begin{aligned}
\mathcal{W}_{h}\left(f_{\alpha}\right) & =2 \int_{x_{0}}^{1} \kappa_{h}\left[f_{\alpha}\right]^{2} \frac{\sqrt{1+f_{\alpha}^{\prime 2}(x)}}{f_{\alpha}(x)} d x=8 \int_{x_{0}}^{1} \frac{1}{\cosh ^{2}\left(\frac{\sqrt{1+\beta^{2}}}{\alpha}\left(x-x_{1}\right)\right)} \frac{\sqrt{1+\beta^{2}}}{\alpha} d x \\
& =8 \tanh (\operatorname{arsinh}(-\beta))-8 \tanh \left(\frac{\sqrt{1+\beta^{2}}\left(x_{0}-x_{1}\right)}{\alpha}\right) \\
& =-8 \frac{\beta}{\sqrt{1+\beta^{2}}}+8 \tanh \left(\frac{\sqrt{1+\beta^{2}}\left(x_{1}-x_{0}\right)}{\alpha}\right) \leq-8 \frac{\beta}{\sqrt{1+\beta^{2}}}+8 .
\end{aligned}
$$

This particular function shows that $M_{\alpha, \beta}$ is uniformly bounded for $\alpha$ going to 0 . Since $M_{\alpha, \beta}$ is increasing for $\alpha \searrow 0$ for all $\beta \in \mathbb{R}$, it follows that

$$
\lim _{\alpha \searrow 0} M_{\alpha, \beta} \leq 8-8 \frac{\beta}{\sqrt{1+\beta^{2}}}
$$

\subsection{The limit of the energy}

In this section we prove that the limit of the energy is equal to the upper bound given in the previous section.

We start by proving that when $\beta<0$ and $\alpha$ is small the minimiser has precisely one critical point in $(0,1)$ and this point approaches 1 for $\alpha$ going to 0 .

Lemma 5.2. Let $\beta<0$. We assume that $u_{\alpha} \in N_{\alpha, \beta}$ minimises the Willmore energy, i.e. $\mathcal{W}_{h}\left(u_{\alpha}\right)=M_{\alpha, \beta}$. Let $x_{\alpha} \in[0,1)$ be such that $u_{\alpha}^{\prime}\left(x_{\alpha}\right)=0$ and $u_{\alpha}^{\prime}>0$ in $\left(x_{\alpha}, 1\right]$. Then,

$$
\lim _{\alpha \searrow 0} x_{\alpha}=1
$$

Proof. Let us assume that there exist a sequence $\alpha_{k} \searrow 0$ and $\delta>0$ such that $x_{\alpha_{k}} \leq 1-\delta$ for all $k \in \mathbb{N}$. For each $\alpha>0$, the measure of the set $A:=\left\{x \in[1-\delta, 1]: u_{\alpha}^{\prime}(x) \geq 2 \alpha / \delta\right\}$ is bounded by 
$\delta / 2$, since $u_{\alpha}$ is strictly positive. Then looking at the energy we find

$$
\begin{aligned}
& M_{\alpha_{k}, \beta}=\mathcal{W}_{h}\left(u_{\alpha_{k}}\right) \geq \int_{x_{\alpha_{k}}}^{1} \frac{1}{u_{\alpha_{k}}(x) \sqrt{1+u_{\alpha_{k}}^{\prime 2}(x)}} d x+\left.2 \frac{u_{\alpha_{k}}^{\prime}(x)}{\sqrt{1+u_{\alpha_{k}}^{\prime}(x)^{2}}}\right|_{-1} ^{1} \\
& \geq \int_{[1-\delta, 1] \backslash A} \frac{1}{\alpha_{k} \sqrt{1+u_{\alpha_{k}}^{\prime}(x)^{2}}} d x-4 \frac{\beta}{\sqrt{1+\beta^{2}}} \\
& \geq \frac{\delta}{2 \alpha_{k} \sqrt{1+\frac{4 \alpha_{k}^{2}}{\delta^{2}}}}-4 \frac{\beta}{\sqrt{1+\beta^{2}}} \rightarrow \infty \text { for } k \rightarrow \infty,
\end{aligned}
$$

a contradiction to (5.3).

We show that the gradient of any minimiser is unbounded near $x=-1$ in the limit $\alpha \searrow 0$. From now on, $\beta$ is a fixed element of $\mathbb{R}$.

Lemma 5.3. We fix $\delta_{0} \in(0,1)$. For $\alpha>0$, let $u_{\alpha} \in N_{\alpha, \beta}$ be a minimiser of the Willmore energy, i.e. $\mathcal{W}_{h}\left(u_{\alpha}\right)=M_{\alpha, \beta}$. Then,

$$
\lim _{\alpha \searrow 0} \max _{x \in\left[-1,-1+\delta_{0}\right]} u_{\alpha}^{\prime}(x)=+\infty .
$$

Proof. We assume by contradiction that there exist a sequence $\alpha_{k} \searrow 0$ and a positive constant $K$ such that

$$
\max _{x \in\left[-1,-1+\delta_{0}\right]} u_{\alpha_{k}}^{\prime}(x) \leq K \quad \text { for all } k \in \mathbb{N} .
$$

Let $x_{\alpha_{k}}=1$ if $\beta \geq 0$. If $\beta<0$, let $x_{\alpha_{k}} \in[0,1)$ be the element such that $u_{\alpha_{k}}^{\prime}\left(x_{\alpha_{k}}\right)=0$ and $u_{\alpha_{k}}^{\prime}>0$ in $\left(x_{\alpha_{k}}, 1\right]$. By Lemma 5.2 we have $1-x_{\alpha_{k}} \rightarrow 0$ for $k \rightarrow \infty$. Notice that $u_{\alpha_{k}}\left(x_{\alpha_{k}}\right) \leq \alpha_{k}$. Then, we estimate the Willmore energy from below as follows

$$
\begin{aligned}
\mathcal{W}_{h}\left(u_{\alpha_{k}}\right) & \geq \int_{-1}^{1} \frac{1}{u_{\alpha_{k}}(x)\left(1+u_{\alpha_{k}}^{\prime}(x)^{2}\right)^{\frac{1}{2}}} d x-4 \frac{\beta}{\sqrt{1+\beta^{2}}} \\
& \geq 2 \int_{-x_{\alpha_{k}}}^{-1+\delta_{0}} \frac{1}{u_{\alpha_{k}}(x)\left(1+u_{\alpha_{k}}^{\prime}(x)^{2}\right)^{\frac{1}{2}}} d x-4 \frac{\beta}{\sqrt{1+\beta^{2}}} .
\end{aligned}
$$

By (5.4) we have $u_{\alpha_{k}}(x) \leq u_{\alpha_{k}}\left(x_{\alpha_{k}}\right)+K(x+1)$ for $x \in\left[-x_{\alpha_{k}},-1+\delta_{0}\right]$ and hence from (5.5) we conclude for $k$ large enough

$$
\begin{aligned}
\mathcal{W}_{h}\left(u_{\alpha_{k}}\right) & \geq \frac{2}{\sqrt{1+K^{2}}} \int_{-x_{\alpha_{k}}}^{-1+\delta_{0}} \frac{1}{u_{\alpha_{k}}\left(x_{\alpha_{k}}\right)+K(x+1)} d x-4 \frac{\beta}{\sqrt{1+\beta^{2}}} \\
& =\frac{2}{K \sqrt{1+K^{2}}} \log \left(1+K \frac{\delta_{0}-1+x_{\alpha_{k}}}{u_{\alpha_{k}}\left(x_{\alpha_{k}}\right)+K\left(1-x_{\alpha_{k}}\right)}\right)-4 \frac{\beta}{\sqrt{1+\beta^{2}}} \\
& \geq \frac{2}{K \sqrt{1+K^{2}}} \log \left(1+\frac{K}{2} \frac{\delta_{0}}{u_{\alpha_{k}}\left(x_{\alpha_{k}}\right)+K\left(1-x_{\alpha_{k}}\right)}\right)-4 \frac{\beta}{\sqrt{1+\beta^{2}}}
\end{aligned}
$$

Since $u_{\alpha_{k}}\left(x_{\alpha_{k}}\right) \leq \alpha_{k} \searrow 0$ and $x_{\alpha_{k}} \rightarrow 1$ for $k \rightarrow \infty$, the energy $\mathcal{W}_{h}\left(u_{\alpha_{k}}\right)$ diverges to $+\infty$, thereby contradicting estimate (5.3). 
Theorem 5.4 (Limit of the energy for $\alpha \searrow 0$ ). For $\alpha>0$ let $u_{\alpha} \in N_{\alpha, \beta}$ be such that $\mathcal{W}_{h}\left(u_{\alpha}\right)=$ $M_{\alpha, \beta}$. Then, it holds that

$$
\lim _{\alpha \searrow 0} \mathcal{W}_{h}\left(u_{\alpha}\right)=\lim _{\alpha \searrow 0} M_{\alpha, \beta}=8-\frac{8 \beta}{\sqrt{1+\beta^{2}}}
$$

Proof. For any $u \in N_{\alpha, \beta}$ and $\delta_{0}>0$ to be chosen we have

$$
\begin{aligned}
\frac{1}{2} \mathcal{W}_{h}(u)= & \int_{-1}^{-1+\delta_{0}}\left(\frac{u^{\prime \prime}(x)}{\left(1+u^{\prime}(x)^{2}\right)^{\frac{3}{2}}}-\frac{1}{u(x)\left(1+u^{\prime}(x)^{2}\right)^{\frac{1}{2}}}\right)^{2} u(x) \sqrt{1+u^{\prime}(x)^{2}} d x \\
& +4 \int_{-1}^{-1+\delta_{0}} \frac{u^{\prime \prime}(x)}{\left(1+u^{\prime}(x)^{2}\right)^{\frac{3}{2}}} d x+\int_{-1+\delta_{0}}^{0} \kappa_{h}[u]^{2} \frac{\sqrt{1+u^{\prime}(x)^{2}}}{u(x)} d x \\
\geq & 4 \int_{-1}^{-1+\delta_{0}} \frac{u^{\prime \prime}(x)}{\left(1+u^{\prime}(x)^{2}\right)^{\frac{3}{2}}} d x=\left.4 \frac{u^{\prime}(x)}{\sqrt{1+u^{\prime}(x)^{2}}}\right|_{-1} ^{-1+\delta_{0}} \\
= & 4 \frac{u^{\prime}\left(-1+\delta_{0}\right)}{\sqrt{1+u^{\prime}\left(-1+\delta_{0}\right)^{2}}}-4 \frac{\beta}{\sqrt{1+\beta^{2}}} .
\end{aligned}
$$

Let $\alpha_{k} \searrow 0$ be any sequence. By Lemma 5.3 we find $\delta_{\alpha_{k}} \in[0,1 / 2]$ with $\lim _{k \rightarrow \infty} u_{\alpha_{k}}^{\prime}\left(-1+\delta_{\alpha_{k}}\right)=\infty$. From (5.6) it follows with $\delta_{0}=\delta_{\alpha_{k}}$

$$
\mathcal{W}_{h}\left(u_{\alpha_{k}}\right) \geq 8 \frac{u_{\alpha_{k}}^{\prime}\left(-1+\delta_{\alpha_{k}}\right)}{\sqrt{1+u_{\alpha_{k}}^{\prime}\left(-1+\delta_{\alpha_{k}}\right)^{2}}}-8 \frac{\beta}{\sqrt{1+\beta^{2}}}
$$

and hence

$$
\lim _{k \rightarrow \infty} \mathcal{W}_{h}\left(u_{\alpha_{k}}\right) \geq 8-8 \frac{\beta}{\sqrt{1+\beta^{2}}} .
$$

This estimate together with (5.3) yields the claim.

Corollary 5.5. For $\alpha>0$ let $u_{\alpha} \in N_{\alpha, \beta}$ be such that $\mathcal{W}_{h}\left(u_{\alpha}\right)=M_{\alpha, \beta}$. Then,

$$
\lim _{\alpha \searrow 0} \int_{-1+\delta_{0}}^{1-\delta_{0}} \kappa_{h}\left[u_{\alpha}\right]^{2} \frac{\sqrt{1+u_{\alpha}^{\prime}(x)^{2}}}{u_{\alpha}(x)} d x=0 \quad \text { for all } \delta_{0} \in(0,1) .
$$

Proof. For any sequence $\alpha_{k} \searrow 0$, by Lemma 5.3 there exist $\delta_{\alpha_{k}} \in\left[0, \delta_{0}\right]$ with

$$
\lim _{k \rightarrow \infty} u_{\alpha_{k}}^{\prime}\left(-1+\delta_{\alpha_{k}}\right)=+\infty
$$

Proceeding similarly as in the proof of Theorem 5.4 we have

$$
\mathcal{W}_{h}\left(u_{\alpha_{k}}\right) \geq \int_{-1+\delta_{\alpha_{k}}}^{1-\delta_{\alpha_{k}}} \kappa_{h}\left[u_{\alpha_{k}}\right]^{2} \frac{\sqrt{1+u_{\alpha_{k}}^{\prime}(x)^{2}}}{u_{\alpha_{k}}(x)} d x+8 \frac{u_{\alpha_{k}}^{\prime}\left(-1+\delta_{\alpha_{k}}\right)}{\sqrt{1+u_{\alpha_{k}}^{\prime}\left(-1+\delta_{\alpha_{k}}\right)^{2}}}-8 \frac{\beta}{\sqrt{1+\beta^{2}}} .
$$

Since

$$
0 \leq \int_{-1+\delta_{0}}^{1-\delta_{0}} \kappa_{h}\left[u_{\alpha_{k}}\right]^{2} \frac{\sqrt{1+u_{\alpha_{k}}^{\prime}(x)^{2}}}{u_{\alpha_{k}}(x)} d x \leq \int_{-1+\delta_{\alpha_{k}}}^{1-\delta_{\alpha_{k}}} \kappa_{h}\left[u_{\alpha_{k}}\right]^{2} \frac{\sqrt{1+u_{\alpha_{k}}^{\prime}(x)^{2}}}{u_{\alpha_{k}}(x)} d x
$$

the claim follows from the inequalities above, Theorem 5.4 and (5.7). 


\subsection{The minimiser converges to the sphere}

Lemma 5.6. Fix $\delta_{0} \in(0,1)$. For $\alpha>0$ let $u_{\alpha} \in N_{\alpha, \beta}$ solve $\mathcal{W}_{h}\left(u_{\alpha}\right)=M_{\alpha, \beta}$. Then, there exists $\varepsilon>0$ such that $u_{\alpha}(x) \geq \varepsilon$ in $\left[-1+\delta_{0}, 1-\delta_{0}\right]$ for all $\alpha \leq 1$.

Proof. We assume by contradiction that there is a sequence $1 \geq \alpha_{k} \searrow 0$ and that there are points $x_{k} \in\left[0,1-\delta_{0}\right]$ with $1 \geq u_{\alpha_{k}}\left(x_{k}\right)=\min _{x \in\left[0,1-\delta_{0}\right]} u_{\alpha_{k}}(x)=: m_{k} \searrow 0$. The energy of this sequence of minimisers is bounded from below as follows

$$
\begin{aligned}
\mathcal{W}_{h}\left(u_{\alpha_{k}}\right) & \geq \int_{-1}^{1} \frac{1}{u_{\alpha_{k}}(x) \sqrt{1+u_{\alpha_{k}}^{\prime}(x)^{2}}} d x-4 \frac{\beta}{\sqrt{1+\beta^{2}}} \\
& \geq 2 \int_{1-\delta_{0}}^{1} \frac{1}{u_{\alpha_{k}}(x) \sqrt{1+u_{\alpha_{k}}^{\prime}(x)^{2}}} d x-4 \frac{\beta}{\sqrt{1+\beta^{2}}} \\
& \geq \frac{2}{\max \left\{m_{k}, \alpha_{k}\right\}} \int_{1-\delta_{0}}^{1} \frac{1}{\sqrt{1+u_{\alpha_{k}}^{\prime}(x)^{2}}} d x-4 \frac{\beta}{\sqrt{1+\beta^{2}}} .
\end{aligned}
$$

In order to estimate the integral in (5.8), we apply the Cauchy-Schwarz inequality

$$
\begin{aligned}
\delta_{0} & =\int_{1-\delta_{0}}^{1} \frac{1}{\left(1+u_{\alpha_{k}}^{\prime}(x)^{2}\right)^{\frac{1}{4}}}\left(1+u_{\alpha_{k}}^{\prime}(x)^{2}\right)^{\frac{1}{4}} d x \\
& \leq\left(\int_{1-\delta_{0}}^{1} \frac{1}{\sqrt{1+u_{\alpha_{k}}^{\prime}(x)^{2}}} d x\right)^{\frac{1}{2}}\left(\int_{-\delta_{0}}^{1} \sqrt{1+u_{\alpha_{k}}^{\prime}(x)^{2}} d x\right)^{\frac{1}{2}},
\end{aligned}
$$

which implies

$$
\delta_{0}^{2} \leq\left(\delta_{0}+\int_{1-\delta_{0}}^{1}\left|u_{\alpha_{k}}^{\prime}(x)\right| d x\right) \int_{1-\delta_{0}}^{1} \frac{1}{\sqrt{1+u_{\alpha_{k}}^{\prime}(x)^{2}}} d x
$$

We estimate the first integral. Let $x_{\alpha_{k}}=1$ if $\beta \geq 0$ and if $\beta<0$ let $x_{\alpha_{k}} \in[0,1)$ be the element such that $u_{\alpha_{k}}^{\prime}\left(x_{\alpha_{k}}\right)=0$ and $u_{\alpha_{k}}^{\prime}>0$ in $\left(x_{\alpha_{k}}, 1\right]$. Lemma 5.2 shows that $x_{\alpha_{k}} \geq 1-\delta_{0}$ for sufficiently large $k$. Splitting the integral we find

$$
\int_{1-\delta_{0}}^{1}\left|u_{\alpha_{k}}^{\prime}(x)\right| d x \leq \int_{x_{k}}^{x_{\alpha_{k}}}\left|u_{\alpha_{k}}^{\prime}(x)\right| d x+\int_{x_{\alpha_{k}}}^{1}\left|u_{\alpha_{k}}^{\prime}(x)\right| d x= \begin{cases}m_{k}-\alpha_{k} & \text { if } \beta \geq 0, \\ m_{k}-2 u_{\alpha_{k}}\left(x_{\alpha_{k}}\right)+\alpha_{k} & \text { if } \beta<0 .\end{cases}
$$

Estimating the right hand side in the inequality above by $m_{k}+\alpha_{k}$, we then conclude from (5.9) that

$$
\delta_{0}^{2} \leq\left(\delta_{0}+m_{k}+\alpha_{k}\right) \int_{1-\delta_{0}}^{1} \frac{1}{\sqrt{1+u_{\alpha_{k}}^{\prime}(x)^{2}}} d x
$$

Inserting this into (5.8) yields for $k$ sufficiently large

$$
\begin{aligned}
\mathcal{W}_{h}\left(u_{\alpha_{k}}\right) & \geq \frac{2 \delta_{0}^{2}}{\max \left\{m_{k}, \alpha_{k}\right\}\left(\delta_{0}+m_{k}+\alpha_{k}\right)}-4 \frac{\beta}{\sqrt{1+\beta^{2}}} \\
& \geq \frac{2 \delta_{0}^{2}}{\max \left\{m_{k}, \alpha_{k}\right\}\left(\delta_{0}+2\right)}-4 \frac{\beta}{\sqrt{1+\beta^{2}}} \longrightarrow \infty \quad \text { for } k \rightarrow \infty,
\end{aligned}
$$


contradicting Theorem 5.4.

Corollary 5.7. Fix $\delta_{0} \in(0,1)$. For $\alpha>0$ small enough let $u_{\alpha} \in N_{\alpha, \beta}$ solve $\mathcal{W}_{h}\left(u_{\alpha}\right)=M_{\alpha, \beta}$. Then, there exists $\varepsilon>0$ such that

$$
-\frac{1}{\varepsilon} \leq u_{\alpha}^{\prime}(x) \leq \max \left\{\alpha^{*},-\beta\right\} \text { for all } x \in\left[0,1-\delta_{0}\right],
$$

where $\alpha^{*}=\min \{\cosh (b) / b: b>0\}$.

Proof. The first inequality is a consequence of Lemma 5.6 and the inequality $0 \leq x+u(x) u^{\prime}(x)$ in $[0,1]$. The second one follows from the estimates on the minimiser in Lemma 5.1.

Theorem 5.8 (Convergence to the sphere). For $\alpha>0$ sufficiently small let $u_{\alpha} \in N_{\alpha, \beta}$ be a minimiser of the Willmore energy, i.e. $\mathcal{W}_{h}\left(u_{\alpha}\right)=M_{\alpha, \beta}$. Let $u_{0}$ denote the semicircle $u_{0}(x):=$ $\sqrt{1-x^{2}}, x \in[-1,1]$. Then, for any $m \in \mathbb{N}$,

$$
\lim _{\alpha \searrow 0} u_{\alpha}=u_{0} \quad \text { in } C_{l o c}^{m}(-1,1) .
$$

Proof. We choose any $\delta_{0} \in(0,1)$. Let $\left(\alpha_{k}\right)_{k \in \mathbb{N}}$ be any sequence with $\alpha_{k} \searrow 0$. By Lemma 5.6 and Corollary 5.7 there exists a $\varepsilon>0$ such that

$$
\varepsilon \leq u_{\alpha_{k}}(x) \leq \sqrt{1+\alpha_{k}^{2}-x^{2}} \text { and } \quad-\frac{1}{\varepsilon} \leq u_{\alpha_{k}}^{\prime}(x) \leq \frac{1}{\varepsilon},
$$

for $x \in\left[-1+\delta_{0}, 1-\delta_{0}\right]$ and $k$ sufficiently large. By these uniform bounds, the monotonicity in $\alpha$ of the energy, and Theorem 5.4 we find

$$
\begin{aligned}
8-8 \frac{\beta}{\sqrt{1+\beta^{2}}} & \geq \mathcal{W}_{h}\left(u_{\alpha_{k}}\right) \\
& \geq \int_{-1+\delta_{0}}^{1-\delta_{0}} \frac{u_{\alpha_{k}}^{\prime \prime}(x)^{2} u_{\alpha_{k}}(x)}{\left(1+u_{\alpha_{k}}^{\prime}(x)^{2}\right)^{\frac{5}{2}}} d x+\int_{-1+\delta_{0}}^{1-\delta_{0}} \frac{1}{u_{\alpha_{k}}(x) \sqrt{1+u_{\alpha_{k}}^{\prime}(x)^{2}}} d x-4 \frac{\beta}{\sqrt{1+\beta^{2}}} \\
& \geq \frac{\varepsilon}{\left(1+\frac{1}{\varepsilon^{2}}\right)^{\frac{5}{2}}} \int_{-1+\delta_{0}}^{1-\delta_{0}} u_{\alpha_{k}}^{\prime \prime 2} d x+\frac{2}{\sqrt{1+\alpha_{k}^{2}}} \frac{1}{\left(1+\frac{1}{\varepsilon^{2}}\right)^{\frac{1}{2}}}-4 \frac{\beta}{\sqrt{1+\beta^{2}}} .
\end{aligned}
$$

Hence, $\left(u_{\alpha_{k}}\right)_{k \in \mathbb{N}}$ is uniformly bounded in $H^{2}\left(-1+\delta_{0}, 1-\delta_{0}\right)$. So, there exists a subsequence $\left(\alpha_{k_{j}}\right)_{j \in \mathbb{N}}$ and a function $\tilde{u}_{0} \in H^{2}\left(-1+\delta_{0}, 1-\delta_{0}\right)$ such that

$$
u_{\alpha_{k_{j}}}^{\prime \prime} \rightarrow \tilde{u}_{0}^{\prime \prime} \quad \text { in } L^{2}\left(-1+\delta_{0}, 1-\delta_{0}\right) \text { and } u_{\alpha_{k_{j}}} \rightarrow \tilde{u}_{0} \quad \text { in } C^{1}\left(\left[-1+\delta_{0}, 1-\delta_{0}\right],(0, \infty)\right) .
$$

Moreover, $\tilde{u}_{0}$ satisfies: $\varepsilon \leq \tilde{u}_{0}(x) \leq \sqrt{1-x^{2}},\left|\tilde{u}_{0}^{\prime}(x)\right| \leq \frac{1}{\varepsilon}$ for all $x \in\left[-1+\delta_{0}, 1-\delta_{0}\right]$ and by Corollary 5.5

$$
0=\liminf _{j \rightarrow \infty} \int_{-1+\delta_{0}}^{1-\delta_{0}} \kappa_{h}\left[u_{\alpha_{k_{j}}}\right]^{2} \frac{\sqrt{1+u_{\alpha_{k_{j}}}^{\prime}(x)^{2}}}{u_{\alpha_{k_{j}}}(x)} d x \geq \int_{-1+\delta_{0}}^{1-\delta_{0}} \kappa_{h}\left[\tilde{u}_{0}\right]^{2} \frac{\sqrt{1+\tilde{u}_{0}^{\prime}(x)^{2}}}{\tilde{u}_{0}(x)} d x .
$$

Hence, $\kappa_{h}\left[\tilde{u}_{0}\right] \equiv 0$ on $\left[-1+\delta_{0}, 1-\delta_{0}\right]$ and, therefore, $\left.\tilde{u}_{0}\right|_{\left[-1+\delta_{0}, 1-\delta_{0}\right]}$ is an arc of a geodesic circle, i.e., since $\tilde{u}_{0}$ is also symmetric around 0 , it exists a radius $r>0$ such that $\tilde{u}_{0}(x)=\sqrt{r^{2}-x^{2}}$ in 
$\left[-1+\delta_{0}, 1-\delta_{0}\right]$. Necessarily $r \geq 1-\delta_{0}$ and by the arbitrariness of $\delta_{0}$ we have $r \geq 1$. On the other hand, since $\tilde{u}_{0}(x) \leq \sqrt{1-x^{2}}$ we have $r \leq 1$. Hence, $r=1$ and $\tilde{u}_{0}=u_{0}$.

Since for any sequence $\left(\alpha_{k}\right)_{k \in \mathbb{N}}$ there exists a subsequence $\left(\alpha_{k_{j}}\right)_{j \in \mathbb{N}}$ such that $u_{\alpha_{k_{j}}}$ converges to $u_{0}$, we have that also $u_{\alpha_{k}}$ converges to $u_{0}$. The sequence being arbitrary, convergence in $C^{1} \cap H^{2}\left(\left[-1+\delta_{0}, 1-\delta_{0}\right]\right)$ follows. Proceeding as in the proof of regularity in [DDG, Theorem 3.9] we conclude from the weak form of the differential equation (2.5) that $u_{\alpha} \rightarrow u_{0}$ for $\alpha \searrow 0$ also in $C_{l o c}^{m}(-1,1)$ for any $m \in \mathbb{N}$.

\section{Qualitative properties of minimisers and estimates of the en- ergy}

In this section we give upper and lower bounds of the energy and we study the sign of the hyperbolic curvature of our minimiser. We have to distinguish the cases as in the proof of existence. We remark that any minimiser in the respective class of admissible functions has the qualitative properties mentioned in the existence theorems, since our geometric constructions apply also to these minimisers.

\subsection{The case $\alpha \beta>1$}

\subsubsection{Bounds on the energy}

Proposition 6.1 (Upper bound of the energy). We have

$$
M_{\alpha, \beta} \leq \frac{2(\alpha \beta-1)}{\sqrt{1+\beta^{2}}} \arcsin \frac{\beta}{\sqrt{1+\beta^{2}}} .
$$

Proof. We consider the arc $w \in N_{\alpha, \beta}$ of the circle with centre in $(0, \alpha-1 / \beta)$ and radius $\sqrt{1+\frac{1}{\beta^{2}}}$ which is given by

$$
w(x):=\alpha-\frac{1}{\beta}+\sqrt{1+\frac{1}{\beta^{2}}-x^{2}} \text { for } x \in[-1,1] .
$$

Its hyperbolic curvature is

$$
\kappa_{h}[w](x)=-\frac{\alpha \beta-1}{\sqrt{1+\beta^{2}}} \quad \text { for all } x \in[-1,1] .
$$

The previous identity for $\kappa_{h}[w]$ implies

$$
\begin{aligned}
\mathcal{W}_{h}(w) & =\frac{\alpha \beta-1}{\sqrt{1+\beta^{2}}} \int_{-1}^{1}\left(\frac{1}{\sqrt{1+\frac{1}{\beta^{2}}-x^{2}}}-\frac{1}{\alpha-\frac{1}{\beta}+\sqrt{1+\frac{1}{\beta^{2}}-x^{2}}}\right) d x \\
& \leq \frac{\alpha \beta-1}{\sqrt{1+\beta^{2}}} \int_{-1}^{1} \frac{1}{\sqrt{1+\frac{1}{\beta^{2}}-x^{2}}} d x
\end{aligned}
$$

The claim follows computing the integral.

The previous proposition indicates that possibly $\lim _{\alpha \rightarrow \infty} M_{\alpha, \beta}=\infty$. To prove this, we establish a lower bound for $M_{\alpha, \beta}$ for large $\alpha$. In what follows $G$ denotes the function $G: \mathbb{R} \rightarrow\left(-c_{0} / 2, c_{0} / 2\right)$,

$$
G(t)=\int_{0}^{t} \frac{1}{\left(1+\tau^{2}\right)^{\frac{5}{4}}} d \tau \text { with } c_{0}:=\int_{\mathbb{R}} \frac{1}{\left(1+\tau^{2}\right)^{\frac{5}{4}}} d \tau=\mathcal{B}(1 / 2,3 / 4)=2.39628 \ldots,
$$


with $\mathcal{B}(.,$.$) the Beta-Function. The G$-function played an important role in the study of the one-dimensional Willmore problem, i.e. of so called elastica, see [E, pp. 233-234] and [DG1, DG2].

The following estimate from below holds true for any $\beta \neq 0$, irrespective of its sign.

Proposition 6.2 (A lower bound for the energy). Let $\beta \in \mathbb{R} \backslash\{0\}$. For $\alpha>2|\beta|$ it holds that

$$
M_{\alpha, \beta} \geq \alpha \min \left\{G(-\beta)^{2},(G(\alpha / 2)+G(\beta))^{2}\right\}-4 \frac{\beta}{\sqrt{1+\beta^{2}}},
$$

where the function $G$ is defined in (6.2). In particular, $\lim _{\alpha \rightarrow \infty} M_{\alpha, \beta}=\infty$.

Proof. For any $u \in N_{\alpha, \beta}$ we have

$$
\mathcal{W}_{h}(u) \geq \int_{-1}^{1} \frac{u^{\prime \prime}(x)^{2} u(x)}{\left(1+u^{\prime}(x)^{2}\right)^{\frac{5}{2}}} d x-4 \frac{\beta}{\sqrt{1+\beta^{2}}}
$$

If $u(x) \geq \alpha / 2$ for all $x \in[-1,1]$ then

$$
\begin{aligned}
\mathcal{W}_{h}(u) & \geq \frac{\alpha}{2} \int_{-1}^{1} \frac{u^{\prime \prime}(x)^{2}}{\left(1+u^{\prime}(x)^{2}\right)^{\frac{5}{2}}} d x-4 \frac{\beta}{\sqrt{1+\beta^{2}}} \\
& \geq \frac{\alpha}{4}\left(\int_{-1}^{1} \frac{u^{\prime \prime}(x)}{\left(1+u^{\prime}(x)^{2}\right)^{\frac{5}{4}}} d x\right)^{2}-4 \frac{\beta}{\sqrt{1+\beta^{2}}} \\
& =\alpha(G(-\beta))^{2}-4 \frac{\beta}{\sqrt{1+\beta^{2}}} .
\end{aligned}
$$

Otherwise, there exists $x_{1} \in(0,1)$ such that $u(x) \geq \alpha / 2$ in $\left[x_{1}, 1\right]$ and $u^{\prime}\left(x_{1}\right) \geq \alpha / 2$. Proceeding similarly as before, Cauchy's inequality yields

$$
\begin{aligned}
\mathcal{W}_{h}(u) & \geq 2 \frac{\alpha}{2} \int_{x_{1}}^{1} \frac{u^{\prime \prime}(x)^{2}}{\left(1+u^{\prime}(x)^{2}\right)^{\frac{5}{2}}} d x-4 \frac{\beta}{\sqrt{1+\beta^{2}}} \\
& \geq \frac{\alpha}{1-x_{1}}\left(\int_{x_{1}}^{1} \frac{u^{\prime \prime}(x)}{\left(1+u^{\prime}(x)^{2}\right)^{\frac{5}{4}}} d x\right)^{2}-4 \frac{\beta}{\sqrt{1+\beta^{2}}} \\
& \geq \alpha(G(\alpha / 2)+G(\beta))^{2}-4 \frac{\beta}{\sqrt{1+\beta^{2}}} .
\end{aligned}
$$

One should observe that the function $G$ is strictly increasing. In both cases we have that

$$
\mathcal{W}_{h}(u) \geq \alpha \min \left\{G(\beta)^{2},(G(\alpha / 2)+G(\beta))^{2}\right\}-4 \frac{\beta}{\sqrt{1+\beta^{2}}}
$$

According to Propositions 6.1 and $6.2, M_{\alpha, \beta}$ grows linearly in $\alpha \rightarrow \infty$. We recall that for $\beta=0$ the situation is different since $M_{\alpha, 0} \rightarrow 0$, see [DDG, Lemma 3.2] and also Proposition 6.6 below. 


\subsubsection{On the sign of the hyperbolic curvature of minimisers}

From Proposition 3.7 we infer the following convexity property for minimisers of the Willmore functional.

Lemma 6.3. Let $u \in N_{\alpha, \beta}$ be a minimiser for $M_{\alpha, \beta}$. Then, $x \mapsto u^{\prime}(x)$ is strictly decreasing on $[0,1]$.

Proof. Since each minimiser satisfies $x+u(x) u^{\prime}(x)<0$ in $(0,1]$, the function $u^{\prime}$ is decreasing in a right neighbourhood of 0 . We assume by contradiction that $u^{\prime}$ is not strictly decreasing on $(0,1]$. Then, there exist $0<x_{1}<x_{2} \leq 1$ such that $u^{\prime}\left(x_{1}\right)=u^{\prime}\left(x_{2}\right)$. We consider $\left.u\right|_{\left[-x_{1}, x_{1}\right]}$ and rescale it to a function $w \in C^{1,1}\left(\left[-x_{2}, x_{2}\right],(0, \infty)\right)$. This function satisfies $w\left(x_{2}\right)=\frac{x_{2}}{x_{1}} u\left(x_{1}\right)>u\left(x_{1}\right) \geq u\left(x_{2}\right)$ and $w^{\prime}\left(x_{2}\right)=u^{\prime}\left(x_{2}\right)$. Moreover, by Remark 2.4 we conclude that

$$
\int_{-x_{2}}^{x_{2}} \kappa_{h}[w]^{2} d s_{h}[w]=\int_{-x_{1}}^{x_{1}} \kappa_{h}[u]^{2} d s_{h}[u]<\int_{-x_{2}}^{x_{2}} \kappa_{h}[u]^{2} d s_{h}[u],
$$

since $\kappa_{h}[u]$ is not identically zero in $\left[x_{1}, x_{2}\right]$. This follows since $u$ solves the differential equation (2.5) and is not part of a geodesic circle. On the other hand, exploiting that $u$ is a minimiser, we find

$$
\begin{aligned}
& \int_{-x_{2}}^{x_{2}} \kappa_{h}[u]^{2} d s_{h}[u]=\inf \left\{\int_{-x_{2}}^{x_{2}} \kappa_{h}[v]^{2} d s_{h}[v]: v \in C^{1,1}\left(\left[-x_{2}, x_{2}\right],(0, \infty)\right),\right. \text { symmetric, } \\
&\left.v\left(x_{2}\right)=u\left(x_{2}\right) \text { and } v^{\prime}\left(x_{2}\right)=u^{\prime}\left(x_{2}\right)\right\} \\
& \leq \int_{-x_{2}}^{x_{2}} \kappa_{h}[w]^{2} d s_{h}[w],
\end{aligned}
$$

where in the last step we used that $w\left(x_{2}\right)>u\left(x_{2}\right)$ and Proposition 3.7. One should observe that the condition $0>x+u(x) u^{\prime}(x)$ is the rescaled version of $\alpha \beta>1$ on the interval $[0, x]$. We have achieved a contradiction to (6.3).

Theorem 6.4. We assume that $\alpha \beta>1$. Let $u \in N_{\alpha, \beta}$ be a minimiser for $M_{\alpha, \beta}$. Then, either $\kappa_{h}[u]<0$ in $[0,1)$, or there exists $a \in(0,1)$ such that $\kappa_{h}[u]<0$ in $[0, a)$ and $\kappa_{h}[u]>0$ in $(a, 1)$.

Proof. We consider the auxiliary function $\varphi:[0,1] \rightarrow \mathbb{R}$ defined by $\varphi(x):=x+u(x) u^{\prime}(x)$, where $(\varphi(x), 0)$ is the centre and $r(x):=u(x) \sqrt{1+u^{\prime}(x)^{2}}, x \in[0,1]$ the radius of the geodesic circle being tangential to the graph of $u$ in $(x, u(x))$. From $(3.2)$ we know that $\varphi(x)<0$ in $(0,1]$, and $\varphi(0)=0$. Hence, $\varphi(x)$ is decreasing for $x>0$ sufficiently small. Since $\varphi^{\prime}(x)=\left\{1+u^{\prime}(x)^{2}\right\}^{\frac{3}{2}} \kappa_{h}[u](x)$, it follows that $\kappa_{h}[u]<0$ in a right neighbourhood of 0 . Viewing at (2.5) now as a second order equation for $\kappa_{h}[u]$ satisfying a strong maximum / minimum principle provided that maxima / minima are equal to zero yields that it suffices to show that $\kappa_{h}[u]$ has at most one sign change in $[0,1)$.

We assume by contradiction that there exist $0<x_{1}<x_{0}<x_{2} \leq 1$ such that $\kappa_{h}[u]>0$ in $\left(x_{1}, x_{0}\right), \kappa_{h}[u]<0$ in $\left(x_{0}, x_{2}\right)$ and $\varphi\left(x_{1}\right)=\varphi\left(x_{2}\right)$. We construct a new function with lower Willmore energy. This new function equals the original one on $\left[0, x_{1}\right]$. Then we take the arc of the circle with centre $\left(\varphi\left(x_{1}\right), 0\right)$ and radius $r\left(x_{1}\right)$, starting at $\left(x_{1}, u\left(x_{1}\right)\right)$ and ending where this arc intersects the straight line which connects $\left(\varphi\left(x_{1}\right), 0\right)=\left(\varphi\left(x_{2}\right), 0\right)$ and $\left(x_{2}, u\left(x_{2}\right)\right)$. Finally, we attach the suitably 
rescaled original function $\left.u\right|_{\left[x_{2}, 1\right]}$. More precisely, with the scaling factor $\varrho=r\left(x_{1}\right) / r\left(x_{2}\right)$ the new function is

$$
v(x):= \begin{cases}u(x) & \text { if } 0 \leq x \leq x_{1}, \\ \sqrt{r\left(x_{1}\right)^{2}-\left(x-\varphi\left(x_{1}\right)\right)^{2}} & \text { if } x_{1} \leq x \leq x_{2}+u^{\prime}\left(x_{2}\right) u\left(x_{2}\right)(1-\varrho), \\ \varrho u\left(\frac{1}{\varrho}\left(x-(1-\varrho) \varphi\left(x_{2}\right)\right)\right) & \text { if } x_{2}+u^{\prime}\left(x_{2}\right) u\left(x_{2}\right)(1-\varrho) \leq x \leq \varrho+(1-\varrho) \varphi\left(x_{2}\right),\end{cases}
$$

and extended by symmetry to $[-\ell(\varrho), \ell(\varrho)]$, setting $\ell(\varrho):=\varrho+(1-\varrho) \varphi\left(x_{2}\right)$. See Figure 13 . The function $v$ satisfies $v \in C^{1,1}([-\ell(\varrho), \ell(\varrho)],(0, \infty)), v(\ell(\varrho))=\varrho \alpha$ and $v^{\prime}(\ell(\varrho))=-\beta$.

a)

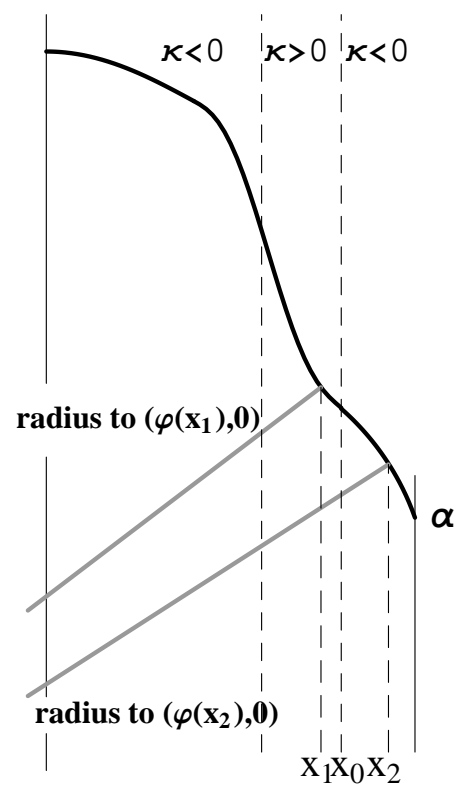

Figure 13: Proof of Theorem 6.4.

Note that $\varrho \leq 1$. Indeed, one may write

$$
r\left(x_{2}\right)=r\left(x_{1}\right)+\int_{x_{1}}^{x_{2}} \frac{u^{\prime}(t)}{\sqrt{1+u^{\prime}(t)^{2}}} \varphi^{\prime}(t) d t .
$$

By our assumption on $\varphi$ and since $u^{\prime}(x) \leq 0$ in $[0,1]$, the integrand in (6.5) is negative in $\left[x_{1}, x_{0}\right]$ and positive in $\left[x_{0}, x_{2}\right]$. Moreover, since $p \mapsto \frac{p}{\sqrt{1+p^{2}}}$ is strictly increasing and, by Lemma $6.3, u^{\prime}$ is strictly decreasing in $[0,1]$, also $\frac{u^{\prime}}{\sqrt{1+u^{\prime 2}}}$ is monotonically decreasing. Hence, splitting the integral in (6.5) and using that $\varphi\left(x_{1}\right)=\varphi\left(x_{2}\right)$ we get

$$
r\left(x_{2}\right)>r\left(x_{1}\right)+\frac{u^{\prime}\left(x_{0}\right)}{\sqrt{1+u^{\prime}\left(x_{0}\right)^{2}}} \int_{x_{1}}^{x_{0}} \varphi^{\prime}(t) d t+\frac{u^{\prime}\left(x_{0}\right)}{\sqrt{1+u^{\prime}\left(x_{0}\right)^{2}}} \int_{x_{0}}^{x_{2}} \varphi^{\prime}(t) d t=r\left(x_{1}\right) .
$$

By scaling, the function $w(x)=\frac{1}{\ell(\varrho)} v(\ell(\varrho) x)$ defined in $[-1,1]$, satisfies

$$
w^{\prime}(1)=-\beta, \quad w(1)=\frac{\varrho \alpha}{\ell(\varrho)} \quad \text { and } \quad \mathcal{W}_{h}(w)<\mathcal{W}_{h}(u)
$$


On the other hand, $\varrho<1, \varphi\left(x_{2}\right)<0$ give $\varrho>l(\varrho)$, and so $w(1)>\alpha=u(1)$. By monotonicity of the hyperbolic Willmore energy (see Proposition 3.12) we conclude that $\mathcal{W}_{h}(w)>\mathcal{W}_{h}(u)$. This contradicts (6.6).

\subsection{The case $\alpha \beta<1$ and $\beta \geq 0$}

\subsubsection{Bounds on the energy}

Proposition 6.5 (Upper bound for the energy). We have

$$
M_{\alpha, \beta} \leq \min \left\{\frac{2(1-\alpha \beta)}{\sqrt{1+\beta^{2}}} \frac{1}{\alpha}, 8 \tanh \left(\frac{\sqrt{1+\beta^{2}}}{\alpha}+\operatorname{arsinh}(\beta)\right)-8 \frac{\beta}{\sqrt{1+\beta^{2}}}\right\} .
$$

Proof. For $\beta>0$, the first estimate follows with the same construction as in Proposition 6.1 considering the function $w \in N_{\alpha, \beta}$ given by $(6.1) ; w(x)=\alpha-\frac{1}{\beta}+\sqrt{1+\frac{1}{\beta^{2}}-x^{2}}$. Computing its Willmore energy we find

$$
\begin{aligned}
\mathcal{W}_{h}(w) & =\frac{\alpha \beta-1}{\sqrt{1+\beta^{2}}} \int_{-1}^{1}\left(\frac{1}{\sqrt{1+\frac{1}{\beta^{2}}-x^{2}}}-\frac{1}{\alpha-\frac{1}{\beta}+\sqrt{1+\frac{1}{\beta^{2}}-x^{2}}}\right) d x \\
& \leq \frac{1-\alpha \beta}{\sqrt{1+\beta^{2}}} \int_{-1}^{1} \frac{1}{\alpha-\frac{1}{\beta}+\sqrt{1+\frac{1}{\beta^{2}}-x^{2}}} d x \leq \frac{1-\alpha \beta}{\sqrt{1+\beta^{2}}} \frac{2}{\alpha} .
\end{aligned}
$$

If $\beta=0$, the function $w(x) \equiv \alpha$ directly gives $M_{\alpha, 0} \leq \frac{2}{\alpha}$. Let $f_{\alpha}$ the function defined in (5.1). Notice that it is well defined since we assume that $\beta \geq 0$ and $\alpha \beta<1$. From the calculations on p. 44 we see that

$$
M_{\alpha, \beta} \leq \mathcal{W}_{h}\left(f_{\alpha}\right) \leq 8 \tanh \left(\frac{\sqrt{1+\beta^{2}}}{\alpha} x_{1}\right)-8 \frac{\beta}{\sqrt{1+\beta^{2}}}
$$

where $x_{1}=1+\frac{\alpha}{\sqrt{1+\beta^{2}}} \operatorname{arsinh}(\beta)$.

In the special case $\beta=0$ we may now characterise the asymptotic behaviour of $M_{\alpha, 0}$ for $\alpha \rightarrow \infty$. This is completely different from the case $\beta \neq 0$, cf. Proposition 6.2.

Proposition 6.6. We assume that $\alpha>0$ and $\beta=0$. Then, one has

$$
\frac{2 \alpha}{(\alpha+1) \sqrt{1+\alpha^{2}}} \leq M_{\alpha, 0} \leq 8 \tanh \left(\frac{1}{\alpha}\right)
$$

Proof. Let $u_{\alpha} \in N_{\alpha, 0}$ be a minimiser for $\mathcal{W}_{h}$ in $N_{\alpha, 0}$ as constructed in Theorem 3.18. It satisfies $u_{\alpha}(x) \leq \alpha+1$ and $\left|u_{\alpha}^{\prime}(x)\right| \leq 1 / \alpha$, which yields

$$
M_{\alpha, 0}=\mathcal{W}_{h}\left(u_{\alpha}\right) \geq 2 \int_{0}^{1} \frac{1}{u_{\alpha}(x) \sqrt{1+u_{\alpha}^{\prime}(x)^{2}}} d x \geq \frac{2 \alpha}{(\alpha+1) \sqrt{1+\alpha^{2}}},
$$

which is the estimate from below. The estimate from above was just proved in Proposition 6.5. 


\subsubsection{On the sign of the hyperbolic curvature of minimisers}

Theorem 6.7. We assume that $\beta \geq 0$ and that $\alpha \beta<1$. Let $u \in N_{\alpha, \beta}$ be a minimiser for $M_{\alpha, \beta}$. Then, $\kappa_{h}[u]>0$ in $(-1,1)$.

Proof. The proof is along the lines of Theorem 6.4. We recall the main points and emphasise on what is different. We associate to $u$ the auxiliary function $\varphi(x):=x+u(x) u^{\prime}(x), x \in[-1,1]$. From (3.4) we know that $\varphi(0)=0$ and $\varphi(x)>0$ in $(0,1]$. Since $\varphi$ cannot be constant in an interval, $\varphi(x)$ increases in a right neighbourhood of 0 and hence $\kappa_{h}[u](x)>0$ in a right neighbourhood of 0 .

We now prove that $\varphi^{\prime} \geq 0$ in $[0,1]$. If $\varphi^{\prime}<0$ in some interval then there exist points $0<$ $x_{0}<x^{\prime}<1$ such that $\varphi^{\prime} \geq 0$ (i.e. $\kappa_{h}[u] \geq 0$ ) in $\left[0, x_{0}\right]$ and $\varphi^{\prime}<0$ (i.e. $\kappa_{h}[u]<0$ ) in $\left(x_{0}, x^{\prime}\right.$ ). In particular, $u^{\prime \prime}<0$ in $\left[x_{0}, x^{\prime}\right]$. Let $x^{*} \in\left[0, x_{0}\right)$ be such that $u^{\prime \prime}(x)<0$ in $\left(x^{*}, x^{\prime}\right]$. Then, there exist $x_{1} \in\left[x^{*}, x_{0}\right)$ and $x_{2} \in\left(x_{0}, x^{\prime}\right]$ such that $\varphi\left(x_{1}\right)=\varphi\left(x_{2}\right)$.

Then, with the same notation as in the proof of Theorem 6.4, we consider the function $v \in$ $C^{1,1}([-\ell(\varrho), \ell(\varrho)],(0, \infty))$ defined as in (6.4). Then, $w(x):=\frac{1}{\ell(\varrho)} v(\ell(\varrho) x), x \in[-1,1]$, satisfies

$$
w^{\prime}(1)=-\beta, \quad w(1)=\frac{\varrho \alpha}{\ell(\varrho)} \quad \text { and } \quad \mathcal{W}_{h}(w)<\mathcal{W}_{h}(u) .
$$

Since $u^{\prime \prime}<0$ in $\left[x_{1}, x_{2}\right]$ and proceeding as in $(6.5)$ one sees that $\varrho<1$. Here, in contrast with the proof of Theorem $6.4, \varphi>0$ in $(0,1)$ and so $\varrho<\ell(\varrho)$. Hence, $w(1)<u(1)$ and $\mathcal{W}_{h}(w)>\mathcal{W}_{h}(u)$ by the strict monotonicity of the energy (Proposition 3.19), which contradicts the inequality in (6.7). Then, $\varphi$ being increasing in $[0,1]$ implies that $\kappa_{h}[u] \geq 0$ in $[-1,1]$. The strong minimum principle for $(2.5)$ considered as a second order equation for $\kappa_{h}[u]$ and applied to a possible minimum 0 yields that $\kappa_{h}[u]>0$ in $(-1,1)$.

\subsection{The case $\beta<0$ and $\alpha \geq \alpha_{\beta}$}

\subsubsection{Bounds on the energy}

Proposition 6.8 (Upper bound of the energy). We have

$$
M_{\alpha, \beta} \leq\left(1+\operatorname{arsinh}(-\beta)\left(\alpha-\alpha_{\beta}\right)\right) \frac{(-8 \beta)}{\sqrt{1+\beta^{2}}} .
$$

Proof. If $\alpha=\alpha_{\beta}$, the minimiser is $u_{c}(x)=\cosh (b x) / b, b=\operatorname{arsinh}(-\beta)$, which has vanishing mean curvature in $[-1,1]$ and hyperbolic Willmore energy

$$
\mathcal{W}_{h}\left(u_{c}\right)=-\frac{8 \beta}{\sqrt{1+\beta^{2}}} .
$$

If instead $\alpha>\alpha_{\beta}$, we consider the function $u:=u_{c}+\delta_{\alpha}$ where $\delta_{\alpha}:=\alpha-\alpha_{\beta}>0$. Notice that $u \in N_{\alpha, \beta}$. Since $u_{c} \geq \frac{1}{b}>0$ and $u_{c}^{\prime \prime}>0$ we have

$$
\begin{aligned}
\mathcal{W}_{h}\left(u_{c}+\delta_{\alpha}\right) & =\int_{-1}^{1}\left(\frac{u_{c}^{\prime \prime}}{\left(1+u_{c}^{\prime 2}\right)^{\frac{3}{2}}}+\frac{1}{\left(u_{c}+\delta_{\alpha}\right) \sqrt{1+u_{c}^{\prime 2}}}\right)^{2}\left(u_{c}+\delta_{\alpha}\right) \sqrt{1+u_{c}^{\prime 2}} d x \\
& \leq \mathcal{W}_{h}\left(u_{c}\right)+\int_{-1}^{1}\left(\frac{u_{c}^{\prime \prime}}{\left(1+u_{c}^{\prime 2}\right)^{\frac{3}{2}}}+\frac{1}{u_{c} \sqrt{1+u_{c}^{\prime 2}}}\right)^{2} \delta_{\alpha} \sqrt{1+u_{c}^{\prime 2}} d x \\
& \leq \mathcal{W}_{h}\left(u_{c}\right)+b \delta_{\alpha} \int_{-1}^{1}\left(\frac{u_{c}^{\prime \prime}}{\left(1+u_{c}^{\prime 2}\right)^{\frac{3}{2}}}+\frac{1}{u_{c} \sqrt{1+u_{c}^{\prime 2}}}\right)^{2} u_{c} \sqrt{1+u_{c}^{\prime 2}} d x
\end{aligned}
$$


which proves the proposition.

The previous result together with Proposition 6.2 shows that also for $\beta<0, M_{\alpha, \beta}$ grows linearly for $\alpha \rightarrow \infty$.

\subsubsection{On the sign of the hyperbolic curvature of minimisers}

Here we prefer a slightly less general formulation of the curvature statement and refer only to solutions as we have constructed. The reason is that we have to restrict the set of admissible functions in the case $-\beta<\alpha$.

Theorem 6.9. We assume that $\beta<0$ and that $\alpha \geq \alpha_{\beta}$. Let $u \in N_{\alpha, \beta}$ be an energy minimising solution of (1.4) as constructed in the proofs of Theorems 4.17 and 4.24. Then, either $\kappa_{h}[u]>0$ in $[0,1)$, or there exists a point $a \in(0,1)$ such that $\kappa_{h}[u]>0$ in $[0, a)$ and $\kappa_{h}[u]<0$ in $(a, 1)$.

Proof. We have $u^{\prime}>0$ in $(0,1]$. We associate to $u$ the function $\varphi(x):=x+u(x) u^{\prime}(x), x \in[-1,1]$, which satisfies $\varphi(0)=0$ and $\varphi(x)>0$ in $(0,1]$. Hence, $\varphi(x)$ increases in a right neighbourhood of 0 and so, $\kappa_{h}[u](x)>0$ in a right neighbourhood of 0 . In view of the strong maximum /minimum principle for (2.5) as a second order equation for $\kappa_{h}[u]$ applied to maximum /minimum equal to 0 , we only need to exclude that there is a sign change from $\kappa_{h}[u]<0$ to $\kappa_{h}[u]>0$.

We assume by contradiction that there exist $0<\tilde{x}<x_{0}<x^{\prime}$ such that $\kappa_{h}[u]<0$ (i.e. $\varphi^{\prime}<0$ ) in $\left(\tilde{x}, x_{0}\right)$ and $\kappa_{h}[u]>0$ (i.e. $\left.\varphi^{\prime}>0\right)$ in $\left(x_{0}, x^{\prime}\right)$. In particular, $u^{\prime \prime}<0$ in $\left[\tilde{x}, x_{0}\right]$ and so, also on a slightly larger interval $\left[\tilde{x}, x^{\prime \prime}\right) \supset\left[\tilde{x}, x_{0}\right]$. Then, we find $x_{1} \in\left(\tilde{x}, x_{0}\right)$ and $x_{2} \in\left(x_{0}, x^{\prime \prime}\right)$ with $\varphi\left(x_{1}\right)=\varphi\left(x_{2}\right)$.

We consider the function $v \in C^{1,1}([-\ell(\varrho), \ell(\varrho)],(0, \infty))$ as defined in (6.4). Using the same notation as in the proof of Theorem 6.4, one should notice that in this case $\varrho>1$. Indeed, one starts from (6.5). By our assumption on $\varphi$ and since $u^{\prime}(x)>0$ in $(0,1]$, the integrand in (6.5) is strictly negative in $\left(x_{1}, x_{0}\right)$ and positive in $\left(x_{0}, x_{2}\right)$. Moreover since $u^{\prime}$ is decreasing in $\left(x_{1}, x_{2}\right)$, also $\frac{u^{\prime}}{\sqrt{1+u^{\prime 2}}}$ is monotonically decreasing. Hence, splitting the integral in (6.5) and using that $\varphi\left(x_{1}\right)=\varphi\left(x_{2}\right)$ we get

$$
r\left(x_{2}\right)<r\left(x_{1}\right)+\frac{u^{\prime}\left(x_{0}\right)}{\sqrt{1+u^{\prime}\left(x_{0}\right)^{2}}} \int_{x_{1}}^{x_{0}} \varphi^{\prime}(t) d t+\frac{u^{\prime}\left(x_{0}\right)}{\sqrt{1+u^{\prime}\left(x_{0}\right)^{2}}} \int_{x_{0}}^{x_{2}} \varphi^{\prime}(t) d t=r\left(x_{1}\right) .
$$

Notice that even though $\varrho>1, x_{2}+u\left(x_{2}\right) u^{\prime}\left(x_{2}\right)(1-\varrho) \geq x_{1}$.

By scaling, the function $w(x)=\frac{1}{\ell(\varrho)} v(\ell(\varrho) x)$ defined in $[-1,1]$ satisfies

$$
w^{\prime}(1)=-\beta, \quad w(1)=\frac{\varrho \alpha}{\ell(\varrho)} \quad \text { and } \quad \mathcal{W}_{h}(w)<\mathcal{W}_{h}(u)
$$

On the other hand, since $\varphi>0$ on $(0,1]$ we have $\varrho>l(\varrho), w(1)>u(1)$ and hence $\mathcal{W}_{h}(w) \geq$ $\mathcal{W}_{h}(u)$ by monotonicity of the hyperbolic Willmore energy (see Propositions 4.18 and 4.25 ), which contradicts the inequality in (6.8).

\subsection{The case $\beta<0$ and $\alpha<\alpha_{\beta}$}

\subsubsection{Bounds on the energy}

Proposition 6.10 (Upper bound of the energy). The following estimate holds

$$
M_{\alpha, \beta} \leq-\frac{8 \beta}{\sqrt{1+\beta^{2}}}+8 \tanh \left(\frac{\operatorname{arsinh}(-\beta)}{\alpha}\left(\alpha_{\beta}-\alpha\right)\right) .
$$


Proof. For $\beta<0$ and $\alpha<\alpha_{\beta}$ the function $f_{\alpha}$ defined in (5.1) is well defined. As usual we denote $b=\operatorname{arsinh}(-\beta)$. The claim follows then from

$$
\begin{aligned}
M_{\alpha, \beta} & \leq \mathcal{W}_{h}\left(f_{\alpha}\right)=-\frac{8 \beta}{\sqrt{1+\beta^{2}}}-8 \tanh \left(\frac{b \alpha_{\beta}}{\alpha}\left(x_{0}-1+\frac{\alpha}{\alpha_{\beta}}\right)\right) \\
& \leq-\frac{8 \beta}{\sqrt{1+\beta^{2}}}+8 \tanh \left(\frac{b \alpha_{\beta}}{\alpha}\left(1-\frac{\alpha}{\alpha_{\beta}}\right)\right) .
\end{aligned}
$$

\subsubsection{On the sign of the hyperbolic curvature of minimisers}

Theorem 6.11. Let $u \in N_{\alpha, \beta}$ be a minimiser for $M_{\alpha, \beta}$. Then, $\kappa_{h}[u]>0$ in $(-1,1)$.

Proof. We know that there exists $x_{0} \in[0,1)$ such that $u^{\prime}\left(x_{0}\right)=0, u^{\prime}>0$ in $\left(x_{0}, 1\right]$ and $u^{\prime}<0$ in $\left(0, x_{0}\right)$. Then, $\left.u\right|_{\left[-x_{0}, x_{0}\right]}$ is the rescaled minimiser of $M_{u\left(x_{0}\right) / x_{0}, 0}$ and hence, by Theorem 6.7, $\kappa_{h}[u]>0$ in $\left(-x_{0}, x_{0}\right)$.

It remains to study the sign of the curvature in $\left[x_{0}, 1\right]$. Again, we consider $\varphi(x):=x+u(x) u^{\prime}(x)$, $x \in\left[x_{0}, 1\right]$. Since $u^{\prime}>0$ in $\left(x_{0}, 1\right], \varphi\left(x_{0}\right)=x_{0}$ and $\varphi(x)>x_{0}$ in $\left(x_{0}, 1\right], \varphi(x)$ increases in a right neighbourhood of $x_{0}$ and hence, $\kappa_{h}[u](x)>0$ also in a right neighbourhood of $x_{0}$.

In view of the strong minimum principle of (2.5) considered as a second order equation for $\kappa_{h}[u]$ and applied to a possible minimum 0 , is suffices to show that $\kappa_{h}[u] \geq 0$ everywhere. We assume by contradiction that there exist $x_{0}<x^{\prime}<x^{\prime \prime}<1$ such that $\kappa_{h}[u]>0$ (i.e. $\varphi^{\prime}>0$ ) in $\left[0, x^{\prime}\right)$ and $\kappa_{h}[u]<0$ (i.e. $\left.\varphi^{\prime}<0\right)$ in $\left(x^{\prime}, x^{\prime \prime}\right)$. In particular, $u^{\prime \prime}<0$ in $\left[x^{\prime}, x^{\prime \prime}\right)$ and so, also on a slightly larger interval $\left(x^{*}, x^{\prime \prime}\right) \supset\left[x^{\prime}, x^{\prime \prime}\right)$. Finally, we may find $x_{1} \in\left(x^{*}, x^{\prime}\right)$ and $x_{2} \in\left(x^{\prime}, x^{\prime \prime}\right)$ with $\varphi\left(x_{1}\right)=\varphi\left(x_{2}\right)$.

Then, with the same notation as in the proof of Theorem 6.4, we consider the function $v \in$ $C^{1,1}([-\ell(\varrho), \ell(\varrho)],(0, \infty))$ defined as in (6.4). Notice that in this case $\varrho<1$. Indeed, by our assumption on $\varphi$ and since $u^{\prime}(x)>0$ in $\left(x_{0}, 1\right]$, the integrand in (6.5) is strictly positive in $\left[x_{1}, x^{\prime}\right)$ and negative in $\left(x^{\prime}, x_{2}\right]$. Moreover since $u^{\prime}$ is strictly decreasing in $\left[x_{1}, x_{2}\right]$, also $\frac{u^{\prime}}{\sqrt{1+u^{\prime 2}}}$ is monotonically decreasing. Splitting the integral in (6.5) and using that $\varphi\left(x_{1}\right)=\varphi\left(x_{2}\right)$ we get

$$
r\left(x_{2}\right)>r\left(x_{1}\right)+\frac{u^{\prime}\left(x^{\prime}\right)}{\sqrt{1+u^{\prime}\left(x^{\prime}\right)^{2}}} \int_{x_{1}}^{x^{\prime}} \varphi^{\prime}(t) d t+\frac{u^{\prime}\left(x^{\prime}\right)}{\sqrt{1+u^{\prime}\left(x^{\prime}\right)^{2}}} \int_{x^{\prime}}^{x_{2}} \varphi^{\prime}(t) d t=r\left(x_{1}\right) .
$$

Then, $w(x):=\frac{1}{\ell(\varrho)} v(\ell(\varrho) x), x \in[-1,1]$, satisfies

$$
w^{\prime}(1)=-\beta, \quad w(1)=\frac{\varrho \alpha}{\ell(\varrho)} \quad \text { and } \quad \mathcal{W}_{h}(w)<\mathcal{W}_{h}(u)
$$

Since $\varphi>0$ on $(0,1]$ we have $\varrho<\ell(\varrho)$ and $w(1)<u(1)$. Therefore, $\mathcal{W}_{h}(w)>\mathcal{W}_{h}(u)$ by monotonicity of the energy (see Propositions 4.40 and 4.49), which contradicts the inequality in (6.9).

\section{Numerical studies and algorithms}

We will try to approximate a solution $u(x)$ of the Willmore equation (1.4) by approximating the stationary limit $\widetilde{u}(x)=\lim _{t \rightarrow \infty} U(x, t)$ of the solution $U(x, t)$ of the Dirichlet problem of the 
Willmore flow equation

$$
\left\{\begin{array}{l}
V=\triangle_{\Gamma} H+2 H^{3}-2 H K \quad \forall x \in(-1,1), t>0, \\
U(-1, t)=U(1, t)=\alpha, \quad U_{x}(-1, t)=-U_{x}(1, t)=\beta \quad \forall t>0 \\
U(x, 0)=u_{0}(x) \quad \forall x \in[-1,1]
\end{array}\right.
$$

for a family $(\Gamma(t))_{t \in[0, \infty)}$ of axially symmetric surfaces parametrised by

$$
\Gamma(t):=\{(x, U(x, t) \cos \varphi, U(x, t) \sin \varphi): x \in[-1,1], \varphi \in[0,2 \pi]\} .
$$

Here $H, K$ denote the quantities related to the surface $\Gamma(t)$ generated by the function $U(x, t)$ and $V$ the normal velocity of $\Gamma(t)$ given by

$$
V=\frac{U_{t}}{\left(1+U_{x}^{2}\right)^{1 / 2}}
$$

In order to derive the variational formulation of (7.10) we exploit the fact that the right hand side of (7.10) is linked with the derivative of the Willmore functional

$$
\mathcal{W}(\Gamma)=\int_{\Gamma} H^{2} d A
$$

In fact, writing $\mathcal{W}(U)$ instead of $\mathcal{W}(\Gamma)$, we can show that

$$
\mathcal{W}(U)=2 \pi \widetilde{\mathcal{W}}(U)+2 \pi \frac{\beta}{\sqrt{1+\beta^{2}}}
$$

where

$$
\widetilde{\mathcal{W}}(U):=\frac{1}{4} \int_{-1}^{+1}\left\{\frac{U U_{x x}^{2}}{\left(1+U_{x}^{2}\right)^{5 / 2}}+\frac{1}{U\left(1+U_{x}^{2}\right)^{1 / 2}}\right\} d x
$$

Thus, for the derivative in direction $\phi \in H_{0}^{2}(-1,1)$, we obtain

$$
\begin{aligned}
\left\langle\widetilde{\mathcal{W}}^{\prime}(U), \phi\right\rangle:= & \left.\frac{d}{d \varepsilon} \widetilde{\mathcal{W}}(U+\varepsilon \phi)\right|_{\varepsilon=0} \\
= & \frac{1}{4} \int_{-1}^{+1}\left\{2 \frac{U U_{x x} \phi_{x x}}{\left(1+U_{x}^{2}\right)^{5 / 2}}+\frac{U_{x x}^{2} \phi}{\left(1+U_{x}^{2}\right)^{5 / 2}}-5 \frac{U U_{x} U_{x x}^{2} \phi_{x}}{\left(1+U_{x}^{2}\right)^{7 / 2}}\right. \\
& \left.\quad-\frac{\phi}{U^{2}\left(1+U_{x}^{2}\right)^{1 / 2}}-\frac{U_{x} \phi_{x}}{U\left(1+U_{x}^{2}\right)^{3 / 2}}\right\} d x
\end{aligned}
$$

Under the smoothness assumption $U \in H^{4}(-1,1)$ one can prove (see $\left.\S 2.3\right)$ that

$$
\left\langle\widetilde{\mathcal{W}}^{\prime}(U), \phi\right\rangle=-\int_{-1}^{+1} U \phi\left(\triangle_{\Gamma} H+2 H^{3}-2 H K\right) d x \quad \forall \phi \in H_{0}^{2}(-1,1) .
$$

Multiplying (7.10) with the test function $U \phi$ and integrating over $[-1,1]$ yields the following variational formulation :

For $t \geq 0$ find $U(\cdot, t) \in X$ such that $U(., 0)=u_{0}$ and

$$
\int_{-1}^{+1} \frac{U(\cdot, t) U_{t}(\cdot, t) \phi}{\left(1+U_{x}(\cdot, t)^{2}\right)^{1 / 2}} d x+\left\langle\widetilde{\mathcal{W}}^{\prime}(U(\cdot, t)), \phi\right\rangle=0 \quad \forall \phi \in H_{0}^{2}(-1,1), t>0
$$


where $\left\langle\widetilde{\mathcal{W}}^{\prime}(U(\cdot, t)), \phi\right\rangle$ is defined by $(7.12)$ and

$$
X:=\left\{v \in H^{2}(-1,1): v(-1)=v(1)=\alpha, v^{\prime}(-1)=-v^{\prime}(1)=\beta\right\} .
$$

For the numerical solution of (7.14), we use the finite element method to get a finite dimensional nonlinear system of ODEs. To this end, we decompose the space interval $I=[-1,1]$ into elements $K_{i}=\left[x_{i-1}, x_{i}\right], i=1, \ldots, N$, and define the finite element space $X_{h} \subset X$ as

$$
X_{h}:=\left\{v \in X:\left.v\right|_{K_{i}} \in \mathbb{P}_{3} \quad \forall i=1, \ldots, N\right\},
$$

where $\mathbb{P}_{3}$ denotes the space of polynomials with degree less or equal to 3 . Note that $X_{h} \subset C^{1}(I, \mathbb{R})$, i.e. we use $C^{1}$-elements of third order. The degrees of freedom are the values of the function and of the first derivative at the nodes $x_{i}$ where the values at the boundary points $x_{0}=-1$ and $x_{N}=1$ are prescribed by the values $\alpha$ and $\beta$ due to $X_{h} \subset X$. Thus, the semi-discrete solution $U_{h}(\cdot, t) \in X_{h}$ of problem (7.14) can be represented as

$$
U_{h}(x, t)=\sum_{j=1}^{2 N+2} c_{j}(t) \varphi_{j}(x),
$$

where the basis functions $\varphi_{j} \in C^{1}(I, \mathbb{R})$ are defined as follows. For each element $K_{i}$, it holds $\left.\varphi_{j}\right|_{K_{i}} \in \mathbb{P}_{3}$ for all $j$. The first set of basis functions $\varphi_{1+i}, i=0, \ldots, N$, is responsible for the point values of the discrete function at the nodes $x_{i}$, i.e., it holds

$$
\varphi_{1+i}\left(x_{k}\right)=\delta_{i, k}, \quad \frac{\partial}{\partial x} \varphi_{1+i}\left(x_{k}\right)=0 \quad \forall k=0, \ldots, N, \quad i=0, \ldots N .
$$

The second set $\varphi_{N+2+i}$ is responsible for the values of the $x$-derivatives at the nodes $x_{i}$, i.e., it holds

$$
\varphi_{N+2+i}\left(x_{k}\right)=0, \quad \frac{\partial}{\partial x} \varphi_{N+2+i}\left(x_{k}\right)=\delta_{i, k} \quad \forall k=0, \ldots, N, \quad i=0, \ldots N .
$$

These conditions for the definition of the basis functions $\varphi_{j}$ imply the following meaning of the coefficients $c_{j}(t)$ in $(7.16)$

$$
c_{1+i}(t)=U_{h}\left(x_{i}, t\right), \quad c_{N+2+i}(t)=\frac{\partial}{\partial x} U_{h}\left(x_{i}, t\right) \quad \forall i=0, \ldots, N,
$$

where $c_{1}(t)=c_{N+1}(t)=\alpha$ and $c_{N+2}(t)=-c_{2 N+2}(t)=\beta$ for all $t>0$ due to the Dirichlet boundary conditions of $U_{h}(x, t)$. Note that the support of the basis functions $\varphi_{1+i}$ and $\varphi_{N+2+i}$ is local; it consists of the (at most two) elements that contain the node $x_{i}$. The initial condition is discretised as $U_{h}(x, 0)=u_{0, h}(x)=\sum_{j=1}^{2 N+2} c_{0, j} \varphi_{j}(x)$, where $u_{0, h} \in X_{h}$ is a suitable interpolant of $u_{0}$ in $X_{h}$, which can be defined, for instance, by the choice $c_{0,1+i}:=u_{0}\left(x_{i}\right)$ and $c_{0, N+2+i}:=\frac{\partial}{\partial x} u_{0}\left(x_{i}\right)$ for $i=0, \ldots, N$. Therefore, we have the initial conditions $c_{j}(0)=c_{0, j}$ for the unknown coefficient functions $c_{j}(t)$. For the discrete problem, we need the test space

$$
X_{h, 0}:=\left\{v \in H_{0}^{2}(-1,1):\left.v\right|_{K_{i}} \in \mathbb{P}_{3} \quad \forall i=1, \ldots, N\right\} .
$$

Then, the semi-discrete variational problem reads :

For $t \geq 0$ find $U_{h}(\cdot, t) \in X_{h}$ such that $U_{h}(., 0)=u_{0, h}$ and

$$
\int_{-1}^{+1} \frac{U_{h}(\cdot, t) U_{h t}(\cdot, t) \phi_{h}}{\left(1+U_{h x}(\cdot, t)^{2}\right)^{1 / 2}} d x+\left\langle\widetilde{\mathcal{W}}^{\prime}\left(U_{h}(\cdot, t)\right), \phi_{h}\right\rangle=0 \quad \forall \phi_{h} \in X_{h, 0}, t>0 .
$$


Using the ansatz (7.16) for $U_{h}(\cdot, t)$ and taking the test functions $\phi_{h}=\varphi_{i} \in X_{h, 0}$ for $i \in J_{h}:=$ $\{1, \ldots, 2 N+2\} \backslash\{1, N+1, N+2,2 N+2\}$, we see that (7.17) is equivalent to a nonlinear $(2 N-2)$ dimensional system of ODEs for the coefficient functions $c_{j}(t), j \in J_{h}$.

In the time discretisation, we calculate for a discrete time level $t_{n}$ an approximation $U^{n} \in X_{h}$ of $U_{h}\left(\cdot, t_{n}\right)$. Starting with $t_{0}=0$ and $U^{0}:=u_{0, h}$, we assume that $U^{n}$ is known. We choose a time step $k_{n}>0$ and compute $U^{n+1}$ at the time level $t_{n+1}:=t_{n}+k_{n}$ from (7.17) by approximating the time derivative $U_{h t}$ at $t=t_{n}$ by the first order backward difference formula

$$
U_{h t}\left(t_{n}\right) \approx \frac{U^{n+1}-U^{n}}{k_{n}}
$$

In order to get a linear system of equations for $U^{n+1}$ we replace in (7.17) several nonlinear terms of $U_{h}(\cdot, t)$ by the known function $U^{n} \in X_{h}$, i.e., we compute $U^{n+1} \in X_{h}$ from

$$
\int_{-1}^{+1} \frac{U^{n}}{\left(1+\left(U_{x}^{n}\right)^{2}\right)^{1 / 2}}\left(U^{n+1}-U^{n}\right) \phi_{h} d x+k_{n}\left\langle\widetilde{\mathcal{W}}_{n}^{\prime}\left(U^{n+1}\right), \phi_{h}\right\rangle=0 \quad \forall \phi_{h} \in S_{h},
$$

where $\widetilde{\mathcal{W}}_{n}^{\prime}\left(U^{n+1}\right)$ is the following linear approximation of $\widetilde{\mathcal{W}}^{\prime}\left(U^{n+1}\right)$ :

$$
\begin{aligned}
\left\langle\widetilde{\mathcal{W}}_{n}^{\prime}\left(U^{n+1}\right), \phi_{h}\right\rangle:=\frac{1}{4} \int_{-1}^{+1} & \left\{2 \frac{U^{n} U_{x x}^{n+1} \phi_{h x x}}{\left(1+\left(U_{x}^{n}\right)^{2}\right)^{5 / 2}}+\frac{\left(U_{x x}^{n}\right)^{2} \phi_{h}}{\left(1+\left(U_{x}^{n}\right)^{2}\right)^{5 / 2}}-5 \frac{U^{n}\left(U_{x x}^{n}\right)^{2} U_{x}^{n+1} \phi_{h x}}{\left(1+\left(U_{x}^{n}\right)^{2}\right)^{7 / 2}}\right. \\
& \left.-\frac{\phi_{h}}{\left(U^{n}\right)^{2}\left(1+\left(U_{x}^{n}\right)^{2}\right)^{1 / 2}}-\frac{U_{x}^{n+1} \phi_{h x}}{U^{n}\left(1+\left(U_{x}^{n}\right)^{2}\right)^{3 / 2}}\right\} d x .
\end{aligned}
$$

Note that the places, where we have taken $U^{n}$ and where $U^{n+1}$, have been chosen heuristically. Other choices are possible and will be studied in future.

Numerical experiments have shown that the choice of a constant time step $k_{n}=\Delta t$ for all $n=0,1, \ldots$, does not lead to satisfying results. If the time step is too large the sequence $\left\{U^{n}\right\}$ can be divergent and if it is too small one needs a very large computing time to reach a stationary limit. Therefore, we have developed an adaptive time step control which is presented in Figure 14. In the step (b2) we discard the computed solution $U^{n+1}$ if its energy has increased compared with $U^{n}$ or if the relative change of the energy was too large. We divide the time step size by two and compute $U^{n+1}$ again. In all other cases we accept the solution $U^{n+1}$. Moreover, we double the size for the next time step if the relative change of the energy was at least in two previous time steps too small and the doubled size is not larger than a prescribed value $k_{\max }$.

In our numerical experiments, we have chosen the control parameters $\omega_{\max }=0.1, \omega_{\min }=0.01$, $k_{\max }=0.01$ (see Figure 14) and the initial time step size $k_{0}=h^{4}$ where $h:=\max _{1 \leq i \leq N}\left|x_{i}-x_{i-1}\right|$ denotes the mesh-size. We accept $U^{n+1}$ to be the stationary limit of our time marching algorithm if

$$
k_{n}^{-1}\left|\mathcal{W}\left(U^{n+1}\right)-\mathcal{W}\left(U^{n}\right)\right|<\varepsilon_{\mathcal{W}} \quad \text { and } \quad k_{n}^{-1}\left\|U^{n+1}-U^{n}\right\|_{\infty}<\varepsilon_{U},
$$

where the norm $\|\cdot\|_{\infty}$ is defined as

$$
\left\|U_{h}\right\|_{\infty}:=\max _{1 \leq j \leq 2 N+2}\left|c_{j}\right| \quad \text { for } \quad U_{h}=\sum_{j=1}^{2 N+2} c_{j} \varphi_{j} \in X_{h},
$$

and $\varepsilon_{\mathcal{W}}, \varepsilon_{U}$ are some prescribed tolerances which have been chosen as $\varepsilon_{\mathcal{W}}=\varepsilon_{U}=10^{-4}$. We stop the time iteration if the criterion (7.19) is satisfied or if, in case (b2) of the adaptive time step control (Figure 14), for the halved time step size it holds $k_{n} / 2<\sqrt{e p s}$, where eps is the relative floating-point accuracy, or if a maximum number $n_{\max }$ of iteration steps is reached (we have used $\left.n_{\max }=360000\right)$. 
Computation of $\left(t_{n+1}, k_{n+1}, U^{n+1}\right)$ from $\left(t_{n}, k_{n}, U^{n}\right)$ :

(a) initialize: $\mathcal{W}_{0}:=\mathcal{W}\left(U^{n}\right) \quad$ formula $\left.(7.11)\right) ; T^{\text {accept }}:=0 ; N^{\text {too_small }}:=0 ;$

(b) while $T^{a c c e p t}=0$ do

(1) compute: $U^{n+1}$ by solving $(7.18) ; \mathcal{W}:=\mathcal{W}\left(U^{n+1}\right)$;

(2) if $\mathcal{W}>\mathcal{W}_{0}$ or $\quad\left|\mathcal{W}-\mathcal{W}_{0}\right|>\omega_{\max }\left|\mathcal{W}_{0}\right|$ then $k_{n}:=k_{n} / 2$;

(3) else if $\left|\mathcal{W}-\mathcal{W}_{0}\right|<\omega_{\min }\left|\mathcal{W}_{0}\right|$ and $2 k_{n}<k_{\max }$ then $T^{\text {accept }}:=1$;

if $\quad N^{\text {too_small }} \geq 2$ then

$k_{n}:=2 k_{n} ; \quad N^{\text {too_small }}:=0$

else

$N^{\text {too_small }}:=N^{\text {too_small }}+1$

endif

(4) else

$T^{\text {accept }}:=1$

endif

enddo

(c) $k_{n+1}:=k_{n} ; \quad t_{n+1}:=t_{n}+k_{n} ;$

Figure 14: Time marching algorithm with control parameters $\omega_{\max }, \omega_{\min }$ and $k_{\max }$.

In the following, we describe our numerical results for three different settings of the boundary data $\alpha$ and $\beta$. In the first case, we combine the value $\alpha=0.5$ with the three negative values $\beta \in\{-1,-5,-10\}$, see Figure 15. In the second case, we consider $\alpha=0.5$ and the positive values $\beta \in\{1,5,10\}$, see Figure 16. Finally, in the third case, we study the situations where $\beta=0$ and $\alpha \in\{0.5,0.1,0.01\}$, see Figure 17. The finite element mesh depends on the data $\alpha$ and $\beta$ and is locally adapted near the boundary in the following way. The interval $I=[-1,1]$ is decomposed into the three subintervals $\Omega_{1}=[-1,-1+\delta], \Omega_{2}=[-1+\delta, 1-\delta]$ and $\Omega_{3}=[1-\delta, 1]$ where

$$
\delta:=\min \left\{\frac{2}{3}, \omega_{0} \min \left\{|\alpha|, \frac{1}{|\beta|+10^{-6}}\right\}\right\}
$$

with $\omega_{0}=2$ except for the case $(\alpha, \beta)=(0.01,0)$ where $\omega_{0}=8$. Then the mesh is created by subdividing each subinterval $\Omega_{k}$ into $N_{0}=40$ equidistant elements. In each picture of Figures 15 17 , the mesh is shown on the $x$-axis, the initial solution $U^{0}$ at $t_{0}=0$ is presented by the dotted line and the final solution $U^{n}$ at the end $t_{n}$ of the time iteration is given by the solid line. The parameters at the headline of the picture have the following meaning. $\mathrm{n}$ denotes the number of the last time step, dt the last time step size $k_{n}$, NEL the number of the finite elements and $\mathrm{W}$ the value of the Willmore functional $\mathcal{W}\left(U^{n}\right)$.

For nearly all cases, our numerical algorithm produced a discrete solution satisfying the approximate "stationarity" criterion (7.19) with the tolerances $\varepsilon_{\mathcal{W}}=\varepsilon_{U}=10^{-4}$. The first exceptional case was $(\alpha, \beta)=(0.5,-10)$ where the algorithm at a time $t_{n}$ could not find a next function $U^{n+1}$ at a time $t_{n}+k_{n}$ with $k_{n}>\sqrt{e p s}$ such that $\mathcal{W}\left(U^{n+1}\right)<\mathcal{W}\left(U^{n}\right)$. The other critical case was $(\alpha, \beta)=(0.01,0)$ where for all $n$ a new $U^{n+1}$ with a smaller value of the Willmore functional 


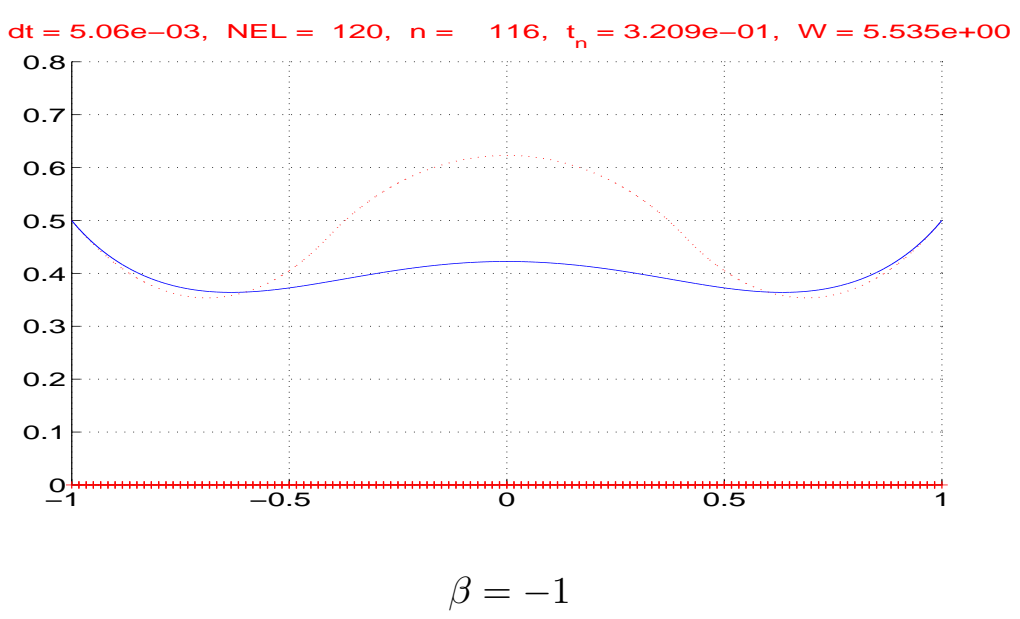

$\mathrm{dt}=5.24 \mathrm{e}-\mathrm{O} 3, \mathrm{NEL}=120, \mathrm{n}=262, \mathrm{t}_{\mathrm{n}}=7.671 \mathrm{e}-01, \mathrm{~W}=8.523 \mathrm{e}+\mathrm{O} 0$

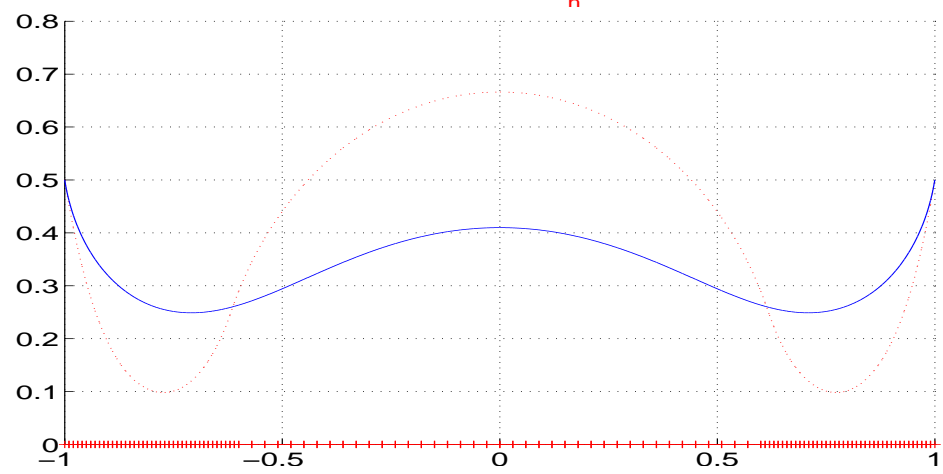

$\beta=-5$

$\mathrm{dt}=2.00 \mathrm{e}-08, \mathrm{NEL}=120, \mathrm{n}=2864, \mathrm{t}_{\mathrm{n}}=4.683 \mathrm{e}-01, \mathrm{~W}=9.258 \mathrm{e}+\mathrm{O} 0$

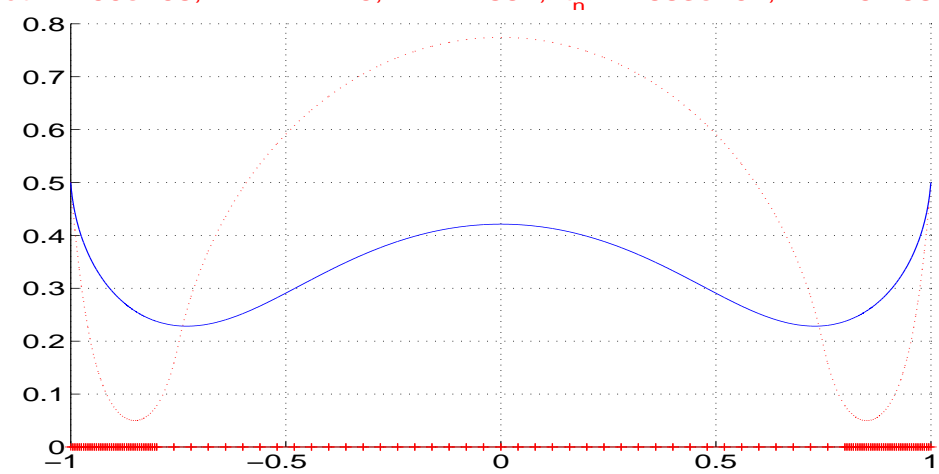

$\beta=-10: \quad k_{n}^{-1}\left\|U^{n+1}-U^{n}\right\|_{\infty} \approx 1.67 e-02, \quad$ stopped by $k_{n} / 2<\sqrt{e p s}$

Figure 15: $\alpha=0.5$ and $\beta<0$ 

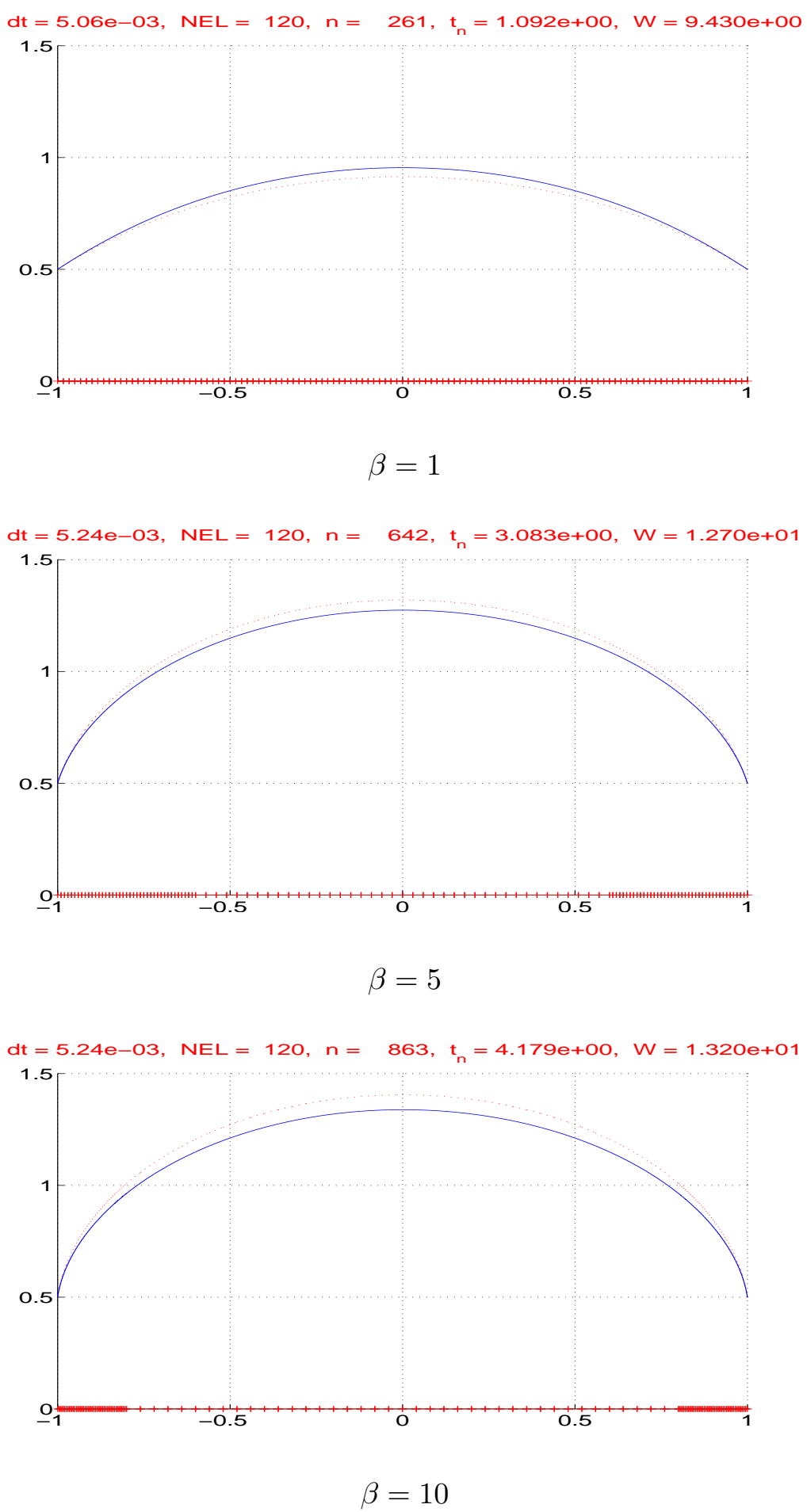

Figure 16: $\alpha=0.5$ and $\beta>0$ 


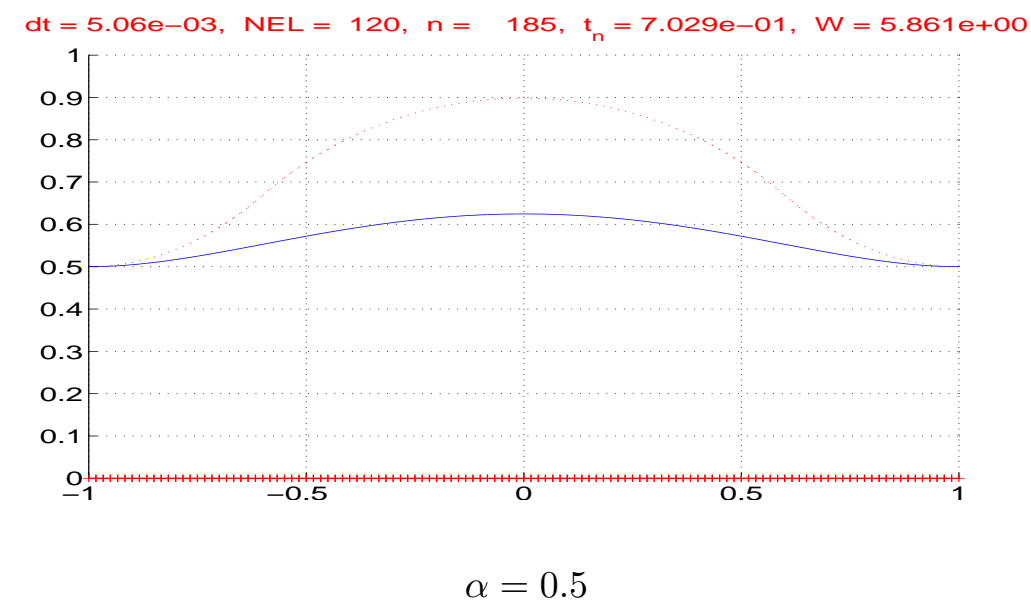

$\mathrm{dt}=5.24 \mathrm{e}-\mathrm{O} 3, \mathrm{NEL}=120, \mathrm{n}=372, \mathrm{t}_{\mathrm{n}}=1.604 \mathrm{e}+\mathrm{OO}, \mathrm{W}=1.113 \mathrm{e}+\mathrm{O} 1$

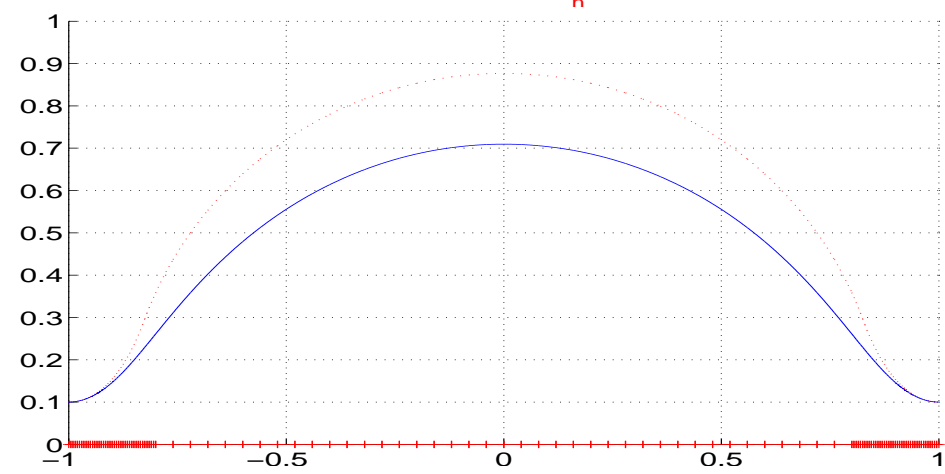

$$
\alpha=0.1
$$

$\mathrm{dt}=5.24 \mathrm{e}-\mathrm{O} 7, \mathrm{NEL}=120, \mathrm{n}=360000, \mathrm{t}_{\mathrm{n}}=1.447 \mathrm{e}-01, \mathrm{~W}=1.244 \mathrm{e}+\mathrm{O} 1$

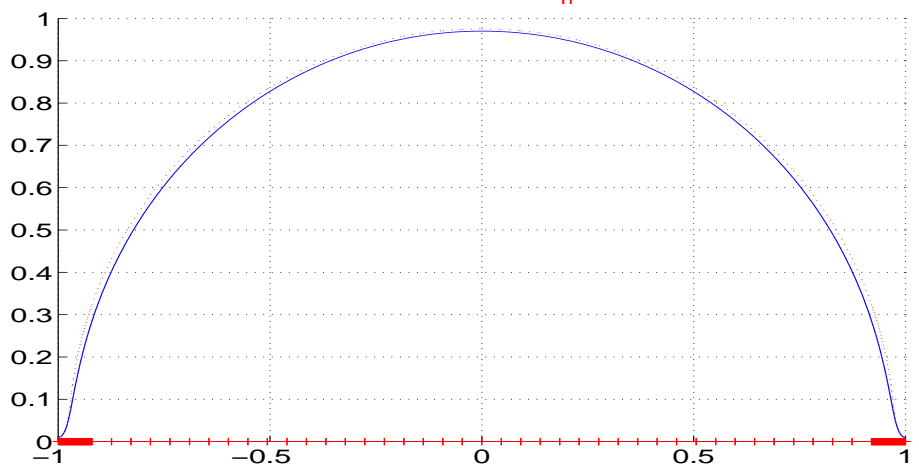

$\alpha=0.01: \quad$ stopped by $n=n_{\max }, \quad\left\|U^{n+1}-U^{n}\right\|_{\infty} \approx 2.75 e-04$

Figure 17: $\beta=0$ 
could be found but where the maximum number of $n_{\max }=360000$ time steps was reached without satisfying the criterion (7.19). On the other hand, the change of the graph of the discrete solution as well as the time step size over the last $99 \%$ of all time steps was very small. Here, further research is necessary to figure out the reason for this behaviour. Could it be that the first order time discretisation is not accurate enough or that the semi-implicit backward Euler exhibits some instabilities which lead to very small time steps? Another reason could be that the "exact" continuous Willmore flow is really creeping very slowly to the stationary limit. Let us finally note that, for such critical cases, a suitable choice of the initial function $U^{0}=u_{0, h}$ is important for the convergence of the numerical solution to the stationary limit. Here a good analytical feeling is very helpful. We construct $u_{0, h} \in X_{h}$ as the interpolant of a suitable function $u_{0} \in X$ as described above. In the case $\beta \leq 0$, we use $u_{0}=f_{\alpha}$ with $f_{\alpha}$ defined in (5.1) which is a catenoid at the boundary fitted with an arc of a circle centered at the origin. For $\beta>0$, we choose $u_{0}=w$ with $w$ defined in (6.1) which is an arc of a circle centered at $(0, \alpha-1 / \beta)$.

Acknowledgment. The third author is grateful to G. Huisken (Max-Planck-Institute Golm) for suggesting to study boundary value problems for the Willmore equation, to E. Kuwert for pointing out the importance of Reference [LS2] concerning Willmore surfaces of revolution. We thank M. Bergner, K. Deckelnick and R. Schätzle for interesting discussions.

\section{References}

[BK] M. Bauer, E. Kuwert, Existence of minimizing Willmore surfaces of prescribed genus. Int. Math. Res. Not. 2003, no. 10, 553-576, 2003.

[Br] R. Bryant, A duality theorem for Willmore surfaces. J. Differ. Geom. 20, 23-53, 1984.

[BG] R. Bryant, P. Griffiths, Reduction for constrained variational problems and $\int \frac{1}{2} k^{2} d s$. Amer. J. Math. 108, 525-570, 1986.

[DDG] A. Dall'Acqua, K. Deckelnick, H.-Ch. Grunau, Classical solutions to the Dirichlet problem for Willmore surfaces of revolution. Adv. Calc. Var. 1, 379-397, 2008.

[DD] K. Deckelnick, G. Dziuk, Error analysis of a finite element method for the Willmore flow of graphs. Interfaces Free Bound. 8, 21-46, 2006.

[DG1] K. Deckelnick, H.-Ch. Grunau, Boundary value problems for the one-dimensional Willmore equation. Calc. Var. Partial Differential Equations 30, 293-314, 2007.

[DG2] K. Deckelnick, H.-Ch. Grunau, Stability and symmetry in the Navier problem for the one-dimensional Willmore equation. SIAM J. Math. Anal., to appear.

[DG3] K. Deckelnick, H.-Ch. Grunau, A Navier boundary value problem for two-dimensional Willmore surfaces of revolution, in preparation.

[DHKW] U. Dierkes, S. Hildebrandt, A. Küster, O. Wohlrab, Minimal Surfaces. I. Boundary value problems. Grundlehren der mathematischen Wissenschaften 295, SpringerVerlag: Berlin etc., 1992.

[DR] M. Droske, M. Rumpf, A level set formulation for Willmore flow. Interfaces Free Bound. 6, 361-378, 2004.

[Dz] G. Dziuk, Computational parametric Willmore flow. Num. Math. 111, 55-80, 2008.

[E] L. Euler, Opera Omnia, Ser. 1, 24, Zürich: Orell Füssli (1952). 
[He] W. Helfrich, Elastic properties of lipid bylayers: theory and possible experiments, $Z$. Naturforsch. C 28, 693-703, 1973.

[HP] S. Hildebrandt, K. Polthier, Constraint-based fairing of surface meshes. Proceedings of the fifth Eurographics Symposium on Geometry Processing, ACM International Conference Proceeding Series, 257, 203-212, 2007.

[KL] B.G. Konopelchenko, G. Landolfi, On rigid string instantons in four dimensions. Phys. Lett. B 459, 522-526, 1999.

[KS1] E. Kuwert, R. Schätzle, Closed surfaces with bounds on their Willmore energy. Preprint, 2008.

[KS2] E. Kuwert, R. Schätzle, Minimizers of the Willmore functional under fixed conformal class. Preprint, 2008.

[LS1] J. Langer, D. Singer, The total squared curvature of closed curves. J. Differ. Geom. 20, 1-22, 1984.

[LS2] J. Langer, D. Singer, Curves in the hyperbolic plane and mean curvature of tori in 3-space. Bull. London Math. Soc. 16, 531-534, 1984.

[LPP] K. Leschke, F. Pedit, U. Pinkall, Willmore tori in the 4-sphere with nontrivial normal bundle. Math. Ann. 332, 381-394, 2005.

[Ni] J.C.C. Nitsche, Boundary value problems for variational integrals involving surface curvatures. Quart. Appl. Math. 51, 363-387, 1993.

[P] S.D. Poisson, Mémoire sur les surfaces élastiques. Cl. Sci. Mathém. Phys. Inst. de France, 2nd printing, 167-225, 1812.

[R] T. Rivière, Analysis aspects of Willmore surfaces. Invent. Math. 174, 1-45, 2008.

[Sch] R. Schätzle, The Willmore boundary value problem. Preprint, 2006.

[Sn] L. Simon, Existence of surfaces minimizing the Willmore functional. Commun. Anal. Geom. 1, 281-326, 1993.

[Th] G. Thomsen, Über konforme Geometrie I: Grundlagen der konformen Flächentheorie. Abh. Math. Sem. Univ. Hamburg 3, 31-56, 1923.

[Wi] T.J. Willmore, Riemannian geometry. Oxford Science Publications, The Clarendon Press, Oxford University Press, New York, 1993.

[Z] O.-Y. Zhong-can, Elasticity theory of biomembrans. Thin Solid Films 393, 19-23, 2001. 
Anna Dall'Acqua, Fakultät für Mathematik, Otto-von-Guericke-Universität, Postfach 4120, D39016 Magdeburg, Germany

E-mail: anna.dallacqua@ovgu.de

Steffen Fröhlich, Institut für Mathematik, Fachbereich Mathematik und Informatik, Freie Universität Berlin, Arnimallee 3, D-14195 Berlin, Germany

E-mail: sfroehli@math.fu-berlin.de

Hans-Christoph Grunau, Fakultät für Mathematik, Otto-von-Guericke-Universität, Postfach 4120, D-39016 Magdeburg, Germany

E-mail: hans-christoph.grunau@ovgu.de

Friedhelm Schieweck, Fakultät für Mathematik, Otto-von-Guericke-Universität, Postfach 4120, D39016 Magdeburg, Germany

E-mail: friedhelm.schieweck@ovgu.de 